\title{
Examining participant perceptions of benefits, barriers and social support in a diabetes lifestyle program
}

\author{
Samantha Shawley-Brzoska
}

Follow this and additional works at: https://researchrepository.wvu.edu/etd

\section{Recommended Citation}

Shawley-Brzoska, Samantha, "Examining participant perceptions of benefits, barriers and social support in a diabetes lifestyle program" (2018). Graduate Theses, Dissertations, and Problem Reports. 7252. https://researchrepository.wvu.edu/etd/7252

This Dissertation is protected by copyright and/or related rights. It has been brought to you by the The Research Repository @ WVU with permission from the rights-holder(s). You are free to use this Dissertation in any way that is permitted by the copyright and related rights legislation that applies to your use. For other uses you must obtain permission from the rights-holder(s) directly, unless additional rights are indicated by a Creative Commons license in the record and/ or on the work itself. This Dissertation has been accepted for inclusion in WVU Graduate Theses, Dissertations, and Problem Reports collection by an authorized administrator of The Research Repository @ WVU. For more information, please contact researchrepository@mail.wvu.edu. 
Examining participant perceptions of benefits, barriers and social support in a diabetes lifestyle program

Samantha Shawley-Brzoska, MPH

Dissertation Submitted to the West Virginia University

School of Public Health

In Partial Fulfillment of the Requirements for the Degree of

Doctor of Philosophy in

Public Health

Ranjita Misra, PhD, CHES, FASHA, FESG, Chair

Danielle Davidov, PhD

Christa Lilly, PhD

Lesley Cottrell, PhD

Peter Giacobbi, PhD

Department of Social and Behavioral Sciences

$$
\text { Morgantown, West Virginia }
$$

2018

Keywords: Diabetes, Prediabetes, Multiple Methods, Lifestyle Modifications, Benefits, Barriers, Social Support, Prevention, Management

Copyright 2018 Samantha Shawley-Brzoska 


\title{
ABSTRACT \\ Examining participant perceptions of benefits, barriers and social support in a diabetes
}

\author{
lifestyle program
}

\section{by Samantha Shawley-Brzoska}

PURPOSE: The emerging pandemic of chronic diseases is one of the leading causes of morbidity and mortality. West Virginians are disproportionately burdened by diabetes $(15.3 \%)$ and prediabetes (39.5\%); West Virginia (WV) ranks $1^{\text {st }}$ in the nation in the prevalence of diabetes. Evidence-based lifestyle programs can prevent and/or delay the early onset of diabetes and its complications. The Diabetes Prevention Program (DPP) has been shown effectiveness to prevent pre-diabetes in diverse populations and can be implemented in WV communities. It has been administered in faith-based communities that participants improved physical activity, nutrition, and other skills for diabetes prevention. However, these skills also benefit individuals with diabetes. Hence, the evidence-based diabetes prevention and management (DPM) program combined the DPP curriculum with the American Association of Diabetes self-care behaviors (AADE7) for a 12-month lifestyle intervention for individuals with diabetes and prediabetes. The purpose of this dissertation was to use the data from the program to explore participant's perceptions of the benefits, barriers and social support in a community-based program. Program benefits, barriers and social support have been inadequately studied, and an understanding of these non-programmatic measures could provide important information for successful and sustainable lifestyle programs in WV. Currently very few studies have examined these constructs, using qualitative and quantitative analyses techniques in diabetes lifestyle programs. METHODS: Using secondary data analyses, three aims explored (1) participants' perceptions of benefits and barriers of program participation; (2) participants' perceived social support for eating and exercise habits from friends and family (3) in-depth exploration of benefits, barriers and social support for lifestyle changes using focus groups. The first aim included 89 participants (73\% female; 58.51 mean age; $62 \%$ with diabetes) who completed a benefits and barriers survey developed by the investigator. The second aim included 70 participants (74\% female; 59.01 mean age; $61 \%$ with diabetes) who completed social support surveys related to eating and exercise habits. For the third (qualitative) aim, three focus groups were conducted with 22 participants (77\% female; mean age 62.3; 59\% with diabetes). RESULTS: Barriers has a significant relationship with percent body weight while controlling for program attendance $(\mathrm{p}=0.021)$. Lower level of perceived social support for changing dietary habits was associated with better program attendance $(\mathrm{p}=.008)$. Finally, participants revealed they benefited from the program and suggested minimal programmatic changes to improve overall program implementation. CONCLUSION: Quantitative and qualitative approaches were used to triangulate data from program participants and conceptualize how program effectiveness can be achieved in community-based diabetes programs. These results could allow program implementers to tailor future diabetes programs. Identifying the participants' perceptions of benefits, barriers and social support, could be a breakthrough for improving lifestyle programs and their sustainability. Future research on larger, more diverse samples can improve generalizability of the findings for increase in dissemination of diabetes prevention and management education throughout the state of WV and beyond. 


\section{DEDICATION}

To my husband, Derek James Brzoska, who has been my greatest supporter and strongest motivation 


\section{ACKNOWLEDGMENTS}

I write my sincere thanks to my entire Dissertation Committee for taking time and energy out of their busy schedules to support and accommodate my needs throughout the dissertation process. It has been great working with each of them and I have valued their dedication to my success. I was treated like a colleague and given respect as an independent researcher. Dr. Ranjita Misra, you have been my supporter since my earlier days in the Master of Public Health (MPH) program. We have been working together for almost six years and among those years we have implemented three diabetes programs along with a variety of diabetes screenings and presentations. I am grateful for your ongoing support and mentorship over the years, especially the countless hours spent in your office. Your knowledge and compassion for diabetes programming is what made this dissertation possible. Dr. Danielle Davidov, you inspired me from the day I met you and throughout your courses. Your advice and teaching gave a solid foundation, which resulted in the qualitative aim of this dissertation (Chapter 4). This aim could not have completed without your second coding and guidance throughout the process. Dr. Christa Lilly, you have always been amazing to work with and have provided hours of statistical advice with endless patience. Your courses were among the best that I have taken at WVU. Dr. Lesley Cottrell, you have supported me through many endeavors such as teaching and research, but this is by far the longest and most difficult. Your expertise and advice together are very remarkable. Dr. Peter Giacobbi, you have provided support for an extensive amount of time through research and your perspective is always so valuable. This committee is the underlying reason for the completion of this dissertation.

I would also to give thanks to other faculty members within the West Virginia University (WVU) School of Public Health (SPH) and beyond. Dr. Adam Baus and Cecil Pollard, thank you for your flexibility in my full-time work schedule with the Office of Health Services Research (OHSR). The continued support and guidance from the both of you along with the rest of the office is greatly appreciated; we definitely have one of the best. Dr. Keith Zullig, your advice and guidance as a past advisor and Director of the PhD program has been crucial in completing this program. DPM health coaches and volunteers, thank you for your time and effort in helping the program succeed through implementation and data analyses. Dr. Michael Mann and Bobbi Sykes, thank you for always putting your students first and providing support by checking in regularly. Dr. Alfgeir Kristjansson, aim 1 would have not been possible without your class where I developed the benefits and barriers survey. Dr. Jeffrey Coben, you have always provided encouragement and support throughout my public health career. Dr. Gilbert Ramirez, your support while working in our SPH and after leaving has been invaluable and should not go unnoticed. Finally, Dr. Ruth Kershner, your support and persistence was the reason for starting in the field of public health. You are forever missed and will never be replaced in our hearts.

\footnotetext{
"You don't study public health, you live public health."
} 


\section{TABLE OF CONTENTS}

CHAPTER 1 .1

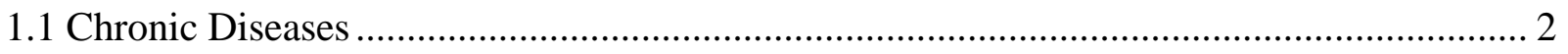

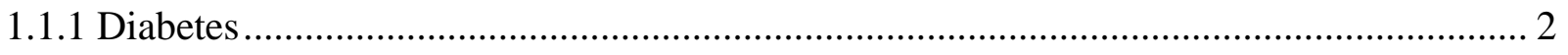

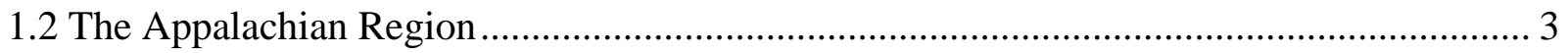

1.3 Diabetes, Risk Factors and Complications .......................................................... 4

1.4 Evidence-Based Lifestyle Programs ................................................................... 4

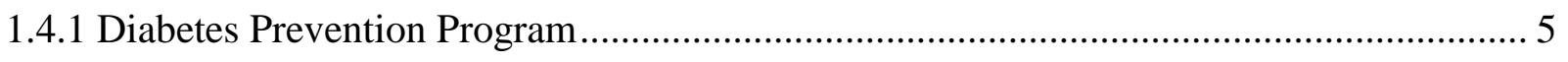

1.4.2 Faith-based Diabetes Prevention Programs..................................................... 6

1.5 Psychosocial Factors in Diabetes Prevention and Management ..................................... 7

1.6 Community-Based Participatory Research .......................................................... 7

1.7 Need for Evidence-based Lifestyle Programs in West Virginia .................................... 8

1.8 Specific Aims and Research Approach ................................................................... 9

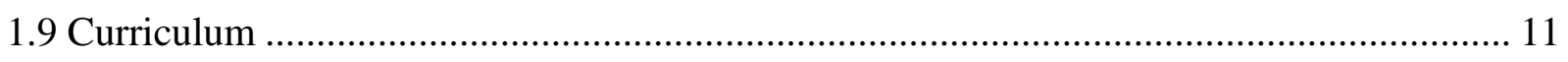

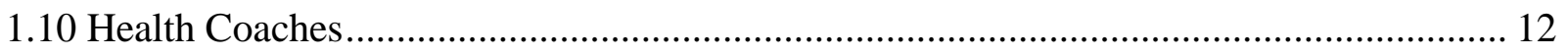

1.11 Study Design and Data Collection Sites ........................................................... 12

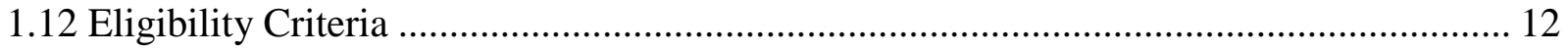

1.13 Participant Screening and Recruitment .............................................................. 13

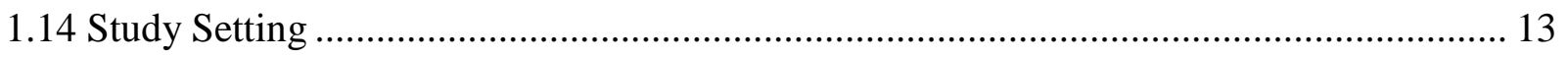

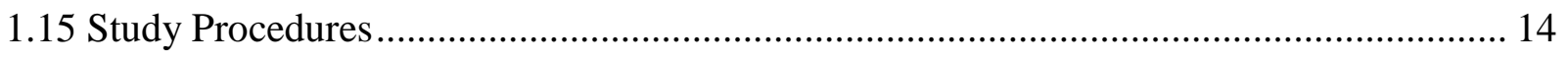

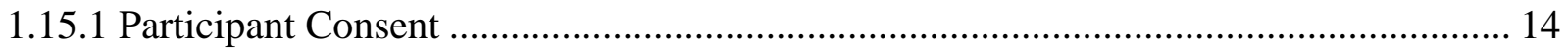

1.15.2 Study Measures and Data Collection .................................................................. 14

1.15.3 Baseline, Mid-Program and Post-Program Assessments ........................................ 14

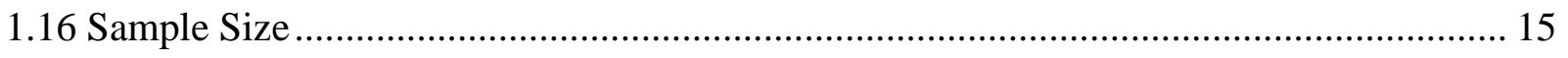

1.17 Participant Barriers and Satisfaction using Focus Groups ......................................... 15

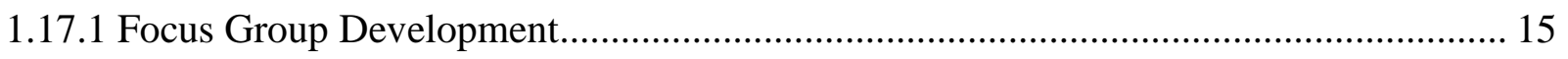

1.17.2 Recruitment and Logistics of Focus Groups ...................................................... 16

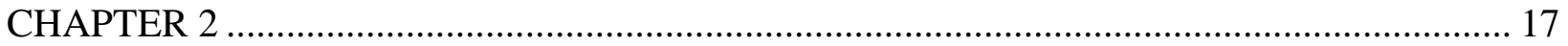

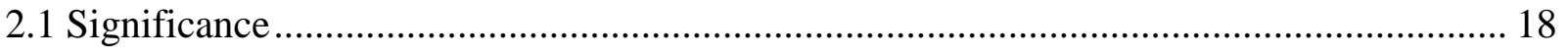

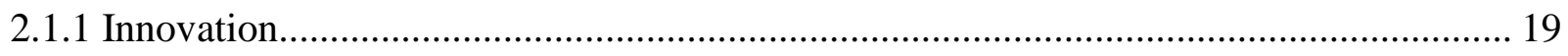




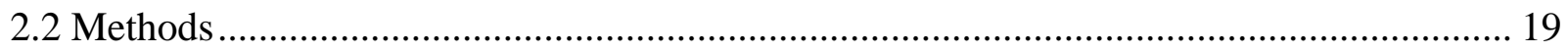

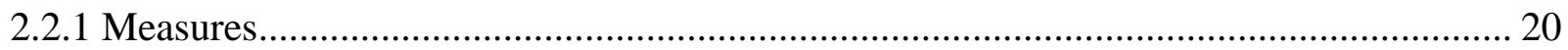

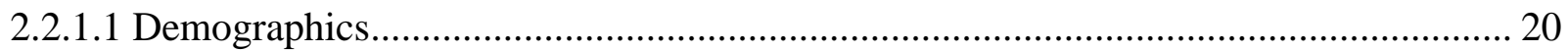

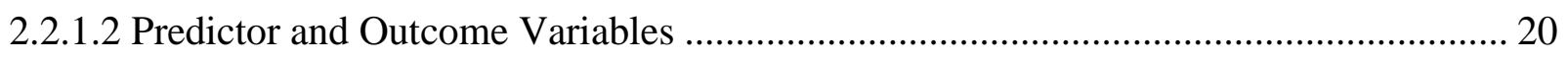

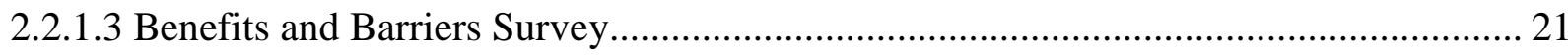

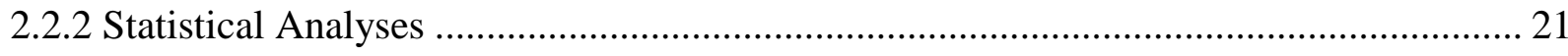

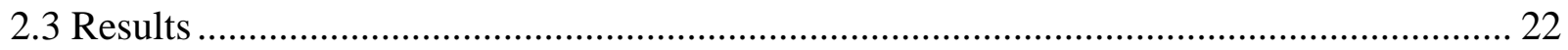

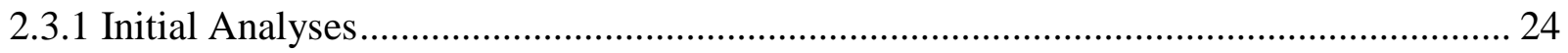

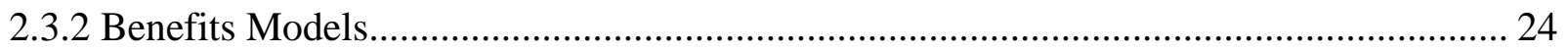

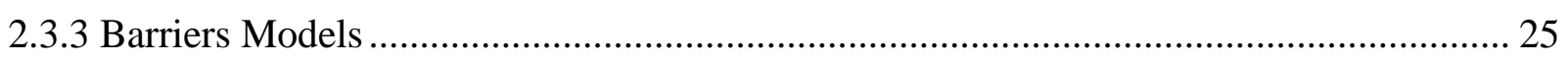

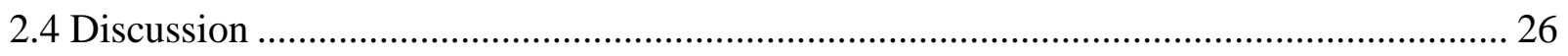

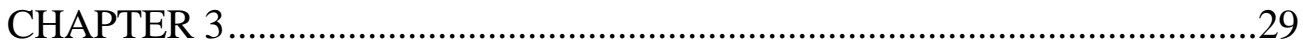

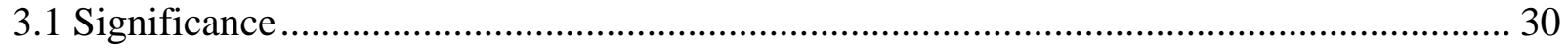

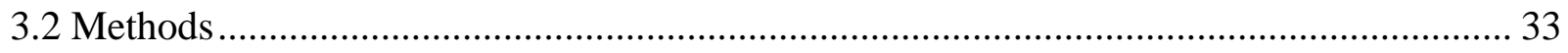

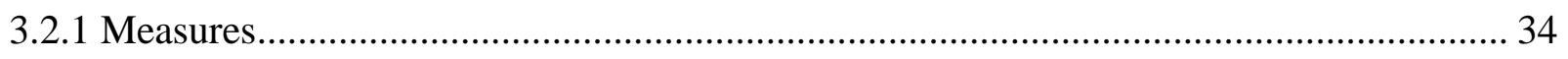

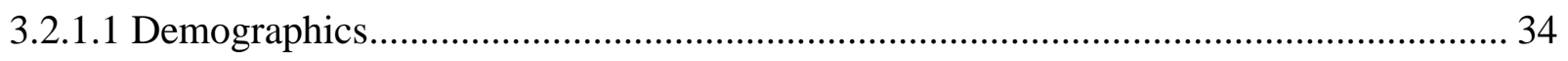

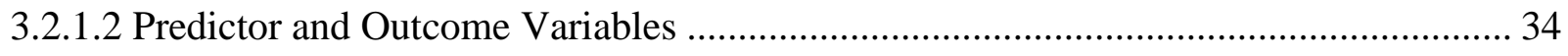

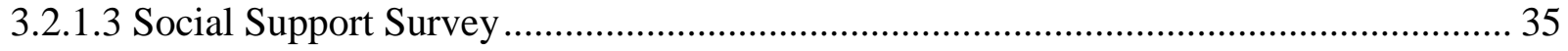

3.2.1.4 Statistical Analyses .............................................................................................. 35

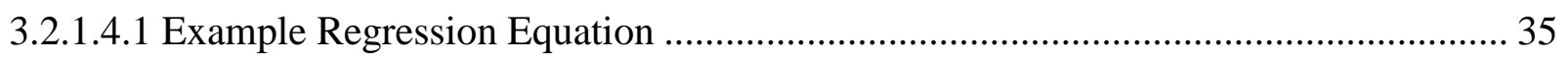

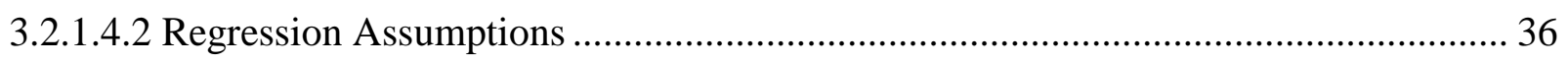

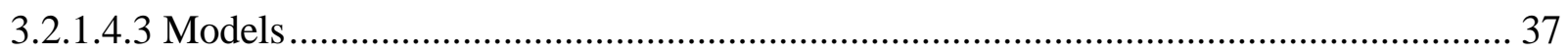

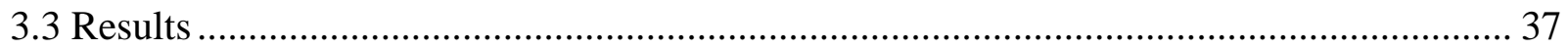

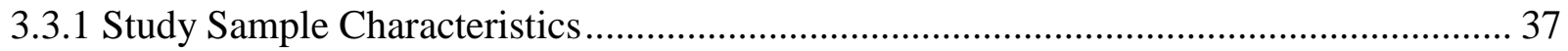

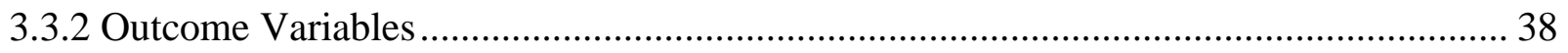

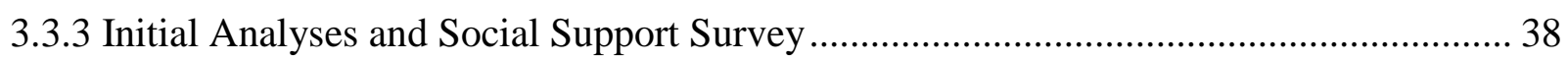

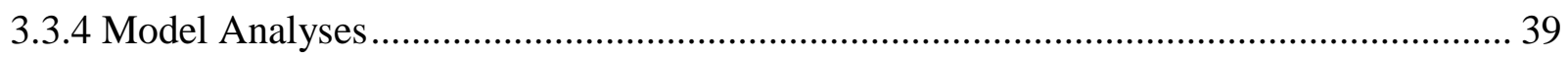

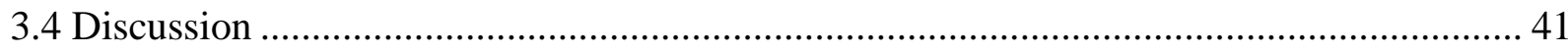

3.5 Conclusion and Implications ....................................................................................... 43

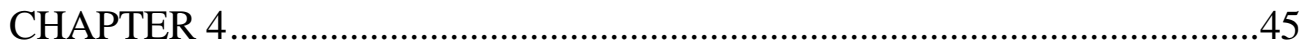

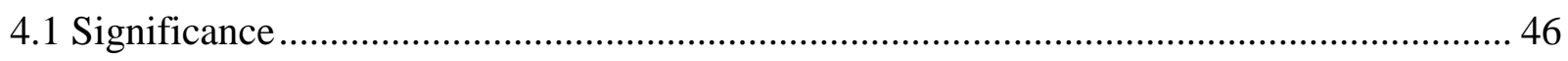

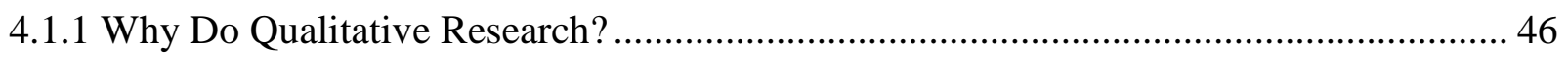

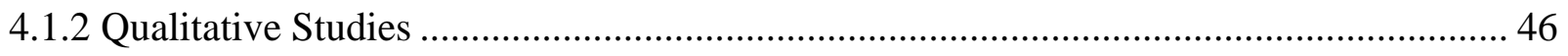




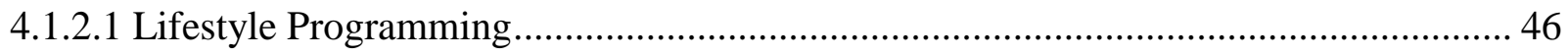

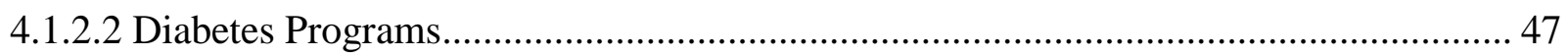

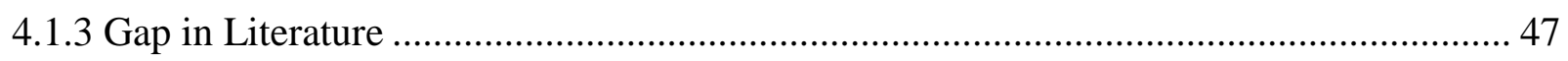

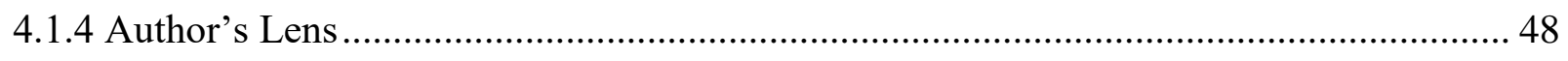

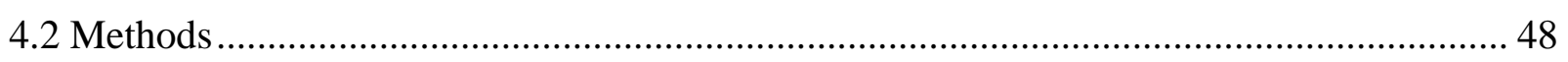

4.2.1 Larger Study Focus Group Development................................................................... 49

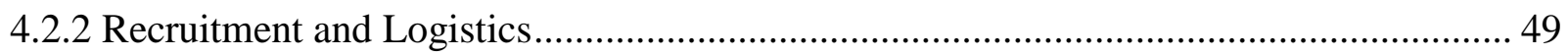

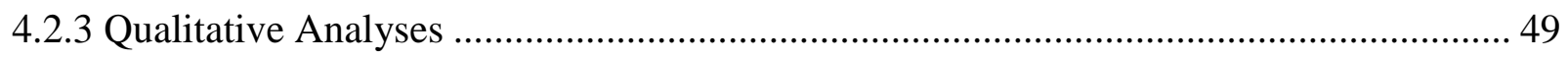

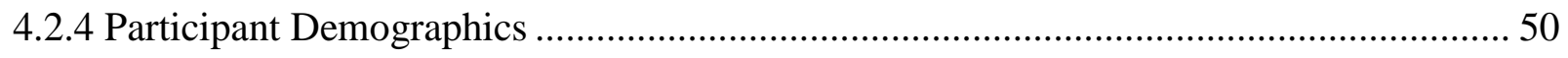

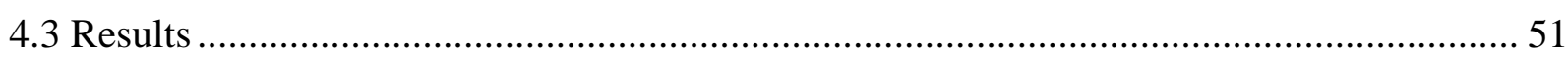

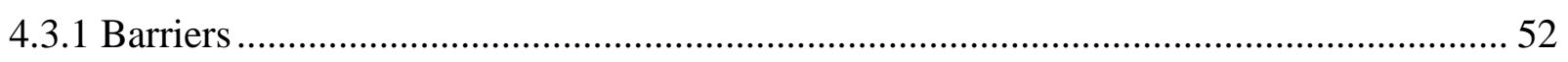

4.3.1.1 Individual Level Health Issues and Behaviors ........................................................ 52

4.3.1.1.1 Eating History and Current Diet Strategies ............................................................. 52

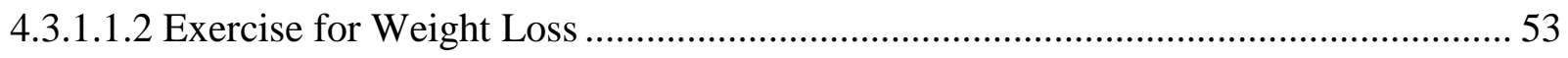

4.3.1.1.3 Chronic and Complex Nature of Diabetes .............................................................. 53

4.3.1.2 Decreased Program Participation ............................................................................. 54

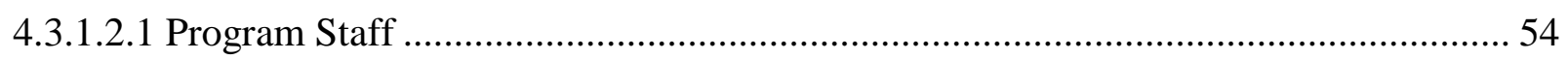

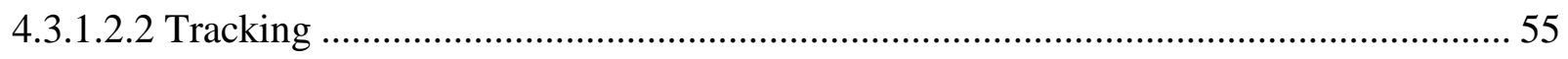

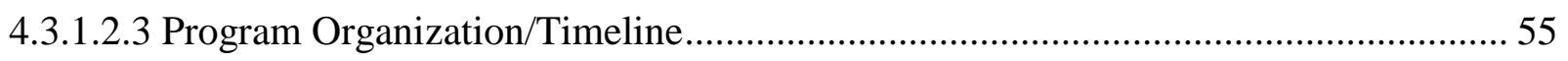

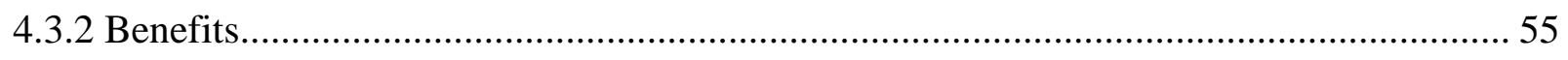

4.3.2.1 Positive Health Behaviors and Social Results from Program ...................................... 56

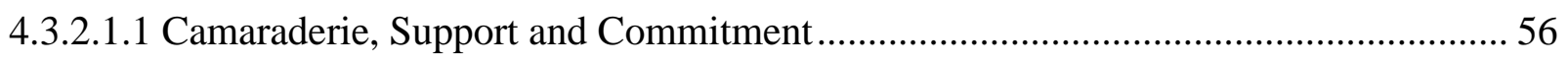

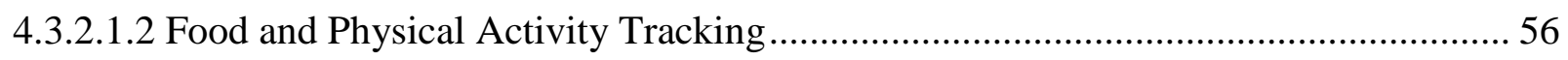

4.3.2.1.3 Health Outcomes and Healthy Behaviors .......................................................... 57

4.3.2.1.4 Knowledge, Acceptance and Control of Diabetes .................................................... 57

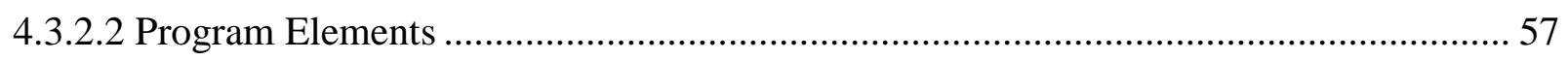

4.3.2.2.1 Health Coaches and Program Leaders ……………............................................. 57

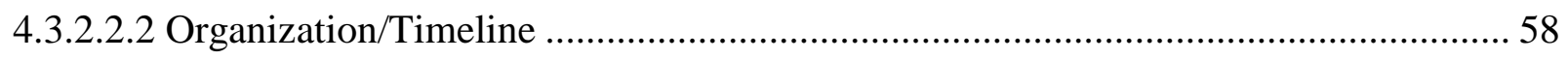

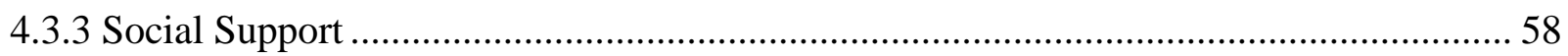

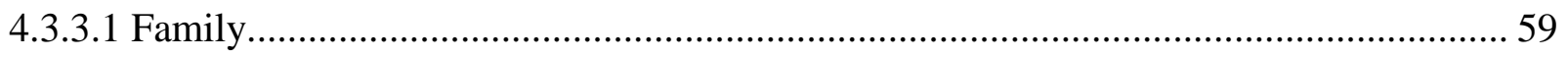

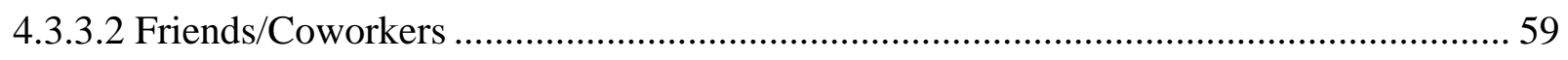

4.3.3.3 Health Coaches/Program Leaders/Fellow Participants .................................................. 60 


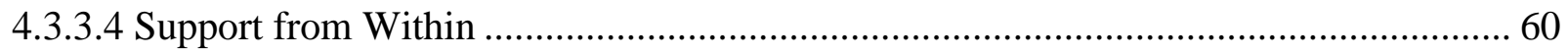

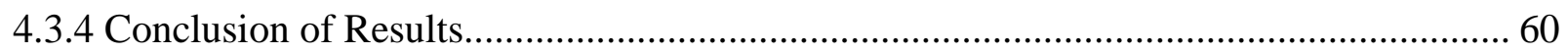

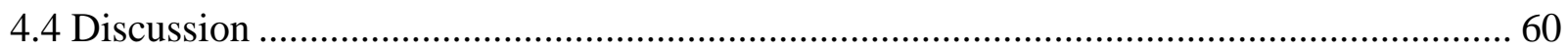

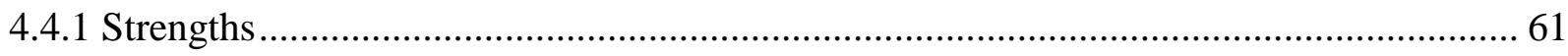

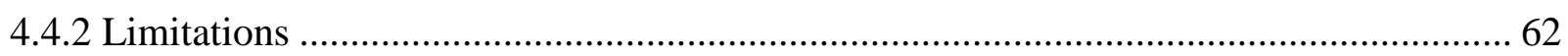

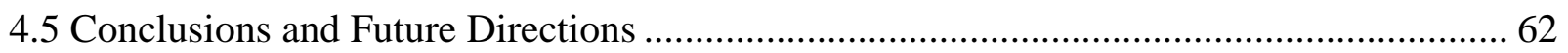

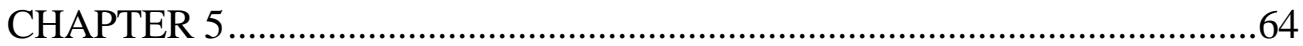

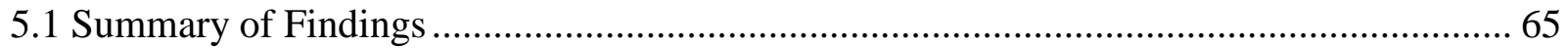

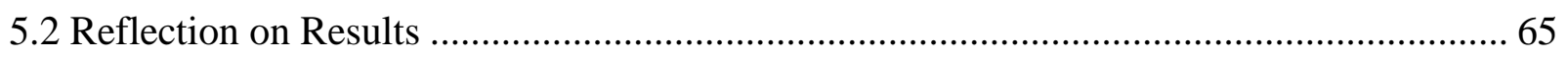

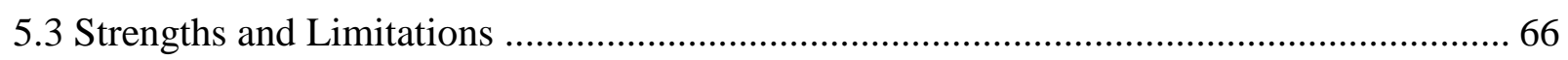

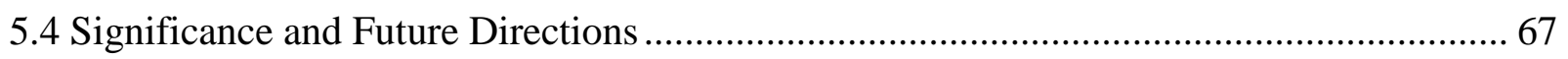

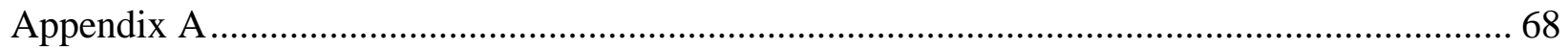

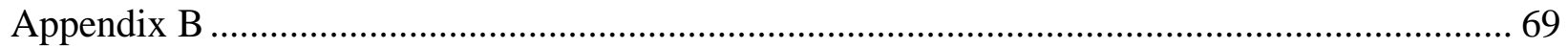

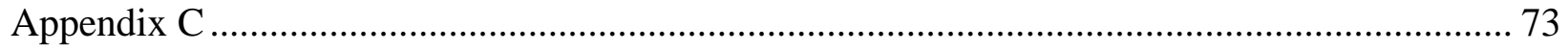

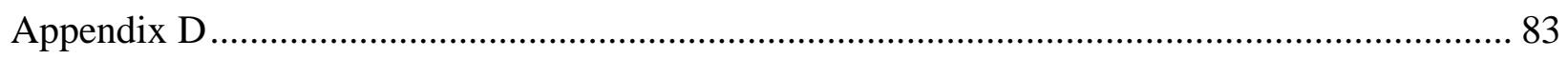

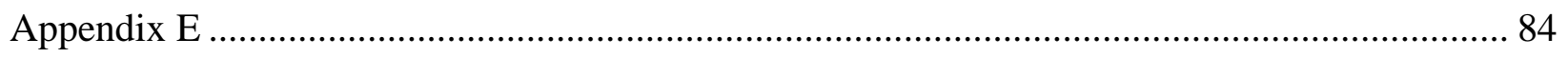

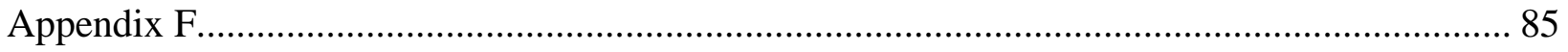

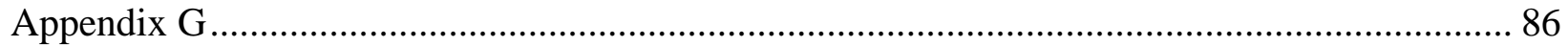

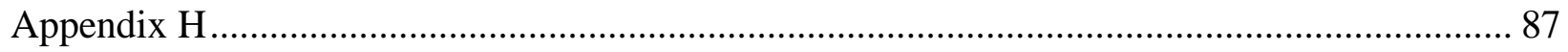

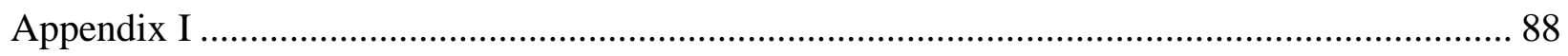

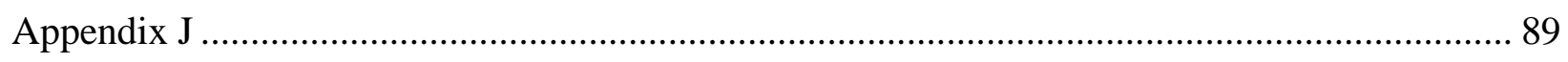

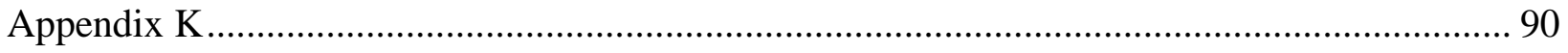

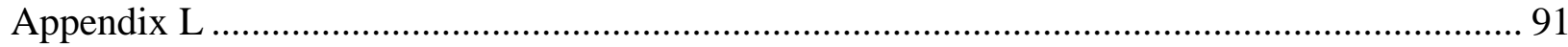

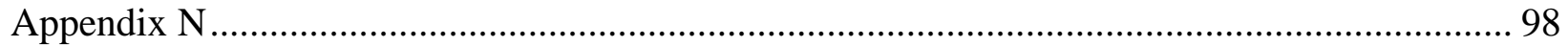

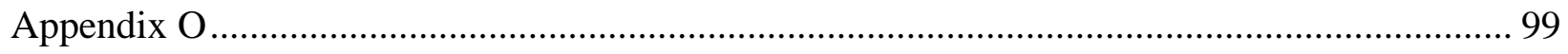

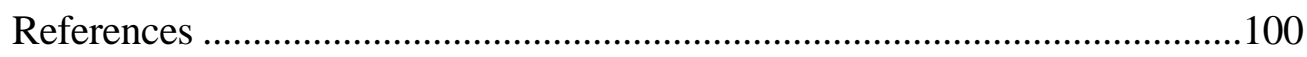




\section{CHAPTER 1}

Overview of chronic diseases and lifestyle programs and the original data source, diabetes prevention and management program 


\subsection{Chronic Diseases}

The emerging pandemic of chronic diseases, also known as noncommunicable diseases (NCDs), are a result of a multitude of factors related to genetics, physiological, behavioral, and environmental causes (Islam et al., 2014). NCDs are the leading cause of mortality worldwide with 39.5 million or $70 \%$ of all global deaths in 2015 accounted for by NCDs (World Health Organization (WHO), 2018). Among the NCDs, $48 \%$ of NCD deaths were premature (before age of 70) in low- and middle-income countries and $80 \%$ of premature heart disease, stroke and diabetes can be prevented. The global burden of NCDs are projected to reach approximately $75 \%$ of all deaths by 2020 (Murray \& Lopez, 1996). Consequently, it is a serious public health threat to both developing and western countries.

In the United States (U.S.), 50\% of all adults (117 million) in 2012 were diagnosed with one or more NCDs and 25\% had two or more NCDs (Ward, Schiller, \& Goodman, 2014). While cardiovascular disease (CVD) and cancer and related deaths accounted for the highest burden $(46 \%)$ in the U.S., more than one-third of adults have obesity or a body mass index (BMI) of $\geq$ $30 \mathrm{~kg} / \mathrm{m}^{2}$ (Centers for Disease Control and Prevention (CDC), 2015; Ogden, Carroll, Fryar, \& Flegal, 2015). Obesity is an established risk factor for many NCDs and is specifically associated with diabetes, hypertension and CVD. Behavioral risk factors of NCDs not only influence the U.S. state health rankings but also have severe social and economic impact on individuals and communities. For example, WV, an entirely Appalachian state, has the second highest rate of obesity with $35.6 \%$ of adults who are obese (BMI $\geq 30$ ) and $68.8 \%$ who are either overweight or obese (BMI $\geq 25)$ (CDC, 2015). Multimorbidity, or the presence of two or more NCDs is high in WV; $40 \%$ of adults are diagnosed with hypertension and $80.6 \%$ report taking medication to control their chronic condition(s) (CDC, 2011).

\subsubsection{Diabetes}

Similar to the rising prevalence in the NCDs, diabetes mellitus is one of the most common chronic diseases significantly escalating globally, nationally and in WV (CDC, 2017). The global prevalence of diabetes has increased from 4.7\% in 1980 to $8.5 \%$ in 2014 in the adult population, and the burden of diabetes continues to rise in the U.S. (from $2.5 \%$ in 1980 to $9.4 \%$ in 2015) (CDC, 2014; CDC, 2017). Currently, according to the CDC (2017), 30.3 million of the U.S. population have diabetes. Additionally, 84.1 million individuals have pre-diabetes and are at high risk for developing diabetes due to an elevated blood sugar level of $100-125 \mathrm{mg} / \mathrm{dL}$. Combined, approximately half of all U.S. adults have abnormal blood sugar or dysglycemia (diabetes or prediabetes). WV ranks first nationally in the prevalence of diabetes with $15.3 \%$ of the population having diabetes and additionally 39.5\% having prediabetes (American Diabetes Association (ADA), 2017; CDC, 2018).

Diabetes is a metabolic disease or condition that results from dis-balance between the demand and production of the hormone, insulin. Insulin is used to convert sugar, starches, and other foods into energy needed for daily life activities and dysfunctions are characterized by hyperglycemia, insulin resistance and relative insulin deficiency (Mollaoglu \& Beyazit, 2009). However, the multifactorial etiology of diabetes is yet to be determined, with genetics, behavioral, and environmental factors being considered. Type 2 diabetes (T2DM), which accounts for $90-95 \%$ of all diabetes cases, is characterized by inadequate insulin production or 
use by the pancreatic beta cells (The Emerging Risk Factors Collaboration, 2010). The high rates of diagnosed T2DM and pre-diabetes in WV can be attributed to many factors, which include geographical isolation, limited or lack of access to quality care, the aging population, and specifically the Appalachian culture of distrust (West Virginia Department of Health and Human Resources, 2012).

\subsection{The Appalachian Region}

Approximately $8 \%$ of the U.S. population or 25 million individuals reside in Appalachia, a region characterized by overall rurality and lower socio-economic status (SES) compared to the rest of the U.S. (Appalachian Regional Commission, 2017). The Appalachian region includes all of WV and parts of 12 other states extending from southern New York to northern Mississippi (Knox, 2014; Paskett et al., 2011). Socioeconomic conditions (i.e., high levels of poverty, high unemployment rates, low-levels of educational attainment, lack of health insurance, and inadequate access to medical care services) are often cited as the contributor to poor health in the region (Shandera-Ochsner et al., 2014; Towers, 2005; West Virginia Health Statistics Center, 2014, U.S. Census, 2015; Halverson, Ma \& Harner, 2004). Furthermore, stereotypes related to common Appalachian values in this region in regard to the poor health indicators of the population include loyalty to family, independence, religion, pride, and minding one's own business and equality (Helton \& Keller, 2010; Towers, 2005; Cantley-Falk, 2002). Consequently, a lot of attention has been given to the Appalachian region through the media and researchers. For example, many "outsiders" have come in and drawn conclusions about this region which could be stereotypical or mischaracterize the region. However, there has been some research that has asked Appalachians what it means to be Appalachian (Helton \& Keller, 2010; Helton, 1995; Bauer \& Growick, 2003; Coyne, Demian-Popescu \& Friend, 2006).

Health beliefs and well-being in the Appalachian region is also shaped by the practice of folk medicine (Cavender \& Beck, 1995). Individuals may use herbal preparations and in-home practices, talk with family members before scheduling a doctor's visit, and tend to consider a hospital as the last option, sometimes thought of as a place where someone goes to die (Cook \& Baisden 1986; Strain, 2002). Consequently, rates of preventive and wellness check-ups and awareness of diabetes risk factors are low as many individuals might not go to the hospital or visit the doctor unless they experience a major health crisis such as a stroke, heart attack, or trouble breathing (Strain, 2002). Furthermore, an illness is typically perceived when the person has symptoms or feels ill. In addition, many of the symptoms are often perceived to be the result of "getting old" or due to fatalism (the belief that present circumstances, future events and even one's health may be beyond human control) (Welch, 2011; Royse \& Dignan, 2011; Hag Hamed, 2017). Hence, individuals might believe that disease and other chronic conditions are going to happen regardless of any prevention or management behaviors. Social relationships can also shape healthcare decisions. Appalachians may be more likely to follow stereotypical gender norms compared to individuals outside of the region (Lewis \& Billings, 1997; Helton \& Keller, 2010; Towers, 2005; Cantley-Falk, 2002) and decision-making regarding health and well-being may be prioritized (Davidov, et al., 2018). Prior studies show that pride and unfamiliarity are the greatest cause of distrust that many Appalachian people feel toward modern medicine (Cavender \& Beck, 1995; Strain, 2002; Lewis \& Billings,1997). Hence, traditional medicine is chosen as 
the first line of treatment due to their familiarity, and unfamiliar and/or untested methods of treatment are generally avoided. Furthermore, pride often prevents them from seeking help from non-family members, especially those that are members of their community.

\subsection{Diabetes, Risk Factors and Complications}

In addition to the socioeconomic and cultural conditions within Appalachia, high rates of unhealthy behaviors (e.g., poor diet, physical inactivity, and smoking) are major contributing factors to diabetes and its complications in this region (CDC, 2017). When an individual has diabetes, they are at-risk for various comorbidities and complications associated with higher diabetes-related morbidity and mortality (Miech, Kim, McConnell, \& Hamman, 2009). In particular, diabetes is associated with numerous micro- and macro-vascular complications that contribute to an increased risk for blindness, stroke, heart disease, heart attack, lower limb amputation, and kidney failure (The Emerging Risk Factors Collaboration, 2010). The frequency and severity of complications are dependent on an individual's type of diabetes (type 1, T2DM or gestational), duration and self-care of the disease that impact diabetes outcomes (Tabish, 2007; WHO, 2018). T2DM is the most preventable in the U.S. and WV (ADA, 2017). The risk factors in WV that increase the incidence or new cases of T2DM are as follows: BMI, physical inactivity, family history, age, history of gestational diabetes, high blood pressure and abnormal cholesterol/triglyceride levels. T2DM can be prevented and controlled with healthy lifestyle behavior and adherence to oral medications and/or insulin (National Institute of Diabetes and Digestive and Kidney Diseases, 2016; DPP Research Group, 2015). Evidence-based lifestyle modifications have shown success for T2DM prevention and management and improved blood glucose levels.

\subsection{Evidence-Based Lifestyle Programs}

Many studies have investigated how to prevent, control, or manage T2DM. Research shows the success of several large-scale evidence-based lifestyle programs. For example, the Swedish Malmo study (5-years, 222 participants) was one of the first lifestyle intervention programs that focused on incorporating intensive diet and exercise modifications (Eriksson \& Lindgarde, 1991). Results demonstrated a reduction in the prevalence of T2DM by over $50 \%$ of participants. Similarly, the Da Qing study in China and the Finnish Diabetes Prevention Study (DPS) examined the effect of a lifestyle intervention in preventing T2DM (Pan et al. 1997; Lindstrom et al., 2003). The Da Qing study (N=577) was implemented in three groups: exercise, diet, or diet plus exercise versus a control group while the DPS $(\mathrm{N}=522)$ was implemented with intensive diet and exercise versus a control group. Both studies found that a combined diet and exercise program significantly reduced the risk of diabetes (Da Quing-42\% and DPS-58\%). Although these lifestyle interventions were considered successful, the generalizability of the results was limited due to the demographic profiles of enrolled participants: the Swedish Malmo study included only Swedish males; the Da Qing study was limited to Chinese individuals in China and the DPS examined Finnish individuals (Eriksson \& Lindgarde, 1991; Pan et al., 1997; Lindstrom et al., 2003).

The DPP, a benchmark U.S. program implemented in the early 2000s and funded by the National Institute of Health (NIH), included individuals from diverse age, socio-economic, racial/ethnic backgrounds and geographical locations (The DPP Research Group, 1999). This 
benchmark program has shown that people with prediabetes who take part in a structured lifestyle change program can reduce their risk of developing T2DM by losing weight, increasing their level of physical activity and committing to heathier eating (CDC, 2017). The impact of this program can last for years and has been found that even after 10 years, people who completed the program were one third less likely to develop T2DM.

\subsubsection{Diabetes Prevention Program}

The DPP was a large, multi-center clinical trial that randomized adults with pre-diabetes to one of three intervention arms: intensive lifestyle intervention, pharmaceutical or metformin therapy, or placebo control (The DPP Research Group, 1999; Kramer, Miller, \& Siminerio, 2014). The intensive lifestyle intervention was delivered by lifestyle coaches and included individual and group sessions; training in diet, exercise, behavior modification skills, and supervised bi-weekly group exercise sessions.

At the three-year follow-up, the DPP resulted in 58\% reduction in their chances of developing T2DM in the lifestyle arm vs $31 \%$ in metformin (Knowler et al., 2009). Likewise, at the ten-year follow-up, the DPP resulted in a 34\% lower incidence of diabetes for the lifestyle intervention group and $18 \%$ in the metformin group compared to the placebo. The cost of the lifestyle intervention was $\$ 3,540$ per participant over three years (Hernan et al., 2003) and viewed as cost-prohibitive for most health plans (Eddy, Schiessinger, \& Kann, 2005). Hence, cost-effective program delivery methods have been explored by previous studies. For example, researchers at the University of Indiana partnered with the local Young Men's Christian Association (YMCA), charitable activities along with an athletic facility, to translate the DPP program to a group-based, community setting (Ackermann \& Marrero, 2007; Ackermann, Finch, Brizendine, Zhou, \& Marrero, 2008). At six and twelve months, the intervention group had a greater decrease in body weight compared to the YMCA standard control group who only received brief counseling and materials $(6 \% \mathrm{v} .2 \%, \mathrm{p}<0.001)$. The authors concluded that a community-based approach, such as the YMCA, was a potential avenue for broad dissemination of the program at lower cost.

Similarly, a modified DPP has been developed and delivered by certified diabetes educators from established community-based diabetes self-management education programs, called the Group Lifestyle Balance (GLB) program (Kramer et al., 2011). Another program used community health workers (CHWs) with well-controlled diabetes who were trained as lifestyle coaches to deliver the DPP through an established diabetes education program in North Carolina (Katula et al., 2011). Results showed a significant decrease in weight, waist circumference, and BMI at 3 months in the entire group $(n=81)$ as well as in those who completed a subset of classes $(\mathrm{n}=68)$. Program participants had significantly greater weight loss $(7.2 \%$ vs. $1.3 \%, \mathrm{p}<0.001)$ and decrease in fasting glucose levels $(-4.3$ vs. $-0.4 \mathrm{mg} / \mathrm{dL}, \mathrm{p}<0.001)$ as compared to the enhanced usual care control group, which only included individual sessions with a registered nutritionist.

Over time, the DPP has been has also tailored for various types of audiences (Jackson, 2009) and settings including worksites, inner cities, rural churches, geographical settings (rural, urban, semi-urban, and medically underserved areas), hospitals/clinics, and YMCAs (Baker, Simpson, Lloyd, Bauman, \& Singh, 2011; Jackson, 2009; Kramer et al., 2014; Neamah, Sebert 
Kuhlmann, \& Tabak, 2016). The various population subgroups included obese, overweight, T2DM, pre-diabetes, and adults, aged 48-60 years from diverse race/ethnicities including White, African-American, Asian, Hispanic/Latino, and Pacific Islander.

\subsubsection{Faith-based Diabetes Prevention Programs}

Studies have also explored the DPP's feasibility in faith-based settings. For example, the CDC Risk Assessment was administered in a rural African American church showing that 28.3\% of the church's population was at high-risk for diabetes (Davis-Smith et al., 2007). The DPP was successful, as it resulted in a mean weight loss of 8.8 pounds in this rural African American population and was sustained over 6 months ( 6.5 pounds) and 12 months (10.6 pounds) of the program. The results suggested that DPP is feasible in rural church settings. However, a lesson learned from this community program was to involve church leadership in the planning and implementation of the program such as including a church member in the program implementation, having pastoral support, and conducting focus groups with church members to guide the process. Specifically, partnerships with church leadership as an equal partner was suggested when considering administrative time and location within the church (Davis-Smith et al., 2007; Levin, 1984; Resnicow \& Campbell, 2004).

Another study by Faridi et al. (2010), also examined the effects of a physical activity and dietary intervention in African American churches. This study examined the effectiveness of the use of community health advisors' (CHAs) or lay health workers for core program delivery to prevent diabetes. The CHAs and a control group of CHAs from a different location were trained on modified DPP lifestyle strategies during a 10-week session series (2 hours per session). The program assessed changes in knowledge of diabetes and dietary intake (total calories, total protein, total carbs, trans fat, mono- and polyunsaturated fats) after the intervention. Although no significant changes were observed in the program outcome measures, researchers asserted that training and compensating CHAs to implement the DPP could provide significant return on investment (McQuiston \& Flaskerud, 2003; Fairdi et al., 2010). Participatory engagement of church leaders and members in program planning, including efforts to culturally tailor the program and address religious values held by the target population may increase the likelihood of positive results in future studies.

A DPP intervention at the Holy Rosary Healthcare facility in Miles City, Montana evaluated the feasibility of translating the DPP lifestyle intervention in a rural community in Montana (Vadheim et al., 2010). A total of 101 pre-diabetic participants, predominantly women, participated in the program. Results showed that the recommended 150 minutes per week physical activity goal was reached by $65 \%$ of the participants. Further, $52 \%$ of the participants completed the suggested $>7 \%$ weight loss goal. The majority of participants also maintained initial weight loss in the after-core sessions (6-12 months of the DPP program) (Vadheim et al., 2010; DPP Research Group, 2002). These results confirmed the feasibility of implementing a group-based DPP in a rural community while still achieving original DPP program goals. In summary, the modified DPP lifestyle interventions administered in rural and faith-based communities have shown effectiveness and success for improving lifestyle changes that reduce the risk for diabetes and blood sugar levels. Yet, to-date, no research has shown the successful translation of the DPP or other evidence-based lifestyle program to address diabetes prevention 
and management in the medically underserved state of WV that has a high prevalence of diabetes and prediabetes.

\subsection{Psychosocial Factors in Diabetes Prevention and Management}

One of the most fundamental strategies in diabetes prevention and control is the willingness of the participants to modify behavior and adhere to recommended diet, physical activity, monitoring blood sugar and medication adherence (AADE, 2008). A large body of research has identified psychosocial factors that could be targeted by interventions to improve diabetes self-management and treatment outcomes (Gonzalez, Tanenbaum \& Commissariat, 2016). Social support, a complex and multidimensional construct, plays a key role in improving participant self-efficacy for behavior changes and has a buffering effect on diabetes distress (Forsythe et al., 2014; Cohen \& Willis, 1985; Sherbourne \& Stewart, 1991; Baek, Tanenbaum \& Gonzalez, 2014). Two broad categories of social support have been explored in prior studies for health behavior changes: (1) support from friends; and (2) support from family (see Chapter 3 ). Having support from family and friends has helped patients cope with their disease and the improvement in self-care and health outcomes. Yet, information on social support available for adults with diabetes or prediabetes is lacking for West Virginians who are disproportionately burdened by dysglycemia (ADA, 2017). Hence, an understanding of as well as addressing barriers to lack of social support, can be beneficial to the success of evidence-based lifestyle programs for improved health outcomes.

\subsection{Community-Based Participatory Research}

One approach that may improve the effectiveness of diabetes programs is communitybased participatory research (CBPR). CBPR is an approach or orientation to conducting research using robust community/patient engagement (Goh et al., 2008). It works to build partnership, mutual trust and respect between researchers and communities as they work together on program planning and implementation (Goh et al. 2009; Kitzman et al., 2017). Through CBPR, it allows for effective community engagement and feedback in the research processes and implementing programs that are meaningful and beneficial to the community. As the first step of CBPR, researchers identify the community and community partners and builds on existing strengths to evaluate the program relevance and modifications (Horn et al., 2006). CBPR allows for improved sensitivity, reliability, and validity of measurement tools (Minkler, 2005) but is a longterm process that requires a mutual commitment from researchers and community members to increase sustainability (Goh et al.,2008). This mutual long-term commitment can result in beneficial outcomes for both community partners and researchers.

However, most lifestyle program researchers usually limit themselves by only using one or two strategies of CBPR to enhance the design, implementation, or sustainability of an intervention (Cargo \& Mercer, 2008; Jones \& Wells, 2007). For example, researchers in one study worked with church leadership to enhance the initial set-up of the program (Davis-Smith et al., 2007), but failed to utilize additional or appropriate CBPR methods throughout the intervention process (Flicker et al., 2007). Investigators implementing diabetes prevention programs often do not utilize or fail to mention their use of CBPR in the creation of research questions, materials, or other implementation techniques. Community stakeholders should be involved in the entire research and program development and implementation process (Fetterman 
\& Wandersman, 2005). If the community members and stakeholders are engaged in the beginning of the planning process, they are more committed to participating in the future (Cargo $\&$ Mercer, 2008). In addition, participatory approaches allow for social and cultural validity throughout the entire research process.

Community stakeholders, who participate in community advisory boards, allow for more effective interventions to be developed and tested. For example, one study used community advisory boards to help design an intervention related to food and physical activity environments in a rural community (Kegler et al., 2012). The use of multiple CBPR techniques increase the translation of research and allow for a better understanding of community concerns (Goh et al., 2008). Administering surveys, conducting focus groups, and interviewing local community members can increase the adoption and sustainability of interventions in the community. Through CBPR, researchers can begin to understand the social, political, environmental, and economic factors related to nutrition, physical activity, and diabetes prevention and management.

Specifically, in chronic disease management, it is often difficult to develop and conduct randomized controlled trials. In addition, randomized controlled trials are not always appropriate or feasible for social and behavioral or health outcome studies (Dougherty \& Conway, 2008). Hence, there is a need to sustain the engagement of health care providers and communities in a participatory process in order to effectively implement public health programming such as those for chronic diseases. This allows for the development and implementation of practice-based research and program evaluation (Green, 2008). An intervention can be successful when it is cost effective with the least amount of resources and therefore community engagement has shown to increase program effectiveness (Wallerstein \& Duran, 2010).

\subsection{Need for Evidence-based Lifestyle Programs in West Virginia}

Given the high rates of diabetes and prediabetes, adherence to healthy lifestyles for both prevention and management of diabetes is a felt need in WV. In addition, limited information exists on psychosocial factors such as social support and perceived benefits and barriers to community-based lifestyle programs in $\mathrm{WV}$, which is a predominantly rural state. This is a problem, as this knowledge can play a significant role in enhancing effective programming that aims to reduce diabetes prevalence and improve the quality of life among WV communities. Furthermore, these factors have been inadequately studied in prior DPP or diabetes selfmanagement programs nationally and in Appalachia (Baker et al., 2011; Jackson, 2009; Kramer et al., 2014; Neamah et al., 2016).

Weight loss and adopting healthy dietary and physical activity habits are important for individuals with diabetes and prediabetes. However, there have been few diabetes programs which have included lifestyle modifications that target both individuals with diabetes and prediabetes in one program although lifestyle modifications help both groups. Hence, targeted weight loss, glucose reduction and improved lifestyle habits can result in better quality of life for those with prediabetes and diabetes. Prior studies implemented evidence-based programs which have stemmed from clinical trials for diabetes and prediabetes separately, but they have not mixed both groups (Baker et al., 2011; Jackson, 2009; Kramer et al., 2014; Neamah et al., 2016). However, for pragmatic trials and where resources are limited, there is no justification to keep 
participants in separate groups. Specifically, a landmark clinical trial in the U.S., the Look AHEAD (Action for Health in Diabetes) trial, has demonstrated that an intensive lifestyle intervention can reduce cardio-metabolic risk factors for T2DM (Belalcazar et al., 2010). Many of the diabetes management strategies focus on lifestyle modification and overlap with the DPP (e.g., healthy diet, being active, stress management, medication adherence etc.). Therefore, the Diabetes Prevention and Management (DPM) program combined prevention and management curricula (to be described in Chapter 1, Section 1.9) was developed for delivery within a population scale in two successful large-scale community trials in India (Balagopal, Kamalamma, Patel, \& Misra, 2008; Balagopal, Kamalamma, Patel, \& Misra, 2012).

Additionally, few modified DPP programs (and none in WV) have supplemented the use of qualitative data collection with behavioral and clinical measures to provide an in-depth understanding of the program delivery and subsequent outcomes, adequate dissemination, and specifically, the understanding of participant social support and participants' perceptions of program benefits and barriers in diabetes prevention and management programs.

Furthermore, qualitative data collection techniques, in combination with CBPR, have led to a better understanding of lifestyle programs such as uptake of program and participant experiences (Cargo \& Mercer, 2008; Minkler, 2005; Horn et al., 2006). Furthermore, qualitative and CBPR methods allow researchers to tailor curricula for diverse communities to enhance the probability of programmatic success. Modifications may include changes based on relevant cultural characteristics of the target population, appropriate dietary and physical activity modifications, and links to community resources. Currently only $50 \%$ of individuals in WV with diabetes have received diabetes self-management education (DSME) (Misra \& Sambamoorthi, 2017). This is problematic as this could contribute to lack of glycemic control and unhealthy behaviors leading to high morbidity and mortality from diabetes. Specific and relevant information that is tailored to the needs of this population is needed to improve research and practice translation and increase dissemination of diabetes prevention and management education in the state.

Moreover, there is a dearth of information in this field about benefits and barriers associated with participating in lifestyle programs. Hence, an understanding of benefits and barriers of participating in DSME as well as lifestyle programs may explain the limited number of DPP and other diabetes self-management programs in WV (CDC, 2018; Evidence-Based Leadership Council, 2018; Self-Management Resource Center, 2018; National Council on Aging, 2018). In addition, few diabetes research studies in WV have used a rigorous approach such as the use of multiple methods (i.e., quantitative and qualitative) to provide a comprehensive and a deeper understanding of the context in which psychosocial factors may influence diabetes risk and its management for program effectiveness.

\subsection{Specific Aims and Research Approach}

The long-term goal of this research is to enhance current understanding of factors that singularly and additively contribute to prevention and/or management of diabetes. To achieve this goal, the overall objective of this research was to examine the relationship between perceived social support, benefits and barriers of program participation and clinical outcomes 
(A1c, LDL, HDL, and weight loss) of a 2-year pilot DPM program implemented in two churches in Morgantown and Charleston, WV. The DPM program used a modified DPP program (12months, 22-session) with diabetes self-management added to the curriculum (AADE, 2008). The central hypothesis was that perceived social support and program benefits, and lower barriers to behavioral modifications will increase healthy behavior changes among DPM participants. The rationale underlying the proposed research was that rigorous translational research that incorporate psychosocial factors with a multiple methods approach can provide a better understanding of lifestyle program effectiveness in WV. The results were expected to help: (1) inform critical prevention and management strategies to address diabetes disparities in WV communities; and (2) allow further tailoring of the DPM and other diabetes programs for effective behavior modification and sustainability. The DPM program sample comprised of 94 adults with diabetes and prediabetes. This research focuses on the following three specific aims:

Specific Aim 1: To examine whether participant's program attendance mediate the relationship between participants' perceived benefits and barriers to program participation and percent body weight. Hypothesis: Program attendance significantly mediates the relationship between benefits and barriers and percent body weight.

Specific Aim 2: To assess the contribution of social support on behavioral modification and improved clinical outcomes among participants of the DPM program. Hypothesis: Participants with higher perceived social support from family and friends for behavioral changes (diet and physical activity) will have significantly higher program attendance and improvements in diabetes outcomes i.e., cholesterol (lower LDL and higher HDL) and A1c.

Specific Aim 3: This exploratory aim was related to Specific Aims $1 \& 2$ to qualitatively explore DPM participants' social support experiences for behavioral modifications and program benefits/barriers, using focus groups. Since this aim was qualitative and qualitative aims do not have hypotheses, no hypothesis was proposed.

The proposed project aimed to fulfill a need in rural Appalachia and was built on prior successful community-based DPM programs in rural India using CHWs. However, this study used trained Health Coaches (HC) model of delivery and used quantitative and qualitative techniques to construct predictive models unique to WV adults with diabetes and prediabetes (Lanese, Dey, Srivastava, \& Fingler, 2011). In addition, this research builds on my prior GLB training in 2012 at the WV Bureau of Public Health (WVBPH) as well as experience as the Program Coordinator and trained Health Coach for the pilot DPM program (from July 2014 to October 2016) where I worked closely with the Principal Investigator (Dr. Ranjita Misra) and twelve other Health Coaches for implementing the DPM program in Morgantown and Charleston, WV.

Network connections such as working with the WVBPH and other diabetes community program leaders in my current role as the Project Coordinator, OHSR in the WVU SPH and my expertise with diabetes programs in $\mathrm{WV}$ uniquely positions me to complete the specific aims for this study. This study fills a critical and immediate need for new and creative approaches to 
translate and tailor evidence-based programs in WV. In addition, use of multiple methods approach represents a novel way of understanding and evaluating program feasibility and effectiveness of the pilot DPM program.

\subsection{Curriculum}

The DPM program is a 22-session program that included 16 core sessions and 6 post-core sessions, implemented over 12 months. The DPM was founded on the GLB with additional AADE7 added to the curriculum (Ingels, Misra, Stewart, Lucke-Wold, \& Shawley-Brzoska, 2017; Lucke-Wold, Shawley, Ingels, Stewart \& Misra, 2016). The original DPP (16-session, 12 months) curriculum, developed by the National DPP (NDPP), which is part of the CDC (DPP Research Group, 2002; CDC, 2018), is based on the Social Cognitive Theory and has established standards to assure quality. The program emphasizes goal-setting and self-monitoring to make lasting improvements in nutrition and physical activity; moderate weight loss; building selfefficacy and social support for sustainable lifestyle changes; and overcoming barriers to maintaining weight loss and lifestyle changes. The lifestyle balance goals are moderate weight loss of $7 \%$ of baseline body weight and completion of 2.5 hours of brisk, physical activity each week (Kramer et al., 2011). Research has shown that participants of DPP, modified DPP and GLB, were able to successfully prevent the early onset of diabetes, reduce diabetes-related complications, and improved health outcomes by setting attainable goals and engaging in healthy behaviors.

Similar to the DPP, the GLB is a comprehensive lifestyle behavior change program with a highly prescribed curriculum on intense lifestyle modification that is administrated in a group setting. Initially, the curriculum was offered in 12 GLB core sessions over a period of 12-14 weeks. Following the core sessions, the transition sessions were offered at a lower frequency; presented bi-weekly or monthly depending on site scheduling. In addition to the core and transition sessions, six monthly sessions were offered to provide ongoing support and accountability throughout the remainder of the year.

The DPM program curriculum was developed by a core team of experts (Physician, Certified Diabetes Educator, Clinical Nutritionist, Public Health, Epidemiology, and Health Behavior) and the multidisciplinary team combined and modified two evidence-based curricula to finalize a 22-session, 12-month DPM curriculum (see Appendix A). Three of the 22 sessions focused on diabetes management (AADE7 framework) and 19 sessions focused on lifestyle behaviors and modifications (AADE, 2008). The DPM intervention consisted of face-to-face (group) educational sessions taught by the trained HCs and experts (60 minutes per session; weekly for first 12 sessions, bi-weekly for next 4 sessions and monthly for the last 6 sessions). Each session included a specific lifestyle behavior, skill building activities, group sharing and problem-solving regarding behavior change, $\mathrm{Q} \& \mathrm{~A}$ and encouragement to develop a specific behavior-change goal and action plans for the following week. Additional HC weekly educational booster questions were provided to initiate (and standardize) weekly conversations during follow-up communications with each participant via telephone/text/email (as per the participant preference). Through these contacts, the trained HCs provided one-on-one motivation, skills for problem solving, and linkages to needed education and health care 
resources. All sessions were video recorded and those participants who missed the session could review it using a closed YouTube link created for the participants.

\subsection{Health Coaches}

Based on prior successful program delivery using $\mathrm{CHWs}$, the current program used trained HCs to deliver the core curriculum. A trained \& multidisciplinary group of HCs included graduate and undergraduate students. Thirteen (HCs) were selected (six in year $1 \&$ ten in year 2; three HCs overlapped between the two years) by a formal process that included an application and interview. The HCs were from WVU and Marshall University, from various majors: nursing, nutrition, physical activity and sport sciences, health communication, medicine, and public health, and varied educational levels (BS, MS, $\mathrm{PhD}$, and $\mathrm{MD}-\mathrm{PhD}$ ), selected through a competitive application process to examine their fit based on interest, background, and levels of experience in lifestyle interventions and community-based research.

The DPM study was piloted in one site (Morgantown) in year 1 and expanded to 2 sites in year 2 to allow for flexibility and tailoring of the educational sessions and data collection schedules. Before providing lifestyle intervention in the pilot trial, the HCs received an intensive 3-day training and 1-day refresher (mid-program) by an interdisciplinary team of experts to enhance their knowledge and skills on lifestyle interventions, epidemiology of diabetes, Appalachian health disparities, and key elements of self-management approaches and lifestyle curriculum. The training also helped familiarize the HCs with the measures and standardization of data collection activities (administering blood pressure readings, weighing and measuring height, along with survey administration), and data entry. We used prior pedagogical techniques including didactic presentations, role-playing, discussion, and behavioral skills and mock presentations. In addition, 26 biweekly HC meetings (2 hours each) provided additional opportunities for information, mock session presentations and programmatic discussions. The trained HCs helped recruit participants, deliver the lifestyle intervention, communicate with participants each week, help with data collection/process and outcome evaluation; a subgroup was part of the DPM Research Committee which reviewed and analyzed data based on their interest areas.

\subsection{Study Design and Data Collection Sites}

The DPM program utilized a prospective pre-post intervention study design to evaluate the delivery of an evidence-based, non-pharmacological, behavioral, lifestyle intervention for individuals with pre-diabetes and diabetes. Data was collected prospectively at the Woodland Methodist church in Morgantown, WV for year 1 and 2 as well as at the Village Chapel Presbyterian church in Charleston, WV for year 2 only. These two churches were centrally located to ensure access to the target population from the two cities and its surrounding areas and consisted of a gym, kitchen, several rooms and an open hall for delivery of the educational program.

\subsection{Eligibility Criteria}

Individuals who were at risk for developing diabetes (i.e., in the prediabetes stage) or were diagnosed with diabetes, based on self-report, survey screening questions or fasting blood glucose, were recruited for the study. Eligible individuals were aged 18 years or older with a 
BMI of equal or greater than $24 \mathrm{~kg} / \mathrm{m} 2$, were able to complete the Surgeon General's recommended physical activity of at least 150 minutes per week and complete the intervention and all assessments. All participants were encouraged to contact their primary care provider (if they had one) regarding their participation in the study. There were no exclusions by gender, race/ethnicity, co-morbidities or medication intake. Exclusion criteria included those who were less than 18 years old, currently pregnant (due to weight loss restriction), inability to provide informed consent due to mental illness or cognitive impairment or have any condition that requires physical activity limitations. These criteria are consistent with those often used in the DPP or its modified interventions. The project staff (HCs and Program Coordinator) assessed participant eligibility using a diabetes risk factor survey.

\subsection{Participant Screening and Recruitment}

All recruitment procedures complied with WVU Institutional Review Board and the Health Insurance Portability and Accountability Act (HIPAA) guidelines. Previous successful recruitment strategies were used for participant recruitment. In addition, the project team developed a church advisory board (CAB) (in year 1) and used their guidance for planning and tailoring the DPM intervention program to meet community needs. The CAB helped ensure that all aspects from the planning, recruitment, advertising, educational materials, and the lifestyle intervention were participant-centered and culturally relevant.

Potentially eligible participants were recruited from the greater Morgantown and Charleston, WV areas using strategies that included: convenience and snowball sampling, advertising in internet and print media, health fairs at the local mall, posting flyers and brochures at Federally Qualified Health Center (FQHC) and free clinic waiting rooms, and in public places such as community bulletin boards (physical and electronic), physician's offices, churches, and clinic waiting areas. These two areas have several hospitals, FQHC, free clinics, churches and other community-based organization (e.g., YMCA) from where participants were successfully recruited for the program. Study staff also gave presentations regarding the program to interested lay and professional groups in the area, including clinical, church groups, and regional wellness organizations (e.g., WV Wellness Council).

Recruitment materials described the opportunity to participate in a program designed to help individuals with pre-diabetes and diabetes begin a lifestyle program. The advertisements also described the experiential nature of the program as well as the opportunity to earn $\$ 100$ in gift cards ( $\$ 25$ provided every 3 months based on program participation) for a local grocery store to compensate for their time and travel for participation in the program.

\subsection{Study Setting}

As previously detailed the DPM was implemented at two community settings (churches); in Morgantown, WV and Charleston, WV. Both churches are located centrally and offer large fellowship hall, private rooms, kitchen and even a gym (in Morgantown) that is ideal for program. The study team has a strong collaborative relationship with both these churches and had worked with their advisory boards to discuss and finalize the pilot DPM program recruitment, program materials, and clinical (fasting blood work) sessions. The two sites were 
similar in socio-demographic characteristics and 17\% families live at or below the 2016 Federal Poverty Level $(\$ 24,300 /$ year for a family of four).

\subsection{Study Procedures}

\subsubsection{Participant Consent}

A HC and/or the Program Coordinator explained the program and answered any questions that the participant may have had regarding the research and their participation. Participants were given a copy of the consent form to take home. The consent form was signed at the church and was witnessed by a HC.

\subsubsection{Study Measures and Data Collection}

Data on behavioral, anthropometric and clinical outcomes was collected at baseline, mid- and end of the program (details in 2.7.3).

(a) Cardiometabolic Measures: Participants were instructed to fast for 8-10 hours before data collection for fasting serum blood, collected by trained Phlebotomists. Outcome measures included glucose (or HbA1c), lipid profile (total, HDL and LDL cholesterol, and triglycerides), as well as blood pressure. Clinical laboratory testing was provided by the Mon General Hospital consistent with established standards.

(b) Lifestyle Measures: This included dietary intake and physical activity. Participants' were required to have a commitment for making changes in diet and physical activity, regularly monitoring food intake and exercise and attending program sessions. Participants were weighed at each session and attendance was recorded. Participants were asked to selfmonitor diet (using food logs and the CalorieKing book), physical activity (using pedometers), and weight throughout the program. Self-monitoring is an important part of the DPP program as it helps monitor progress and promotes self-efficacy for lifestyle changes. In addition, weekly food logs were reviewed by HCs and appropriate, culturally tailored feedback were provided to improve their diet and physical activity. HCs followed up with participants weekly on their diet and physical activity via participants' preferred communication method: call, text or email. HCs communications with their participants were tracked using a database for program fidelity.

\subsubsection{Baseline, Mid-Program and Post-Program Assessments}

Data on core outcomes (behavioral, anthropometric, and clinical factors) were collected at baseline, 6-months and 12 months. Trained project staff (HCs, volunteers and other project team members such as the Program Coordinator and Principal Investigator) completed all study assessments. In addition to fasting bloodwork, all anthropometric assessments (height, weight, waist circumference, percent body fat) were conducted in-person in private rooms at the two community sites. Weight metrics and blood pressure were taken twice to ensure consistency and accuracy of readings. Baseline program assessments were completed immediately prior to the intervention start date, and the last two assessments were completed at six and twelve months of the program. Anthropometric and clinical measures were supplemented with surveys during pre-, mid-, post-assessments (i.e., at 0, 6 and 12 months) in order to assess behavioral impacts and outcomes. Surveys included perceived social support for diet and physical activity, health behavior (diet and physical activity), and socio-demographic characteristics. Each assessment 
visit required approximately one hour. Upon completion of the 6- and 12-month intervention, participants also completed a program evaluation questionnaire, designed to assess satisfaction with the DPM program, and perceived barriers to and facilitators of positive behavioral change. Program adherence was determined by measuring class attendance, completion of follow-up phone calls, program assessment, self-reported food and physical activity logs and targeted behavior goals.

\subsection{Sample Size}

Ninety-four adults with diabetes and prediabetes completed the pre-screening survey and agreed to participate in the DPM program. While all the ninety-four participants started the DPM program, several dropped out in the first 1-3 weeks due to extensive time commitment for the 12-month program and/or had family/job/travel challenges to attend the sessions for the 12month period. Eighty-three participants completed the DPM program's baseline assessments (clinical, anthropometric and surveys) but only 68 completed all the baseline, mid-program and final program assessments. However, for this project's quantitative aims (aim 1 and 2), there was missingness for some of the clinical and survey data, which was taken into account with each aim's analyses.

\subsection{Participant Barriers and Satisfaction using Focus Groups \\ 1.17.1 Focus Group Development}

Using the RE-AIM framework, we assessed participants' experience and satisfaction of the DPM program. This framework was developed by Glassgow and colleagues as an evaluation/planning tool to guide program planning through considering the strengths and weaknesses of lifestyle interventions (1999; 2001). RE-AIM stands for Reach, Efficacy, Adoption, Implementation and Maintenance. The framework was used to develop focus group scripts to evaluate the DPM program's potential for translation to WV communities; the effectiveness of the educational sessions/intervention; engagement in program sessions and follow-up calls; likelihood of lifestyle behavior changes and sustainability over time; preferred mode of communications by participants; and barriers and deterrents for successful behavioral modifications (Glassgow et al., 1999). These questions were developed to help capture in-depth information on the program and contextual factors that facilitate or hinder successful implementation.

All guides included a set of identical core questions as well as questions specific to the focus group audience. A total of three focus group guides were developed, utilizing the RE-AIM framework, for each of the following audiences: $\mathrm{CAB}$, participants and health coaches. The moderator utilized the guides and applied qualitative research expertise to probe for additional information as deemed necessary. The goal of the focus group guide was to gain insight and information to perceived benefits and barriers of the program

A cover letter was distributed to all focus group participants prior to the focus groups. The letter provided an overview of the focus group format and process. Focus group participants were notified that answering questions was not required and that responses were kept confidential. It was also disclosed that the discussion would be audio-recorded and, therefore, 
anonymity could not be guaranteed. The planning process and implementation took place once we received WVU IRB approval with an exempt protocol.

\subsubsection{Recruitment and Logistics of Focus Groups}

Focus groups were scheduled largely based on convenience to interested participants. Approximately, 5-10 individuals were recruited per focus group with a total of six focus groups completed in 2 years. The focus groups were conducted with the following groups of participants:)1) Year 1 Morgantown Participants, CAB and HCs, 2) Year 2 Morgantown Participants and HCs, and 3) Year 2 Charleston Participants and HCs. The focus groups took place for 60-90 minutes in private rooms in the following locations: Woodland United Methodist Church (Morgantown DPM program site), Village Chapel Church (Charleston DPM program site) and on WVU Health Sciences Center campus (for HC focus groups).

The focus groups were held immediately following a regularly scheduled DPM program session, after their church service and after a HC meeting. Preliminary participant recruitment was conducted during the DPM program sessions for the focus group. For the CAB focus group, recruitment was initiated by the pastor of the church, who submitted potential names to the $\mathrm{HCs}$ and Program Coordinator. In addition, all HCs were requested to participate in the focus group. Since all HCs were WVU and Marshall students, the focus group was planned in a private room on campus.

Interested participants were asked to complete a sign-up sheet. Follow-up phone calls were made to verify participation prior to the focus group and potential participants were given a description of the focus group process, overall purpose, expected outcomes and were also notified of the $\$ 20$ gift card incentive.

The focus groups were moderated by Dr. Delores James from the University of Florida and Dr. Danielle Davidov from WVU, experts in qualitative research and focus group interviews. Dr. James and Dr. Davidov were skilled in group discussion and used pre-determined questions that were reviewed by the investigators. Dr. Peter Giacobbi and a few WVU SPH student workers assisted in handling the logistics, taking notes, and monitoring the audio recording equipment. 
CHAPTER 2

Identifying the benefits and barriers of a diabetes prevention and management program in a West Virginian community 


\subsection{Significance}

Lifestyle and diabetes education programs have shown sustainable improvements in health behaviors and outcomes and are often recommended for achieving glycemic control (Osborne et al., 2010). These programs effectively assist individuals with diabetes and prediabetes to prevent, manage and reduce complications in cost-effective ways. Yet, new and creative community-based and culturally appropriate diabetes programs are lacking in WV communities. In addition, to develop and implement culturally tailored and evidence-based programs, a better understanding of the participants' perceived benefits and experiences as well as barriers to program engagement and behavior modifications is also necessary for sustainability. A benefit (or facilitator) is defined as a positive item, activity or action that results in promoting a healthy behavior and a barrier is considered a negative action that could hinder the engagement in healthy activities or behaviors (Hansen, Landstad, Hellzen \& Svebakl, 2011).

For example, a follow-up study to the DPP of 1,074 participants (national sample) who received the lifestyle intervention identified barriers to weight loss and physical activity (Venditti et al., 2014). At the beginning of the program, each participant was matched with a $\mathrm{HC}$ who provided support, guidance, and tools during individual meetings throughout the program. After each client meeting, the HCs coded barriers to weight loss or activity. The five most common barriers to weight loss were: problems with self-monitoring, social cues (friends or family pressure), going on vacation or holidays, too little activity, and internal thoughts or moods (selfdefeating thoughts). They also found that barriers were reported in a higher proportion of participants in the second half of the program, showing that barriers tend to increase with the length of the program. For physical activity, the five most common barriers were: internal thoughts or moods, social cues, time management, injury or illness, and access or weather preventing activity. While there was some overlap between barriers to weight loss and increased physical activity, these results showed that these are two different behaviors with unique barriers. The strength of this study was a large, diverse sample that was followed for 12 months, yet all individuals who were pre-diabetic and chose to participate. Hence, there could be other barriers that could have kept individuals from participating.

A second study of 245 low-income women in Minnesota who participated in a supplemental nutrition program for women, infants, and children (WIC) were surveyed about their interest in and barriers to joining the weight loss program (French, Jeffery, Story \& Neumark-Sztainer, 1998). Overall, cost and availability of childcare were the most frequently cited barriers among participants and non-participants, showing that there exists some similarity in barriers in both groups. However, lack of time, family duties, and conflict with work schedule were significantly more likely to be a barrier for those not interested in participating in the program. This suggests there are significant differences between participants and nonparticipants.

Implementation of the various modifications of DPPs in real-world settings have identified some program benefits and barriers. Previous studies suggest that attrition can be related to the individuals' perceptions of their likelihood of developing diabetes based on personal/family history, incentives provided, and their readiness to change for their well-being (Ali, Echouffo-Tcheugui, \& Williamson, 2012). Program attrition was also related to the number 
and frequency of the sessions attended, which can be associated with the magnitude of weight loss. For example, more core sessions offered with greater number of participants attending could equal to a greater amount of weight loss, which could in turn result in a higher attrition rate (Ali, Echouffo-Tcheugui, \& Williamson, 2012; Jeffery, Thompson, \& Wing, 1978; Kramer, Jeffery, Snell, \& Foster, 1986).

While prior studies have focused on how to prevent T2DM, reduce weight, and increase physical activity, programs that target individuals with diabetes and those in the Appalachian region may result in other unidentified barriers. A systematic review of focus groups with 30 individuals with T2DM revealed that diet compliance, pain during physical activity, social pressure, and symptoms of hypoglycemia were key barriers to self-care of diabetes (CaroBautista et al., 2014). These results also reveal some overlap between DPP programs, weight loss, and prevention, but more research is needed to validate barriers and benefits of program participation that may exist across diverse population groups. Elements of DPP that contribute to the success of the program (e.g., diet, physical activity, stress management) can benefit individuals with diabetes and prediabetes.

Some diabetes education and lifestyle programs are currently available in WV. However, rigorous research directed to influence patient behavior and outcomes (such as weight loss) is currently lacking in West Virginia, a state most burdened by diabetes. In addition, there are limited resources and health professionals for providing diabetes education to patients in this medically underserved state. Hence, alternative models of providing diabetes education and lifestyle modifications such as healthy dietary habits, physical activity and coping with stress can benefit both individuals with prediabetes and diabetes. Non-pharmacological lifestyle interventions, such as the modified DPP and others emphasize on modifiable factors that benefit individuals with diabetes and prediabetes to prevent or manage this chronic condition and mitigate the barriers. Therefore, the aim of this study was to explore the benefits and barriers to the DPM intervention targeting individuals with prediabetes and diabetes in WV. More specifically, the mediating effect of participant's program attendance was assessed for perceived benefits and barriers and percent body weight with the following hypothesis: higher programmatic benefits and lower barriers is associated with program attendance and percent body weight.

\subsubsection{Innovation}

This study was one of the first to:

- To assess the perceived benefits and barriers of an evidence-based DPM program in WV, specifically elements of DPP that contribute most to the successes and barriers to diet, physical activity, and stress management

- Assess the relationship between program benefits and barriers, program attendance and percent body weight.

\subsection{Methods}

A secondary data analysis was conducted to identify the participants' perceived benefits and barriers while also assessing the relationship between program benefits and barriers with 
attendance and percent body weight in the DPM program. Data for the DPM program (larger study) was collected to evaluate the feasibility and effectiveness of the program at the baseline, mid-program (at 6 months) and post-program (at 12 months). As mentioned previously in Chapter 1, anthropometrics, bloodwork and survey data were collected during the assessments.

Benefits and barriers were assessed using a survey questionnaire developed by the researcher to examine participant perceptions of the DPM program. The purpose of the survey was to: 1) determine their barriers for engaging in the program, 2) determine the benefits reported for program participation, and 3) assess the satisfaction with various components of the program (if any). This project included an assessment of benefits and barriers by participants at mid-program (6-months) and end of program (12-months). Individuals had the opportunity to rate specific benefits, barriers, provide suggested improvements, and identify the most interesting components that may lead to improvement of community-based diabetes lifestyle programs, using open-ended questions.

This research study examined how program attendance mediated the relationship between benefits and barriers and percent body weight while controlling for demographic characteristics. The effect of benefits and barriers on percent body weight varies depending on program attendance. The null hypothesis was defined by no relationship with percent body weight and benefits and barriers based on program attendance. The alternative hypothesis purports that there was a relationship between percent body weight and benefits and barriers based on program attendance. To test this hypothesis, multiple linear regression statistical tests were used.

\subsubsection{Measures}

\subsubsection{Demographics}

Selected variables were chosen from the larger DPM program dataset. A DPM program participant ID was created for anonymity, with four components: first letter of first name, first letter of middle name, birth month, and birth day. This participant ID allowed anonymity but provided the investigators the ability to connect the survey with demographic information collected at baseline. Demographic questions included the following information of the participant: age, household size, gender, education, marital status, diabetes status and income.

The participant's date of birth was used to calculate the age of each participant. This variable was used as a continuous variable. The number of members in their household was provided and was used as a count, ordinal variable. The categorical variable, gender, was selfidentified as either female and male. For coding purposes, females were categorized as a 0 while males will be categorized as a 1 . The categorical variable, marital status, was coded: 1) currently married and 0) not currently married. The categorical variable, education level, was coded: 0) high school graduate/GED and 1) college degree or higher. Education level has been used in prior DPM programs and hence used for this pilot study. The categorical variable, diabetes status, was identified into having prediabetes or diabetes based on participants' self-reported medical history and supplemented with A1c values (diabetes $\geq 6.5$; prediabetes 5.7 to 6.4). For coding purposes, those with diabetes were categorized as a 1 while those with prediabetes were categorized as a 0 . The categorical variable, income, was coded: 0 ) less than or equal to $\$ 50,000$ 1) greater than or equal to $\$ 50,000$.

\subsubsection{Predictor and Outcome Variables}

Program attendance was computed by summing attendance for all 22 sessions and percent attendance was calculated by dividing the total sessions attended by participants by the 
total number of program sessions and multiplying it by 100; a minimum of four sessions was used for this calculation, which is based on the DPP Recognition Standards. Weight was measured at baseline, mid-program and end of program in addition to the 22 program sessions. Hence, participants who attended the educational sessions had their weights tracked during the program at each week for the $1^{\text {st }} 3$ months, bi-weekly for the $4^{\text {th }}$ and $5^{\text {th }}$ month and monthly for the $6^{\text {th }}$ to $12^{\text {th }}$ month. The first and last weights recorded for each participant during months 1 and 12 were used to calculate this measure. Percent body weight was calculated for all participants from baseline to post-program by taking the baseline weight minus the post-program weight dividing that by baseline weight and multiplying by 100 .

\subsubsection{Benefits and Barriers Survey}

The benefits and barriers survey was developed and implemented by the researchers involved with this project (Shawley-Brzoska \& Misra, 2018) (see Appendix B and C). For this secondary data analysis, 18 survey questions were used, these were modified from two reliable and valid surveys i.e. the Community Health Awareness of Diabetes (CHAD) and the Summary of Diabetes Self-Care Activities (SDSCA) measure (Toobert, Hampson \& Glassgow, 2000; Agarwal et al., 2013). The survey questions identified the benefits and barriers of the DPM program. The benefits and barriers questions used Likert response items ( $1=$ strongly disagree, $2=$ disagree, $3=$ neutral, $4=$ =agree, and 5=strongly agree). These items were summed to compute an average score of benefits and barriers, separately. The barriers questions were reverse coded: (5=strongly disagree, $4=$ disagree, $3=$ neutral, 2) agree, and 1) strongly agree). Therefore, higher scores for benefits indicate more benefits and higher scores for barriers indicate lower barriers to explain their overall satisfaction in the program. All "not applicable" and "blank" responses were recoded to missing data. The first observation (completed at 6 months and/or 12 months) from the participant was used for analysis.

\subsubsection{Statistical Analyses}

To pilot test this newly developed tool, Cronbach's alpha was used to assess reliability or internal consistency of the scale (Shawley-Brzoska \& Misra, 2018). Percent body weight was the dependent (continuous) variable. Age and household size was analyzed as the independent, continuous variables. Education level, diabetes status, income, and gender were analyzed as the independent, categorical variables. Benefits and barriers were analyzed as the primary predictor and independent, continuous variable. Program attendance was analyzed as the mediator, continuous variable.

Data was analyzed using Statistical Package for Social Sciences, Version 25 (SPSS) (IBM Corp., 2017). However, prior to importing data into SPSS, the data was entered in Microsoft Excel and cleaned. Specifically, all the program data was double entered and compared for missingness. All analyses accounted for the drop rate, calculated from participants who completed a baseline and post-program assessment. Participants who had not completed a baseline and/or post-program assessment were excluded from all analyses. Group differences were assessed for the Morgantown and Charleston sites and by diabetes status (prediabetes and diabetes). The multiple linear regression equations listed below in Table 2.1 were used for this study: 
Table 2.1

\begin{tabular}{|l|l|}
\hline Models & Regression Equations \\
\hline 1 & $\mathrm{Y}=\mathrm{B}_{0}+\mathrm{B}_{1} \mathrm{X}+\mathrm{e}$ \\
\hline 2 & $\mathrm{M}=\mathrm{B}_{0}+\mathrm{B}_{1} \mathrm{X}+\mathrm{e}$ \\
\hline 3 & $\mathrm{Y}=\mathrm{B}_{0}+\mathrm{B}_{1} \mathrm{M}+\mathrm{e}$ \\
\hline 4 & $\mathrm{Y}=\mathrm{B}_{0}+\mathrm{B}_{1} \mathrm{X}+\mathrm{B}_{2} \mathrm{M}+\mathrm{e}$ \\
\hline
\end{tabular}

$\mathrm{Y}=$ Percent body weight, dependent variable, what is being predicted or explained, $\mathrm{M}=$ Program attendance and $\mathrm{X}=\mathrm{Benefits}$ or Barriers, independent variables that is explaining the variance in $\mathrm{Y}$ and $\mathrm{e}=$ error term

While 89 participants (unduplicated) were enrolled in the DPM program, the current sample size across regression analyses included 70 participants with complete data on the main variables of interest. Descriptive characteristics for the total sample are provided in Table 2.2. To examine the mediating effect of program attendance on benefits and barriers and percent body weight after controlling for the covariates, multiple linear regression using the Baron and Kenny method was conducted (Baron \& Kenny, 1986). The Baron and Kenny method was chosen due to it being the most widely used technique to assess mediation. The control variables were held constant in the model testing. Each independent variable was individually added to the model to be examined for contribution and significance in predicting percent body weight.

The significance level was set to $p<0.05$. Sample size appropriateness was determined for each independent variable. Correct model specification was conducted by examining the bivariate relationship between the dependent variable (percent body weight) and each independent variable (attendance, benefits, barriers, age, educational, diabetes status, salary, household size and gender). Based on theoretical knowledge, all relevant variables were included. If any of the independent and control variables were not significant ( $p>0.05)$, they were excluded from further analyses.

For benefits and barriers separately, four standard regression models based on the Baron and Kenny techniques were analyzed to determine the variance accounted for by program attendance. Model 1 included percent body weight (outcome variable) and the independent variable (benefits or barriers). Model 1 involved percent attendance (outcome variable) and the independent variable (benefits or barriers). Model 3 included percent body weight (outcome variable) and independent variable, program attendance. Model 4 involved the relationship between benefits or barriers and program attendance on percent body weight (outcome variable). Standardized coefficients/beta adjusted R-square were reviewed, and p-values were assessed for statistical significance. To examine variance in the outcome variable (percent body weight) by using the individual predictors, the adjusted R-squared was analyzed. The R-squared value was used to determine whether program attendance and benefits and barriers were good predictors of variance in percent body weight. In addition, sensitivity analyses were conducted to determine how the independent variables (benefits, barriers and program attendance) impact the outcome variable (percent body weight) (Appendix F).

\subsection{Results}

Table 2.2 presents descriptive statistics on the total sample $(\mathrm{n}=89)$. Participants were primarily female $(73 \%)$ and ranged in age from 20 to 83 years (mean $=58.51$ years, $\mathrm{SD}=0.26$ ). 
Participants reported higher socioeconomic status levels; the majority of participants had a college degree or higher $(69.7 \%)$ and a mean individual income of $<\$ 50,000(45 \%)$. The majority of participants $(63 \%)$ were currently married and lived with an average household size of $1.24(\mathrm{SD}=0.30)$. Two-third of the participants or $62 \%$ had diabetes and $38 \%$ had prediabetes. No differences were found between program sites or diabetes status confirming the assessment of the sample as homogeneous.

Descriptive information (percentages) for the predictor and outcome variable i.e., for attendance and percent body weight are also presented in Table 3.2. Mean attendance was 60.8 percent $(\mathrm{SD}=27.6)$, which was rewarding for a lifestyle program in WV. Mean percent body weight (or weight change percentage) was $1.74 \%(\mathrm{SD}=5.08)$. Participants had a baseline average weight of 217.6 pounds and post-program average weight of 208.2 pounds. This suggests that individuals were hovering around 10 pounds of weight loss or sustained weight during the program period.

\section{Table 2.2}

\begin{tabular}{|c|c|c|c|}
\hline Variable & Categories & $\mathbf{n}$ & $\begin{array}{c}\text { Percent }(\%) / \\
\text { Mean } \pm \text { SD }\end{array}$ \\
\hline \multirow[t]{2}{*}{ Gender } & Female & 65 & 73 \\
\hline & Male & 24 & 27 \\
\hline \multirow[t]{2}{*}{ Marital Status } & Not Currently Married & 27 & 30.3 \\
\hline & Currently Married & 56 & 62.9 \\
\hline \multirow[t]{2}{*}{ Education } & High School or GED & 23 & 25.8 \\
\hline & College Degree or Higher & 62 & 69.7 \\
\hline \multirow[t]{2}{*}{ Income } & $<=\$ 50,000$ & 40 & 44.9 \\
\hline & $>=\$ 50,000$ & 27 & 30.3 \\
\hline Diabetes & Prediabetes & 34 & 38.2 \\
\hline Status & Diabetes & 55 & 61.8 \\
\hline Age & Years & 89 & $58.5 \pm .260$ \\
\hline Household & $\#$ & 66 & $1.24 \pm 1.11$ \\
\hline \multicolumn{4}{|l|}{ Size } \\
\hline Program & $\%$ & 89 & $60.8 \pm 27.6$ \\
\hline \multicolumn{4}{|l|}{ Attendance } \\
\hline Percent Body & $\%$ & 72 & $-1.74 \pm 5.08$ \\
\hline \multicolumn{4}{|l|}{ Weight } \\
\hline Benefits & Mean Score & 70 & $5.45 \pm .485$ \\
\hline Barriers & Mean Score & 70 & $4.31 \pm .924$ \\
\hline
\end{tabular}




\subsubsection{Initial Analyses}

Bivariate correlations showed significant associations for some demographic variables and main variables of interest (Appendix D). Specifically, income $(r=-.266 ; p=.029)$ and household size $(r=-.248 ; \mathrm{p}=.044)$ had a weak negative relationship with benefits. For barriers, there was a weak positive relationship with age $(\mathrm{r}=.275 ; \mathrm{p}=.021)$, but a moderate positive relationship with program attendance $(\mathrm{r}=-.325 ; \mathrm{p}=.029)$. Likewise, percent body weight had a weak, negative relationship with program attendance $(\mathrm{r}=-.251 ; \mathrm{p}=.033)$. As described in our prior publication, principal component analysis (PCA) was used to test the reliability of the benefits and barriers measure (Shawley-Brzoska \& Misra 2018). Two components loaded of importance: benefits and barriers (Shawley-Brzoska \& Misra, 2018). The mean benefits and barriers scores indicated a very high level of satisfaction with the DPM program, a high degree of benefits (mean $=5.45 \pm .485)$ and low barriers (mean=4.31 \pm .924$)$ (Table 2.2). In other words, a majority of individuals were satisfied with the program and with the changes to their personal lifestyle behaviors as a result of program participation.

\subsubsection{Benefits Models}

All model results for benefits are displayed in Table 2.3 and figure displayed in Appendix E. Model 1 was not significant, $\left(F(1,68)=1.405, p=.240, R^{2}=.020\right)$, which indicates that there is no direct relationship between benefits and percent body weight. Model 2 was marginally significant $\left(\mathrm{F}(1,68)=3.862, \mathrm{p}=.053, \mathrm{R}^{2}=.054\right)$, suggesting that there is some relationship between program attendance and benefits. Model 3 was significant $(\mathrm{F}(1,70)=4.723, \mathrm{p}=.033$, $\mathrm{R}^{2}=.063$ ), with an association between percent body weight and program attendance. The final model for benefits, Model 4, was not significant $\left(F(2,67)=2.210, p=.118, R^{2}=.062\right)$, for relationship between program attendance on benefits and percent body weight. The standardized parameter estimate $(B=.232)$ reveals a positive direct relationship but a marginal effect (not statistically significant) between program attendance and benefits. In other words, for every percent increase in program attendance, the percent body weight decreased by an average of 067 point ( $\mathrm{SE}=.031 ; \mathrm{p}=.033$ ). Additionally, $5 \%$ of the variance in percent body weight was explained by program attendance.

Table 2.3

\begin{tabular}{|c|c|c|c|c|c|c|c|c|c|c|}
\hline \multicolumn{11}{|c|}{ Benefits Models } \\
\hline & & $\mathbf{F}$ & df & $\mathbf{R}^{2}$ & $\begin{array}{c}\text { Adj. } \\
\mathbf{R}^{2}\end{array}$ & B & SE B & $B$ & $t$ & p-value \\
\hline \multirow{2}{*}{$\begin{array}{l}\text { Model 1* } \\
\text { (Percent Body } \\
\text { Weight) }\end{array}$} & Constant & & & & & 6.325 & 6.917 & & .914 & .364 \\
\hline & Benefits & 1.405 & 1,68 & .020 & .006 & -1.498 & 1.264 & -.142 & -1.185 & .240 \\
\hline \multirow{2}{*}{$\begin{array}{l}\text { Model 2* } \\
\text { (Program } \\
\text { Attendance) } \\
\end{array}$} & Constant & & & & & 25.649 & 23.684 & & 1.083 & .283 \\
\hline & Benefits & 3.862 & 1,68 & .054 & .040 & 8.506 & 4.328 & .232 & 1.965 & .053 \\
\hline \multirow{2}{*}{$\begin{array}{l}\text { Model 3* } \\
\text { (Percent Body } \\
\text { Weight) }\end{array}$} & Constant & & & & & 2.996 & 2.255 & & 1.329 & .188 \\
\hline & $\begin{array}{c}\text { Program } \\
\text { Attendance }\end{array}$ & 4.723 & 1,70 & .063 & .050 & -.067 & .031 & -.251 & -2.173 & $.033 * *$ \\
\hline Model 4* & Model & 2.210 & 2,67 & .062 & .034 & & & & & .118 \\
\hline
\end{tabular}




\begin{tabular}{lllllll}
\hline \multirow{2}{*}{$\begin{array}{l}\text { Percent Body } \\
\text { Weight) }\end{array}$} & Constant & 7.870 & 6.877 & & 1.144 & .257 \\
\cline { 2 - 7 } & Program & -.060 & .035 & -.210 & -1.725 & .089 \\
& Attendance & -.986 & 1.281 & -.094 & -.770 & .444 \\
\cline { 2 - 7 } & Benefits & & & & & \\
\hline
\end{tabular}

$*$ Covariates for models= age, education, diabetes status, income, marital status household size and gender

$* *$ Significant, $\mathrm{p}<0.05$

\subsubsection{Barriers Models}

All model results for barriers are displayed in Table 2.4. Model 1 was not significant, ( $F$ $\left.(1,68)=.746, \mathrm{p}=.391, \mathrm{R}^{2}=.011\right)$, which indicates that there is no direct relationship between barriers and percent body weight. Model 2 was significant $\left(F(1,68)=17.669, p=.000, R^{2}=.206\right)$, suggesting that there is a relationship between program attendance and barriers. The standardized parameter estimate $(B=.454)$ reveals a positive direct relationship and suggests that there is a significant association between program attendance and barriers. For every additional one-point increase in barriers (higher scores=less barriers), increases the program attendance by an average of 8.76 percent $(\mathrm{SE}=2.083 ; \mathrm{p}=.000)$. Additionally, $2 \%$ of the variance in program attendance is explained by barriers. Model 3 was significant $\left(\mathrm{F}(1,70)=4.723, \mathrm{p}=.033, \mathrm{R}^{2}=.063\right)$, with an association between percent body weight and program attendance. The parameter estimate $(B=-$ .251) reveals a negative direct relationship. There was a significant association between percent body weight change and program attendance. More specifically, for every additional percent increase in program attendance, the percent body weight reduced by an .067 percent $(\mathrm{SE}=.031$; $\mathrm{p}=.033$ ). Additionally, $6 \%$ of the variance in percent body weight is explained by program attendance. The final model for barriers, i.e., Model 4, was significant $(F(1,67)=4.089, p=.021$, $\mathrm{R}^{2}=.109$ ), for relationship between program attendance on barriers and percent body weight. For every additional one-point increase in barriers (higher scores=less barriers), increases the percent body weight by an average of 1.46 percent $(\mathrm{SE}=.717 ; \mathrm{p}=.046)$ when controlling for program attendance.

Table 2.4

\begin{tabular}{|c|c|c|c|c|c|c|c|c|c|c|}
\hline \multicolumn{11}{|c|}{ Barriers Models } \\
\hline & & $\mathbf{F}$ & df & $\mathbf{R}^{2}$ & $\begin{array}{c}\text { Adj. } \\
\mathbf{R}^{2}\end{array}$ & B & SE B & B & $t$ & p-value \\
\hline Model 1* & Constant & & & & & -4.324 & 2.941 & & -1.471 & .146 \\
\hline $\begin{array}{l}\text { (Percent Body } \\
\text { Weight) }\end{array}$ & Barriers & .746 & 1,68 & .011 & -.004 & .576 & .668 & .104 & .863 & .391 \\
\hline Model 2* & Constant & & & & & 34.280 & 9.178 & & 3.735 & $.000 * *$ \\
\hline $\begin{array}{l}\text { (Program } \\
\text { Attendance) } \\
\end{array}$ & Barriers & 17.669 & 1,68 & .206 & .195 & 8.758 & 2.083 & .454 & 4.203 & $.000 * *$ \\
\hline Model 3* & Constant & & & & & 2.996 & 2.255 & & 1.329 & .188 \\
\hline (Percent Body & Program & 4.723 & 1,70 & .063 & .050 & -.067 & .031 & -.251 & -2.173 & $.033 * *$ \\
\hline Weight) & Attendance & & & & & & & & & \\
\hline
\end{tabular}




\begin{tabular}{lccccccccc}
\hline $\begin{array}{l}\text { Model 4* } \\
\text { (Percent Body } \\
\text { Weight) }\end{array}$ & Model & 4.089 & 2,67 & .109 & .082 & & & & $.021^{* *}$ \\
\cline { 2 - 8 } & Constant & & & -.868 & 3.087 & & -.281 & .779 \\
\cline { 2 - 8 } & $\begin{array}{c}\text { Program } \\
\text { Attendance }\end{array}$ & & -.101 & .037 & -.351 & -2.713 & $.008^{* *}$ \\
\cline { 2 - 8 } & Barriers & & & & & & & & \\
\hline
\end{tabular}

*Covariates for models $=$ age, education, diabetes status, income, marital status, household size and gender

$* *$ Significant, $\mathrm{p}<0.05$

\subsection{Discussion}

The results of this study suggested that barriers (and not benefits) are directly related to decrease in percent body weight, but only after controlling for program attendance. The positive relationship between barriers and program attendance suggests that barriers such as health status, work and family activities increased attendance of the DPM program sessions. Therefore, barriers did impact the success in program participation. There was also a trend towards greater benefits leading to a better program attendance. However, further studies should explore this relationship to confirm this marginal effect. These results suggest that this study fails to support only the mediation hypotheses.

The findings of this study showed that participants' experience in a comprehensive lifestyle intervention was positive. Overall, participants expressed programs sessions were informative and provided useful knowledge and skills for successful lifestyle changes. They also valued their interactions with HCs, program leaders and educational session leaders to motivated them for active participation and lifestyle behavior changes. Further, participants identified few deterrents for program engagements. Hence, this study presents interesting findings related to program engagement and supports previous research on program satisfaction of lifestyle interventions in various populations (French et al., 1998; Venditti et al., 2014; Caro-Bautista et al., 2014). Our findings are helpful when designing and adjusting lifestyle interventions to address program benefits and barriers for successful programs. Prior diabetes programs that have identified successes or potential improvements related to personal and programmatic issues have shown promising health outcomes (Straus, Tetroe, \& Graham, 2013; Tripp-Reimer \& Doebbeling, 2004; Biedenweg et al., 2014)

Unlike prior studies, participant demographic characteristics such as age, gender or socioeconomic status were not associated in the model and did not predict percent body weight. One plausible explanation might be the homogenous sample where participants were a group of mostly college-educated individuals with reasonable income compared to the rest of the WV population. Limited variance with both education and income levels could explain why there was no association with percent body weight change at the end of the program. Likewise, a significant relationship was found for benefits in the bivariate relationship but was not found significant with program attendance and percent body weight; this could be due to the lack of variability between responses. Participants were overwhelmingly positive in their perception of the DPM benefits and hence there was a skew towards participants" responses of "strongly agree." to various questions. Perhaps the program benefits were related to higher program attendance and low attrition as participants were excited with their readiness to change and health behavior modifications (Ali, Echouffo-Tcheugui \& Williamson, 2012). Program attendance was also related to weight loss as suggested in other lifestyle programs (Ali, 
Echouffo-Tcheugui \& Williamson, 2012; Jeffery, et al., 1978; Kramer et al., 1986). Consequently, overall program benefits could have impacted the participant's responses to barriers to program participation in this Appalachian region.

Benefits of the DPM program were related to having a health coach, program materials, lifestyle modification, program results, health provider, family/friends, diabetes risk and overall satisfaction. Barriers included items related to transportation, weather, health status, work and family activities. Higher mean scores were presented for benefits in comparison to barriers. However, only the barriers component was significant with percent body weight in the final model. This showed that the barriers have more of an impact on percent body weight than program benefits. It is known that if a person perceives high benefits they are most likely to have low barriers (Shawley-Brzoska \& Misra, 2018; Thomson, Buckley, \& Brinkworth, 2016). Ideally, this would result in high program success and lower attrition rate. More specifically, participants with higher barrier scores (lower barriers) were found to be significant with percent body weight when controlling for program attendance. Therefore, DPM participants who had more barriers resulted in better program attendance, which led to change in percent body weight. Additionally, better program attendance resulted in reduction in percent body weight.

Behavioral, psychological and environmental factors suggested by health behavior theories can explain or predict a specific behavioral change (Glanz, Rimer, \& Viswanath, 2008). A behavior or lifestyle modification can be caused by an individual's initial perception. Whether it is positive or negative, the individual tends to choose the behavior that results in the most benefits (Sharifirad, Azabakht, Feizi, Kargar, \& Mohebi, 2013). Most individual behaviors are dependent on results of the action, which could be lead to benefits or barriers. Benefits and barriers can relate to personal experience and level of performance with a behavior (McEvoy \& Nathan, 2007). Individuals' behaviors tend to be influenced by prior experience and knowledge (Pender, Murdaugh, \& Parsons, 2002).

There were several strengths of this study. This study used the Baron and Kenny method, which is one of the most widely techniques for testing mediation. In addition, this study is one of few studies that examined benefits and barriers of a diabetes prevention and management program with both individuals of prediabetes and diabetes. Speculations about program success outside of required metrics has been of interest, but still little is known about how benefits and barriers can play a role in program success or failure. Addressing benefits and barriers could be an important predictor in success of diabetes lifestyle programs. Based on recruitment and eligibility of the program, the study sample was relatively generalizable in sex, marital status, and diabetes status. Additionally, diabetes status was based originally on a self-report measure, but it was confirmed with an A1c value. With the inclusion of both diabetes statuses (prediabetes and diabetes), multiple sites and a wide range of age groups, study findings could be generalizable to WV and beyond.

The results should be considered in context to the following limitations. With using the Baron and Kenny method, concerns with statistical power were taken into consideration. Additionally, study variables such as demographic characteristics and survey questions were self-report, which could have led to measurement error and misclassification bias. Specifically, determination of benefits and barriers scores were based on a self-report measure that was developed for this study. Even with the PCA analysis, the survey has not been implemented with other audiences or studies. In addition, sample size was small and there were issues with 
missingness in the data. For this study, the total sample had 89 participants while the final model only included 70. Moreover, social desirability bias may have influenced responses on demographics and lifestyle questions. In addition, weight loss is a simplistic way of observing weight changes. Some individuals might have improved muscle mass and reduced percent body fat with no changes in weight or weight loss.

\subsection{Conclusion}

These findings clearly indicated that while participants had positive perceptions of the DPM program, minimal barriers to program participation improved their attendance. Program benefits may have improved session attendance for knowledge on lifestyle modifications such as healthy dietary habits, physical activity, adherence and coping with their chronic conditions. Furthermore, quantitative approaches (such as surveys) along with program measures (such as weight and attendance) can be used to thoroughly conceptualize and comprehend the program effectiveness and feasibility of lifestyle programs. Examining these specific factors in a diabetes program can also allow to culturally tailor future diabetes programs in WV.

There exists a gap between knowledge, risk factors and lifestyle modifications to effectively lower complications, morbidity and mortality for individuals with chronic conditions. Hence, identifying participants' benefits and barriers of the program to improve program attendance for improved health outcomes can be done in future studies and fill a gap in lifestyle intervention research. Plans for future research could include the following: replicating the benefits and barriers survey in diverse population to establish validity and reliability of the scale, and ensure consistency of the measure, exploring the benefits and barriers are identified during process evaluation to improve program implementation and sharing the findings on identified benefits and barriers. Lastly, future studies should expand the effectiveness of lifestyle interventions for participants with chronic conditions. 


\section{CHAPTER 3}

Assessing social support and program outcomes in a diabetes prevention and management program 


\subsection{Significance}

Social Support is defined in various ways (depending on the context or situation) (Hupcey, 1998) and described as assistance or trust received from individuals, groups and the larger community through social ties that provide psychological and material resources. Social support may positively or negatively impact lifestyle or personal well-being of recipients (Hunt, Grant \& Pritchard, 2012). It can involve helpful information, encouragement, financial incentive, equipment, listening, and assessment (Birch, 1998). In addition, social support can be the motivating factor for positive health behaviors, which can play a role in maintaining and promoting good health (Reblin \& Uchino, 2008). Research shows individuals begin or continue health behaviors based on their level of social support (Birch, 1998; Uchino, Cacioppo, Kiecolt-Glaser, 1996). Hupcey (1998) indicated that social support is a multi-faceted concept that has been difficult to conceptualize, define and measure and theoreticians and researchers lack consensus on its theoretical and operational definition. In addition, in the past few decades, models of social support have included different kinds of social interaction (Hupcey, 1998). Currently, social support in behavioral research encompasses perceptions, behaviors, relationships, social systems, quality of support and interactions. Social support is a complex and multidimensional construct; there are different types of social support such as emotional support (defined as the care or sympathy toward another person), information support (being a source of knowledge), material support (providing a tangible resource), and appraisal support (providing feedback, praise or suggestions) (Birch, 1998; Uchino et al., 1996). However, there are two broad categories of the social support (i.e. support from family and support from friends) that have important implications to help guide participants of the DPM program.

The theoretical foundations of social support are based on the Social Comparison Theory, Social Exchange Theory and Social Competence (Langford, Bowsher, Maloney, \& Lillis, 1997). The Social Comparison Theory compares the availability of social support to patients and others as related to their disease and for motivation and behavior changes, while the Social Exchange Theory demonstrates intangible and metaphorical costs as well as benefits to those who engage in and provide support for mutually rewarding activities that explains life satisfaction, the ability to receive social support and how behaviors are consistent with goals (Stevens, 1992). Social competence, on the other hand, is the ability to effectively interact with the environment and/or individuals and groups while learning from past experiences/behaviors and others to manage their chronic conditions over time (Taborsky \& Oliveria, 2012).

Social support can be measured in terms of structural support or functional support (Barrera, 1986). Structural support (also called social integration) refers to the extent to which a recipient is connected within a social network, like the number of social ties or how integrated a person is within his or her social network (Barrera, 1986; Willis, 1991). Family relationships, friends, and membership in clubs and organizations contribute to social integration. Functional support looks at the specific functions that a member in the social network can provide, such as the emotional, instrumental, informational, and companionship support (Uchino, 2004). Research has shown that emotional support may play a more significant role in protecting individuals from the deleterious effects of stress than structural means of support, such as social involvement or activity (Kessler \& McLeod, 1984). In term of diabetes self-management, the absence of support from significant others (structural) and lack of individual characteristics (functional) can be associated with poor compliance to medial regimens, lifestyle behaviors and disease selfmanagement (Beets, Cardinal \& Alderman, 2010; Shier, Ginsburg, Howell, Volland \& Golden, 2013; Verheijden, Bakx, Van Weel, Koelen, \& Van Staveren, 2005). 
Diabetes is a chronic condition that requires extensive lifestyle modification and selfmanagement for optimal glycemic control over time (Osborn et al., 2010). Yet, these lifestyle changes and adherence to complex self-management practices (such as diet, physical activity, blood glucose monitoring and medication) are often difficult to implement and maintain; social support is viewed as an important influential factor for their ongoing disease self-management (Rad, Bakht, Feizi \& Mohebi, 2013). The absence of social support is often found to be associated with unhealthy lifestyle such as poor diet, smoking and sedentary lifestyle (Cohen, Gottlieb, \& Underwood, 2000). Both structural and functional support can be beneficial to promote diabetes education, encouraging and enabling positive behavioral changes and regimen adherence, or it can also be constraining or negative such as non-reciprocal, stressful or over-demanding support, social interactions or relationships (Beets et al., 2010; Shier et al., 2013; Verheijden et al., 2005). Hence, the complex relationship between social support and diabetes self-management can pose challenges to translate, identify and capitalize the beneficial and positive aspects of social support and minimize the negative aspects for optimal self-management of diabetes. Specifically, chronic diseases can indicate higher risk based on an individual's social networks; for example, the likelihood of becoming obese can increase if an individual in their social support network became obese (Bishop et al., 2013). Furthermore, individuals relate better with their romantic partners, family and friends if they have the same disease status (Embuldeniya et al., 2013). Thus, previous studies that focused on lifestyle interventions and weight loss programs have incorporated the different levels of social support as mediating or moderating factors in their analysis (Piette et al., 2013; Shah et al., 2015; Marquez et al., 2016; Heard et al., 2001). Results from weight loss programs show individuals are more likely to lose weight if they had frequent family and friend support (Sallis \& Owen, 1999; Trost et al., 2002; Kamphuis et al., 2006; Shaikh et al., 2008; Wang et al., 2015). Furthermore, Wing et al. found that the weight loss success of the social contacts outweighed the number of social contacts for participants (2006). Therefore, the success of a few close social contacts was considered more important than the number of friends or family enrolled in the program with them.

Perceived social support has shown to have both buffering and direct effects on mental health and linked to adaptive experiences (Lakey \& Cronin, 2008). Perceived social support and adherence to chronic disease regimens is positively associated with social support, leading to better health outcomes such as glycemic control and hospitalization risk (Rosland et al., 2008). Specifically, social support from family and friends can positively impact self-monitoring of blood glucose and diabetes meal planning (Gallant, 2003). The effect of social support has also been examined in diabetes programs, such as the Diabetes Study of Northern California (DISTANCE). It examined associations between social support and diabetes self-management behaviors and found that higher social support resulted in improved lifestyle behavior modifications (Rosland et al., 2008 \& 2014). However, the importance of social support declined as diabetes selfmanagement from lifestyle changes moved to more skilled behaviors such as better glucose monitoring and medication adherence (Osborn et al., 2010; Van Den Arend et al., 2000).

For diabetes education programs that focus on lifestyle modifications, clinical outcomes (e.g., glycosylated hemoglobin level, cholesterol etc.) are often tracked and monitored for the participants in order to assess the long-term program effectiveness as a result of lifestyle modifications and sustainability of the healthy behavior changes (Mayo Clinic, 2018). Specifically, clinical values such as glycosylated hemoglobin level (or A1c) and lipid profile (such as triglycerides, total cholesterol, LDL cholesterol and HDL cholesterol) can be improved 
by lifestyle changes. However, specific health behaviors are associated with different clinical parameters. For example, high levels of LDL or the "bad" cholesterol can be improved by making dietary changes such as restriction of fat/saturated fat/cholesterol in the diet and increasing high fiber intake (Mayo Clinic, 2018; National Heart, Lung, and Blood Institute, 2016; American Heart Association (AHA), 2016; CDC, 2016). Research supports that simply avoiding food with trans fats and fried foods could lower serum LDL cholesterol. Likewise, HDL levels or the "good" cholesterol can be improved by increasing physical activity to as little as 60 minutes of moderate exercise a week (AHA, 2016; CDC, 2017; Rosenson, 2016; Mayo Clinic, 2018). Additionally, A1c can be improved with both lifestyle modifications (exercise and diet). Thus, lifestyle behaviors can play a significant role in combating diabetes prevention and control. Hence, this study assessed the impact of social support on health-related behaviors (diet and physical activity) and clinical outcomes (HDL cholesterol, LDL cholesterol, and A1c).

Another study, the Healthy Living Partnerships to Prevent Diabetes (HELP PD), examined how program involvement influenced their social support persons (SSPs) (individuals in their social networks) (Bishop et al., 2013). Participants' SSPs were positively influenced by program participation regarding weight loss and changes in eating habits. Likewise, Hwang and his colleagues also examined structural support in an online weight loss program and found that social support can influence weight loss behaviors (2011). In another study, Hunt and his colleagues examined self-efficacy, social support and social problem solving on the impact on diabetes selfmanagement (2012). Results showed a positive association between these variables; the authors concluded that diabetes programs will be benefited if they incorporate self-efficacy, social support and social problem solving during implementation.

While attention has focused on individuals with diabetes, their families and, sometimes, communities, the broader social context of social support is often overlooked for diabetes selfmanagement (Wiebe, Helgeson \& Berg, 2016). For example, culture, while often viewed from within a family or individual belief system, is also embedded within the larger society (Wallhagen \& Lacson, 1999). Cultural tailoring of diabetes programs to the Appalachian cultures and rural contexts such as WV, can improve effectiveness and sustainability. Furthermore, it can have an impact on individual's social support and quality of life (Kishore, Nagaraj \& Ravouru, 2013; Kishore et al., 2013). Research shows rural families are more likely to change lifestyle behaviors (dietary habits or engaging in exercise) when they were supported by family and friends (Pullen, Walker \& Fiandt, 2001; Lina Ma et al., 2015; Wewers et al., 2006). By recognizing the culture and context of populations, researchers can begin to understand the relevance of culture in diabetes prevention and self-management and everyday experience as related to nutrition, physical activity, and other behaviors. Hence, an examination of social support in the broader context for diabetes programs is essential for improved adoption and sustainability in rural Appalachian communities.

Despite a surge of research that examined social support in regards to lifestyle programs, there is a dearth of social support assessment in Appalachia. In addition, most studies have measured and scored the level of social support and related it with various outcome measures such as HbA1c, weight, BMI, blood lipids, diet adherence, physical activity quality of life, blood pressure, support satisfaction and diabetes knowledge (Van Dam et al., 2000). Results have consistently shown that social support, assessed among diverse populations, has a positive impact on glycemic control, self-management behaviors, and quality of life (Osborn et al., 2010; Van Den Arend et al., 2000). 
Positive relationships between social support and diabetes self-management outcomes have been established, yet, there is very little research that has been done with individuals with pre-diabetes in lifestyle programs, such as the DPP, modified DPP or a combined diabetes prevention and management program. Moreover, social capital, the associated social support networks or social relationships and social linkages in WV may be unique and/or different than urban and other areas in the country (Welch, 2011). The culture of WV/Appalachian region regarding disease conditions is fatalistic and dependent on other individuals' perceptions of their health (Welch, 2011; Royse \& Dignan, 2011; Hag Hamed, 2017). Social support is frequently received from family and friends but can also be received from their peers in lifestyle programs. In addition, peer educators and lay health workers (such as the CHWs) that serve as lifestyle coaches have shown to provide social support to individuals (Katula et al., 2011; Alaofe et al., 2017). Thus, effective programs can be tailored to the communities and participants based on their available social networks.

The DPM program used lifestyle coaches who were recruited from WV communities to serve as HCs for the program. These HCs had prior community engagement in the community and were familiar with the culture of the region (details in 2.2). They provided one-on-one engagement during their weekly communication with the participants. For the DPM program, the HCs provided direct and indirect support for lifestyle behavior changes through education, weekly communication, feedback on participant's weekly diet logs and physical activity (step count using pedometers). However, perceived social support by participants from HCs, based on weekly communications and in the educational group sessions is unknown. Therefore, the aim of this study was to examine participant's perceived social support and its association with program attendance and clinical outcomes (HDL cholesterol, LDL cholesterol and A1c).

\subsection{Methods}

Using pre- and post-program data, this analysis examined DPM participants' perceived social support and clinical outcomes. The primary aim of the DPM program was to evaluate the feasibility and effectiveness of the program. Data was collected at the baseline, mid-program (at 6 months) and post-program (at 12 months). As mentioned in Chapter 1, anthropometrics, bloodwork and survey data were collected during the assessments.

Research Question 1: Did social support for physical activity have an impact on program outcomes such as attendance, HDL and A1c?

Research Question 2: Did social support for dietary modification have an impact on program outcomes such as attendance, LDL and A1c?

Since physical activity is positively associated with HDL and poor diet is negatively associated with LDL, we explored the relationship of PA social support with HDL and diet social support with LDL. Two valid and reliable questionnaires were used to elicit perceived social support from participants regarding their social support from friends and family. Participants had the opportunity to rate the level of social support in conversations and/or situations involving exercise and diet. The Social Support and Exercise and Social Support and Eating Habits surveys (Sallis, Grossman, Pinski, Patterson, \& Nader, 1987) were administered to participants in the DPM program at the 6-month and 12-month blood screenings as the social support survey was approved through the IRB after the baseline blood screening. This study included the following objectives: 1) Determine the level of social support related to diet, 2) Determine the level of 
social support related to physical activity, and 3) Examine the impact of social support on program outcomes such as attendance, HDL, LDL and A1c while controlling for demographics.

More specifically, this research assessed the relationship between (1) exercise social support, HDL, attendance and A1C and (2) diet social support, attendance, LDL and A1c. Both analyses controlled for demographic variables (age, household size, gender, income, marital status, education, and diabetes status). The null hypothesis was defined by capturing no variance in exercise and/or diet social support with attendance, LDL, HDL, or A1c. The alternative hypothesis was defined as variance accounted for in attendance, LDL, HDL, and/or A1c on participant diet and/or exercise social support. To test these hypotheses, a variety of multiple linear regression statistical tests were used.

\subsubsection{Measures}

\subsubsection{Demographics}

Selected variables were chosen from the larger DPM program dataset. All participants were provided with a unique DPM program participant ID with four components (see section 3.2.1.1) that allowed anonymity but provided the investigators the ability to connect the survey with demographic information collected at baseline. Demographic questions included the following demographic information of the participant: age, household size, gender, education, marital status, diabetes status and income.

The participant's date of birth was used to calculate the age of each participant. This variable was used as a continuous variable. The number of members in their household was provided and was used as a count, ordinal variable. The categorical variable, gender, was selfidentified as either female and male. For coding purposes, females were categorized as a 0 while males will be categorized as a 1 . The categorical variable, marital status, was coded: 1) currently married and 0) not currently married. The categorical variable, education level, was coded: 0 ) high school degree/GED and1) college degree or higher. This categorical variable was used in prior DPM programs and hence used for this pilot study. The categorical variable, diabetes status, was defined as having prediabetes or diabetes based on their self-reported medical history and supplemented with A1c values (diabetes $\geq 6.5$; prediabetes 5.7 to 6.4). For coding purposes, those with diabetes were categorized as a 1 while those with prediabetes were categorized as a 0 . The categorical variable, annual income, was identified into the following groups for coding purposes: 0) less than or equal to $\$ 50,000$ 1) greater than or equal to $\$ 50,000$.

\subsubsection{Predictor and Outcome Variables}

Program attendance was computed by summing attendance for all 22 sessions and percent attendance was calculated by dividing the total sessions attended by the total number of program sessions (22) and multiplying it by 100; participants needed a minimum of four sessions for this calculation, which is based on the Diabetes Prevention Program Recognition Standards. The participant's fasting blood was drawn at the baseline, mid-program and final program blood screenings. HDL was used as a continuous variable and the change in HDL was calculated from baseline and post-program HDL results (mg/dL). LDL was used as a continuous variable and the change in LDL was calculated from baseline and post-program LDL results (mg/dL). A1c was used as a continuous variable and the change in A1c was calculated from baseline and postprogram A1c results. 


\subsubsection{Social Support Survey}

The Social Support and Exercise and Social Support and Eating Habits surveys (Sallis et al., 1987) measured social support in health-related diet and physical activity behaviors. These two social support measures for adults have been supported for scale reliability, internal consistency and validity in the literature that used test-retest and internal consistency assessments along with criterion-related validity (Sallis et al., 1987) (see Appendix G, H and I). The exercise survey consisted of 12 questions while the diet survey had 10 questions, on a Likert type response scale: 1) none, 2) rarely, 3) a few times, 4) often, 5) very often. Responses related to "does not apply" or "blanks" were considered missing data for the analysis. For the Social Support and Eating Habits Survey, the scores were summed separately for family and friends. Furthermore, an additional 5 questions were summed together for the "Encouragement" and the "Discouragement" subscales, respectively. For the Social Support and Exercise survey, the questions were scored for friends and family i.e. 6 questions were summed to form the "Family Participation subscale", 4 questions were summed for the "Friend Participation" subscale score and finally 3 questions were used to create "Family Rewards and Punishment" subscale. Participants were asked to complete the survey by themselves to reduce bias. Confidentiality was assured throughout the process, by using the DPM participant ID.

\subsubsection{Statistical Analyses}

Attendance and changes in A1c, HDL and LDL were the dependent (continuous) variables. Social support was the independent (continuous) variable and was used as the primary predictor in the multivariate models. Age was analyzed as an independent, continuous variable. Education level, income, household size, marital status and gender were analyzed as independent (categorical) variables. Prior to importing data into SPSS for analyses, the Microsoft Excel data file was examined and cleaned. Specifically, all the program data was double entered and examined for missingness. Drop rate calculated from those participants who completed baseline and post-program assessments for the DPM program. Those with missing a baseline or postprogram assessment were excluded from all analyses. Multiple linear regression examined the relationship between social support and attendance, change in A1c, change in HDL and change in LDL after controlling for age, education level, income, marital status, household size, and gender. These variables were used due to prior associations in published studies and/or theoretical framework. All categorical variables were dummy coded in the model. Group differences were assessed for the program sites (Morgantown and Charleston) and by diabetes status (prediabetes and diabetes). The regression equation listed below was used and all regression models are displayed in Table 3.1.

\subsection{Example Regression Equation}

\section{Regression Line with Covariates}

$\mathrm{A}=\mathrm{a}+\mathrm{B}_{1} \mathrm{X}_{1}+\mathrm{B}_{2} \mathrm{X}_{2}+\mathrm{B}_{3} \mathrm{X}_{3}+\mathrm{B}_{4} \mathrm{X}_{4}+\mathrm{B}_{5} \mathrm{X}_{5}+\mathrm{B}_{6} \mathrm{X}_{6}+\mathrm{B}_{7} \mathrm{X}_{7}+\mathrm{B}_{8} \mathrm{X}_{8}+\mathrm{E}$

$\underline{\text { Regression Line with only outcome and predictor }}$

$\mathrm{A}=\mathrm{a}+\mathrm{B}_{8} \mathrm{X}_{8}+\mathrm{E}$

$\mathrm{A}=$ Attendance, dependent variable, what is being predicted or explained

$\mathrm{X}_{1}=$ Age, $\mathrm{X}_{2}=$ Gender, $\mathrm{X}_{3}=$ Martial Status, $\mathrm{X}_{4}=$ Educational level, $\mathrm{X}_{5}=$ Diabetes Status, $\mathrm{X}_{6}=$ =ncome, $\mathrm{X}_{7}=$ Household size, $\mathrm{X}_{8}=$ Social Support, independent variables that is explaining the variance in 
$\mathrm{a}=$ the intercept

$\mathrm{E}=$ error term

\section{Table 3.1}

\begin{tabular}{|l|l|c|}
\hline \multicolumn{3}{|l|}{ Social Support with Eating Habits Models } \\
\hline Model & Dependent Variables & Independent Variables \\
\hline $1^{*}$ & Attendance & Encouragement-Family \\
\hline $2^{*}$ & Attendance & Encouragement-Friends \\
\hline $3^{*}$ & Attendance & Discouragement-Family \\
\hline $4^{*}$ & Attendance & Discouragement-Friends \\
\hline $5^{*}$ & LDL & Encouragement-Family \\
\hline $6^{*}$ & LDL & Encouragement-Friends \\
\hline $7^{*}$ & LDL & Discouragement-Family \\
\hline $8^{*}$ & LDL & Discouragement-Friends \\
\hline $9^{*}$ & A1c & Encouragement-Family \\
\hline $10^{*}$ & A1c & Encouragement-Friends \\
\hline $11^{*}$ & A1c & Discouragement-Family \\
\hline $12^{*}$ & A1c & Discouragement-Friends \\
\hline Social Support with Exercise Models & \\
\hline Model & Dependent Variables & Independent Variables \\
\hline $13^{*}$ & Attendance & Family Participation \\
\hline $14^{*}$ & Attendance & Friend Participation \\
\hline $15^{*}$ & HDL & Family Participation \\
\hline $16^{*}$ & HDL & Friend Participation \\
\hline $17^{*}$ & A1c & Family Participation \\
\hline $18^{*}$ & A1c & Friend Participation \\
\hline
\end{tabular}

*Covariates for all models= age, education, diabetes status, income, marital status household size and gender

\subsection{Regression Assumptions}

SPSS was used to conduct all analyses; p-value was set to 0.05 level of significance. Sample size appropriateness was determined by making sure that there were at least 20 cases for each independent variable. Correct model specification was conducted by examining the bivariate relationship between each of the dependent variables (attendance rate, A1c, HDL and LDL) and the independent variables (social support, age, education, income, marital status, household size and gender). Spearman correlations and associated significance level was used to determine the relationship of variables. Based on theoretical knowledge, all relevant variables were included. If any of the independent or control variables were not significant (i.e. $p>0.05$ ), they were excluded from further analyses.

Linearity was examined using scatter plots with a regression line for every significant predictor and the outcome variable. Homoscedasticity was determined if there was a consistent residual spread around zero, which was the constant variance of residuals. Predicted values of the independent variables were compared to the residuals. To determine multicollinearity, bivariate relationships were examined using the strength of the correlation and significance level. A high correlation among predictor variables indicated multicollinearity and posed a problem due the 
increase of standard errors and related decrease in the likelihood of finding statistical significance. In addition to this, variance inflation and tolerance was analyzed to better check for multicollinearity. Variance inflation values was considered appropriate when they were less than 10 and close to two; tolerance values close to one were considered adequate. Descriptive statistics (i.e. mean, median, mode, range, skewness, and kurtosis) were assessed. A Q-Q plot and histogram was used to determine normality of residuals. The normal distribution was also determined by examining the mean, mode, skewness, and kurtosis of each of the variables. Detection of outliers included tests that examined leverage, discrepancy, and influence. Removing outliers from the data only occurred when the same outlier was identified in leverage, discrepancy, and influence.

\subsection{Models}

Eighteen regression models were analyzed to determine the variance accounted for by social support. Models controlled for the independent variables and included the outcome variable, attendance rate, A1c, HDL, and LDL and the independent variables except for the predictor variable, social support. Using OLS multiple regression tests, standardized coefficients/beta, adjusted R-square, and p-values were assessed for statistical significance (pvalue $<0.05$ ). To examine variance in the outcome variable, the individual predictors and the adjusted R-squared was analyzed. The R-squared value was used to determine whether social support was a good predictor of variance in the outcome variables, attendance, A1c, HDL and LDL.

\subsection{Results}

\subsubsection{Study Sample Characteristics}

Table 3.2 presents descriptive statistics on the total sample, which included 70 participants. Participants were primarily female (74\%) and ranged in age from 20 to 83 years (mean=59.01 years, $\mathrm{SD}=0.26$ ). The majority of the participants had a college degree or higher (71.4\%) and an average personal income of $<\$ 50,000(46 \%)$. Most participants were currently married $(60 \%)$ and lived in a family with a mean household size of $1.2(\mathrm{SD}=1.12)$. Sixty-one percent of individuals had diabetes and $39 \%$ had prediabetes. No differences in demographic characteristics were found between the two program sites or by diabetes status.

\section{Table 3.2}

\begin{tabular}{|lccc|}
\hline Variable & Categories & n & $\begin{array}{c}\text { Percent (\%)/ } \\
\text { Mean } \pm \text { SD }\end{array}$ \\
Gender & Female & 52 & 74.3 \\
Marital Status & Male & 18 & 25.7 \\
& Not Currently & 24 & 34.3 \\
& Married & & 60 \\
& Currently & 42 & \\
Education & Married & & 25.7 \\
& High School & 18 & \\
\hline
\end{tabular}




\begin{tabular}{|lccc|}
\hline & College & 50 & 71.4 \\
& $\begin{array}{c}\text { Degree or } \\
\text { Higher }\end{array}$ & & \\
Income & $<=\$ 50,000$ & 32 & 45.7 \\
& $>=\$ 50,000$ & 25 & 35.6 \\
Diabetes Status & Prediabetes & 27 & 38.6 \\
& Diabetes & 43 & 61.4 \\
Age & Years & 70 & $59.01 \pm 13.1$ \\
Household Size & $\#$ & 55 & $1.2 \pm 1.12$ \\
Program Attendance & $\%$ & 70 & $64.94 \pm 25.5$ \\
A1c Change & $\%$ & 43 & $-.042 \pm .479$ \\
HDL Cholesterol Change & $\mathrm{mg} / \mathrm{dL}$ & 43 & $.977 \pm 11.66$ \\
LDL Cholesterol Change & $\mathrm{mg} / \mathrm{dL}$ & 40 & $-1.16 \pm 15.10$ \\
\hline
\end{tabular}

\subsubsection{Outcome Variables}

Descriptive information for the outcome variables are presented in Table 3.2. For the outcome variable (percent attendance), an average attendance of 65 percent was found $(\mathrm{SD}=25.5)$. For the clinical outcome variables (i.e., change in LDL, HDL and A1c), baseline, $12-$ month and change in baseline to post-program values were calculated. The following results were found for A1c: 1) Baseline (mean=6.88 \pm 1.37$), 2$ ) 12-Month (mean=6.69 \pm 1.26 ), and 3) Percent Change (mean=-.042 \pm 479$)$. For HDL, the following values were baseline (mean=47.6 \pm 15.03$), 12$-Month $($ mean $=51.0 \pm 15.13)$, and Change $($ mean=.977 \pm 11.66$)$. In addition, LDL results were at baseline (mean=98.4 \pm 37.2$)$, 12-months (mean=100.6 \pm 40.94$)$ and change from pre- and post-program (mean=-1.16 \pm 15.10$)$.

\subsubsection{Initial Analyses and Social Support Survey}

Bivariate correlations showed significant associations for some demographic variables and main variables of interest (Appendix J). Specifically, gender $(r=-.336 ; p=.042)$ and education $(\mathrm{r}=-.411 ; \mathrm{p}=.011)$ had a moderate, negative relationship with LDL Change. For program attendance, there was a moderate, positive relationship with age $(\mathrm{r}=.323 ; \mathrm{p}=.006)$, but a moderate, negative relationship with encouragement with friends $(r=-.342 ; \mathrm{p}=.008)$.

The social support surveys confirmed reliability with the following Cronbach's alpha values: 1$)$ eating habits-family $(\alpha=.908), 2)$ eating habits-friends $(\alpha=.938), 3)$ exercise-family $(\alpha=.974)$ and 4$)$ exercise-friends $(\alpha=.979)$. The social support variables were analyzed separately by family and friends; descriptive information is provided in Table 3.3. Encouragement for healthy eating habits was greater by family (mean score $=10.74 \pm 5.52$ ) as compared to encouragement (eating habits) by friends (mean=8.9 \pm 4.09$)$. Conversely, for discouragement (eating habits) by family and friends, the mean score was: 1) Family- 10.5 \pm 4.86 and 2) Friends$8.80 \pm 4.41$. The social support for participation in exercise, specifically, from family and friends showed the mean value for family was $17.0(\mathrm{SD}=9.13)$ and friends was $15.3(\mathrm{SD}=7.64)$ respectively. These social support scores indicated that, overall, participants perceived a very high level of social support for exercise or physical activity, while they perceived a relatively low level of social support for their healthy eating habits. 
Table 3.3

\begin{tabular}{lllc}
\hline Survey & Variable & $\mathbf{n}$ & Mean \pm SD \\
Eating Habits & Encouragement-Friends & 62 & $10.74 \pm 5.52$ \\
Survey & Encouragement-Family & 60 & $8.9 \pm 4.09$ \\
& Discouragement-Family & 62 & $10.5 \pm 4.86$ \\
& Discouragement-Friends & 61 & $8.80 \pm 4.41$ \\
Exercise & Family Participation & 62 & $17.0 \pm 9.13$ \\
Survey* & Friend Participation & 58 & $15.3 \pm 7.64$ \\
\hline
\end{tabular}

*Family Rewards and Punishment subscale was excluded based on original PCA analysis by Sallis et al.

\subsubsection{Model Analyses}

Results for all eighteen models are displayed in table 3.4. Only one model was significant i.e., Model 2, $\mathrm{F}(1,58)=7.663, \mathrm{p}=.008, \mathrm{R}^{2}=.117$ ) with a positive (significant) association between the behavioral outcome variable (percent attendance) and social support $(\mathrm{B}=-.342$; $\mathrm{p}=.008)$ (see Table 3.4). The parameter estimate $(\mathrm{B}=-.342)$ reveals a negative direct relationship and suggested that lower levels of social support was associated with higher attendance of program sessions. For every additional one-point increase in Encouragement with Friends subscale, program attendance decreased by 1.7 percent $(\mathrm{SE}=.613 ; \mathrm{p}=.008)$. In addition, $11 \%$ of the variation in program attendance was explained by the social support provided through the encouragement by friends.

Table 3.4

\begin{tabular}{|c|c|c|c|c|c|c|c|c|c|c|}
\hline \multicolumn{11}{|c|}{ Social Support Eating Habits Models } \\
\hline & & $\mathbf{F}$ & df & $\mathbf{R}^{2}$ & Adj. $\mathbf{R}^{2}$ & $\mathbf{B}$ & SE B & $\bar{B}$ & $t$ & p-value \\
\hline Model 1* & Constant & & & & & 75.609 & 5.65 & & 13.382 & .000 \\
\hline $\begin{array}{l}\text { (Program } \\
\text { Attendance) }\end{array}$ & $\begin{array}{c}\text { Encouragement- } \\
\text { Family }\end{array}$ & .933 & 1,60 & .015 & -.001 & -.453 & .469 & -.124 & -.966 & .338 \\
\hline Model 2* & Constant & & & & & 85.644 & 6.000 & & 14.273 & .000 \\
\hline $\begin{array}{l}\text { (Program } \\
\text { Attendance) }\end{array}$ & $\begin{array}{c}\text { Encouragement- } \\
\text { Friends }\end{array}$ & 7.663 & 1,58 & .117 & .101 & -1.698 & .613 & -.342 & -2.768 & $.008 * *$ \\
\hline Model 3* & Constant & & & & & 74.274 & 6.174 & & 12.030 & .000 \\
\hline $\begin{array}{l}\text { (Program } \\
\text { Attendance) }\end{array}$ & $\begin{array}{l}\text { Discouragement- } \\
\text { Family }\end{array}$ & .395 & 1,60 & .007 & -.010 & -.336 & .534 & -.081 & -.628 & .632 \\
\hline Model 4* & Constant & & & & & 78.620 & 5.795 & & 13.567 & .000 \\
\hline $\begin{array}{l}\text { (Program } \\
\text { Attendance) }\end{array}$ & $\begin{array}{l}\text { Discouragement- } \\
\text { Friends }\end{array}$ & 2.233 & 1,59 & .036 & .020 & -.881 & .590 & -.191 & -1.494 & .140 \\
\hline Model 5* & Constant & & & & & 4.773 & 5.376 & & .888 & .381 \\
\hline $\begin{array}{l}\text { (LDL } \\
\text { Cholesterol) }\end{array}$ & $\begin{array}{c}\text { Encouragement- } \\
\text { Family }\end{array}$ & 1.543 & 1,35 & .042 & .015 & -.520 & .419 & -.206 & -1.242 & .222 \\
\hline
\end{tabular}




\begin{tabular}{|c|c|c|c|c|c|c|c|c|c|c|}
\hline \multirow{2}{*}{$\begin{array}{l}\text { Model 6* } \\
\text { (LDL } \\
\text { Cholesterol) } \\
\end{array}$} & Constant & & & & & 5.211 & 5.429 & & .960 & .344 \\
\hline & $\begin{array}{c}\text { Encouragement- } \\
\text { Friends }\end{array}$ & 1.134 & 1,33 & .033 & .004 & -.553 & .520 & -.182 & -1.065 & .295 \\
\hline \multirow{2}{*}{$\begin{array}{l}\text { Model 7* } \\
\text { (LDL } \\
\text { Cholesterol) }\end{array}$} & Constant & & & & & 5.010 & 5.539 & & .905 & .372 \\
\hline & $\begin{array}{c}\text { Discouragement- } \\
\text { Family }\end{array}$ & 1.548 & 1,35 & .042 & .015 & -.565 & .454 & -.206 & -1.244 & .222 \\
\hline \multirow{2}{*}{$\begin{array}{l}\text { Model 8* } \\
\text { (LDL } \\
\text { Cholesterol) }\end{array}$} & Constant & & & & & .900 & 5.860 & & .154 & .879 \\
\hline & $\begin{array}{c}\text { Discouragement- } \\
\text { Friends } \\
\end{array}$ & .070 & 1,34 & .002 & -.027 & -.163 & .616 & -.045 & -.264 & .793 \\
\hline \multirow{2}{*}{$\begin{array}{l}\text { Model 9* } \\
\text { (A1c) }\end{array}$} & Constant & & & & & -.244 & .156 & & -1.563 & .126 \\
\hline & $\begin{array}{c}\text { Encouragement- } \\
\text { Family } \\
\end{array}$ & 2.131 & 1,41 & .049 & .026 & .018 & .012 & .222 & 1.460 & .152 \\
\hline \multirow{2}{*}{$\begin{array}{l}\text { Model 10* } \\
\text { (A1c) }\end{array}$} & Constant & & & & & -.085 & .143 & & -.596 & .555 \\
\hline & $\begin{array}{c}\text { Encouragement- } \\
\text { Friends } \\
\end{array}$ & .523 & 1,39 & .013 & -.012 & .010 & .014 & .115 & .723 & .474 \\
\hline \multirow{2}{*}{$\begin{array}{l}\text { Model 11* } \\
\text { (A1c) }\end{array}$} & Constant & & & & & -.006 & .165 & & -.038 & .970 \\
\hline & $\begin{array}{c}\text { Discouragement- } \\
\text { Family } \\
\end{array}$ & .058 & 1,41 & .001 & -.023 & .003 & .014 & -.038 & -.241 & .810 \\
\hline \multirow{2}{*}{$\begin{array}{l}\text { Model 12* } \\
\text { (A1c) }\end{array}$} & Constant & & & & & -.135 & .167 & & -.810 & .423 \\
\hline & $\begin{array}{l}\text { Discouragement- } \\
\text { Friends }\end{array}$ & .466 & 1,40 & .012 & -.013 & .011 & .017 & .107 & .683 & .499 \\
\hline
\end{tabular}

\begin{tabular}{|c|c|c|c|c|c|c|c|c|c|c|}
\hline \multicolumn{11}{|c|}{ Social Support Exercise Models } \\
\hline & & $\mathbf{F}$ & df & $\mathbf{R}^{2}$ & Adj. $\mathbf{R}^{2}$ & B & SE B & $B$ & $t$ & p-value \\
\hline \multirow{3}{*}{$\begin{array}{l}\text { Model 13* } \\
\text { (Attendance) }\end{array}$} & Constant & & & & & 75.556 & 5.459 & & 13.840 & .000 \\
\hline & Family & .995 & 1,60 & .016 & .000 & -.282 & .283 & -.128 & -.998 & .322 \\
\hline & Participation & & & & & & & & & \\
\hline \multirow{2}{*}{$\begin{array}{l}\text { Model 14* } \\
\text { (Attendance) }\end{array}$} & Constant & & & & & 75.923 & 5.963 & & 12.733 & .000 \\
\hline & Friend Participation & .989 & 1,56 & .017 & .000 & -.348 & .350 & -.132 & -.994 & .324 \\
\hline \multirow{3}{*}{$\begin{array}{l}\text { Model } 15 \\
\text { (HDL } \\
\text { Cholesterol) }\end{array}$} & Constant & & & & & 5.939 & 3.534 & & 1.681 & .100 \\
\hline & Family & 2.607 & 1,41 & .060 & .037 & -.283 & .175 & -.245 & -1.615 & .114 \\
\hline & Participation & & & & & & & & & \\
\hline \multirow{2}{*}{$\begin{array}{l}\text { Model } 16 \\
\text { (HDL } \\
\text { Cholesterol) }\end{array}$} & Constant & & & & & -2.562 & 4.298 & & -.596 & .555 \\
\hline & Friend Participation & .978 & 1,38 & .025 & -.001 & .259 & .262 & .158 & .989 & .329 \\
\hline
\end{tabular}




\begin{tabular}{lcccccccccc}
\hline Model 17 & Constant & & & & & -.089 & .149 & & -.594 & .556 \\
\cline { 2 - 9 } & $\begin{array}{c}\text { Family } \\
\text { Participation }\end{array}$ & .130 & 1,41 & .003 & -.021 & .003 & .007 & .056 & .361 & .720 \\
\hline $\begin{array}{l}\text { Model 18 } \\
\text { (A1c) }\end{array}$ & Constant & & & & & -.068 & .178 & & -.379 & .707 \\
\cline { 2 - 10 } & Friend Participation & .048 & 1,38 & .001 & -.025 & .002 & .011 & .036 & .220 & .827 \\
\hline
\end{tabular}

*Covariates for models= age, education, diabetes status, income, marital status, household size and gender

$* *$ Significant, $\mathrm{p}<0.05$

\subsection{Discussion}

Participants rated their social support positively with exercise compared to their diet. The majority of the participants indicated some level of encouragement for their diet from family and friends. For exercise, there was a much stronger level of support from family and friends than diet. While some participants seemed to have a network of family and friends to encourage them to engage in healthy eating habits, more reported they had better social support to improve their engagement in exercise.

The study results support previous lifestyle interventions that have measured social support and program attendance and have important public health findings for WV. It provides evidence that social support from family and friends is high among adult population with diabetes and pre-diabetes in the two communities used for the DPM program. Since diet and physical activity are two important components of lifestyle modification that are associated with health outcomes, social support and social problem solving should be incorporated to further improve behavior modifications in the current health interventions such as the DPM and other diabetes programs. The positive impact of social support with eating and exercise habits could result in improved program outcomes such as program attendance. Participants were older adults with approximately two-thirds currently married and living in a household of one or more individuals. Therefore, lifestyle programs could identify participants' level of social support for changing dietary and physical activity habits and tailor the needs during program implementation.

Mean social support for diet and physical activity showed participants perceived positive social support for diet and exercise habits as the overall support for each item was "very often" to "often" positive responses. Social support from family and friends for improving dietary habits included encouragement to avoid unhealthy foods, suggestion for changes to eating habits and compliments when that occurred, and support to avoid high fat/salt foods by refusing to eat and/or creating an environment of support (e.g., not bringing those food to home or eating in front of the participant). Exercise social support assessed support from family and friends for reminders to exercise, schedule changes, planned activities, ideas, and discussions. While a higher social support was noted for physical activity as compared to dietary habits, lack of perceived social support for dietary modifications, received as encouragement from friends, significantly improved program attendance. This indicates that lower perceived social support for lack of healthy dietary habits encouraged program attendance. Perhaps the complex relationship between food and health requires skills and knowledge for individuals with diabetes and prediabetes to understand its relationship, reading food labels, carbohydrate counting, and food choices and meal plans that were provided by the DPM program sessions and were of interest to 
participants who perceived less support for these by family and friends. Research shows that if a person perceives higher level of social support, it would in turn result in higher program success and a better attrition rate (Teixeira et al., 2004; Fowler, Follick \& Rickard-Figueroa, 1985; Mitchell \& Stuart, 1984; Yass, Barry \& Dacey; 1993). Hence, it makes sense that poor perceived support from family and friends for dietary habits resulted in participants to rely on their HCs and peer social support group at the DPM program sessions. Furthermore, several DPM program sessions focused on cooking demonstrations, healthy snacks/lunch/dinner discussion and activities, reading food labels, and potlucks of commonly cooked recipes (with healthy modifications) for a delicious variety that provided participants with food labels and recipes for use at home. The results also support the verbal comments made by the program participants to me (as the DPM Program Coordinator) that they had limited knowledge of meal planning and a social support network was one of the reasons that they joined the program. The participants also emphasized not only the social support received from program staff and HCs but also the food logs they completed that improved their understanding of nutritional information as well as accountability for their actions (or lack thereof) and discussions with their peers and HCs.

Unlike prior literature, neither education nor income of the participant was associated with program attendance in the multivariate model, and did not predict the outcome variables (attendance, HDL, LDL, and A1c). Perhaps the low sample size and homogeneity of the group, which consisted of mostly college-educated and middle-income individuals (as compared to the rest of the WV population) provided limited variance for these two variables. Likewise, all of the bivariate relationships with the demographic characteristics were accounted for but when placed into the regression model, they were not significant. While lack of social support in the elderly population can lead to their poor health, the unique aspect of Appalachian culture might be different as individuals live and work in enclaves of friends and family and "familism" indicates family ties that overlap economic and social ties. This might also account for the general levels of perceived social support among the participants.

Our study findings concur with Vangelisti as social support and behavioral changes resulted in both positive and negative results, simultaneously (2009). Positive outcomes of social support from friends and family was noted for encouragement to improve physical activity but lack of social support for dietary changes were noted by participants. As with other studies, an individual's support network can be made up of family members, colleagues, coworkers, friends and communities (Verheijden et al., 2005; Sarason \& Sarason, 2009). Furthermore, new sources of support, such as peers' groups in program interventions, can be helpful with behavior change. Therefore, based on the participants' reporting a low level of social support with eating habits could have been helped by including fellow participants in their social network. This unique finding has implications for future diabetes program and programmatic changes can lead to positive outcomes for dietary changes which in turn can decrease the likelihood of uncovering negativity among behavioral change (Brashers et al., 2004).

Although social support was associated independently with the DVs (A1c, HDL, and LDL), they failed to gain significance in the multivariate analysis. However, A1c, HDL and LDL were improved for participants which is very important for intervention research. Therefore, despite lack of association in model, participants resulted in better health outcomes (A1c, HDL, and LDL) from participating in the program. Irrespective of the relationship of social support with dietary and PA changes, monitoring them for process and outcome evaluations are important as well. There could be a couple of reasons why perceived social support did not 
impact diet and exercise habits and clinical outcomes: (a) low number of participants with complete data, and (b) measurement of social support was done using a single time point. In addition, the Appalachian culture might be different than other population studied in the past which have shown social support was related to clinical outcomes (Rees, Karter \& Young, 2010; Turner et al., 2010; Heisler, 2007). In addition, our participants included both individuals with diabetes and pre-diabetes and the social support for diet and physical activity modifications may differ based on their disease and co-morbidities in the DPM program. Hence, future studies are needed to confirm these results in larger sample and examine direct and buffering effect of social support among individuals by their disease status.

There were several strengths of this study. It is one of few studies that examined social support related to diet and exercise of a diabetes program with both prediabetes and diabetes individuals. Speculations of program success outside of metrics required by DPP and diabetes management programs has been of interest, but still little is known about how social support can play a role in program success or failure with lifestyle changes/improvements. Participants' levels of social support could be a crucial factor in understanding their engagement in healthy, lifestyle behaviors. Based on recruitment and eligibility of the program, the study sample was relatively generalizable in gender, marital status, and diabetes status. Additionally, diabetes status was based originally on a self-report measure, but it was confirmed with an A1c value. With the inclusion of both individuals at risk for diabetes and confirmed disease statuses (prediabetes and diabetes), multiple study sites and a wide range of participants by age groups, the study findings can be generalized to WV and other populations. However, results should be considered with the following limitations. Study variables such as demographic characteristics and survey questions (social support) were self-reported, which could have led to measurement error and misclassification bias. However, standardized questionnaires with valid and reliable scales were used, which was confirmed with this study sample based on the measure of Cronbach's a. In addition, small sample size and issues with missingness in the data were found to have a significant impact on the results. For example, even though the total sample had 70 participants, the final model only included 59 participants. For the clinical outcomes, approximately $\sim 40$ participants had baseline and post-program data, which could have limited the significance levels in the model and impacted study results.

\subsection{Conclusion and Implications}

This is one of first studies to examine social support among participants of a communitybased diabetes program that included both individuals with prediabetes and diabetes through quantitative assessment. Behavioral, program, and clinical data was used to assess the relationships among perceived social support, program session attendance and clinical outcomes (A1c, LDL and HDL). Perceived social support from family and friends for dietary changes and improved physical activity had a mixed effect on program attendance and no impact on clinical outcomes. This suggests that deprived patients benefit less from family and friends and rely more on program for support to gain skills and knowledge while they are trying to make various lifestyle changes. Given the alarmingly high rates of diabetes and prediabetes in WV, programs that provide social support for improved dietary changes and physical activity can allow for program personnel to make lifestyle programs more relevant and tailored to participant needs. Since diabetes is known to be associated with microvascular and macro vascular complications and associated health care costs, there is indeed an urgent need to further improve current diabetes intervention programs in WV. Hence, programs could be tailored to improve levels of 
social support to participants, perhaps with peers who are enrolled in the intervention or by HCs and other trained personnel who can provide the guidance and encouragement for behavioral modifications.

Lifestyle modification such as physical activity and healthy diet are recommended for diabetes self-management and prevention, nonetheless it is complex, and patients face challenges for sustained changes. Hence, it should be delivered by a multidisciplinary approach and managed by a team of health professionals as used by the DPM program. Our team consisted of professionals in public health, medicine, nutrition, exercise physiology, and nursing. Social support can be a modifiable factor in such programs and by, identifying the level of social support of participants at baseline, program implementers can understand and tailor program outcomes that are depended on lifestyle modifications. Support for participants can be also be achieved in more ways than through their current family and friends. For example, participants could improve their social support network by simply connecting with resources provided in the program sessions. Current gap between knowledge of social support and lifestyle modifications requires that level of social support of the participants as related to eating and exercise habits are examined for chronic diseases, specifically diabetes intervention research. Future research could focus on including social support measures along with the required program metrics when replicating with a new population. This expansion of research could lead to better understanding of participants in lifestyle interventions and how level of social support impacts their ability to modify lifestyle behaviors. 
CHAPTER 4

Using a qualitative technique to examine participant perceptions on social support, benefits and barriers of a West Virginia diabetes program 


\subsection{Significance}

The disproportionate burden of diabetes and prediabetes in WV makes studying the factors that influence effectiveness and outcomes of diabetes interventions a felt need in this medically underserved state. However, changing behavior is a complex process that requires an evaluation of factors that influence patient empowerment in diabetes prevention, care and education, which has not received enough consideration in community-based lifestyle programs (Van Dam et al., 2005). The literature has dedicated even less attention to the role played by familial and other forms of social support in diabetes care, and individual's assessment of benefits and barriers in lifestyle interventions. To address this gap and improve our knowledge of these factors in rural communities, effective translation of interventions is necessary for improved health outcomes (Straus et al., 2013).

\subsubsection{Why Do Qualitative Research?}

In contrast to traditional, quantitative research studies, qualitative studies are conducted to examine and analyze individual experiences, preferences, and needs (Tripp-Reimer \& Doebbeling, 2004). Identifying barriers and promoters of lifestyle interventions is a critical step in achieving positive behavior changes and health outcomes. Exploring patients' perceptions about what factors may explain poor health status among rural Appalachian patients is best done through qualitative studies like focus groups and in-depth interviews. While qualitative research tends to be exploratory and is not designed to formally test scientific hypotheses (Goh et al., 2008), it provides information that can be used to tailor the design and curricula for interventions. Likewise, the use of qualitative research techniques allows for a better understanding of community concerns for sustainability and tailoring it to community cultures and contexts (Goh et al. 2008).

Qualitative techniques and research, such as participatory research (PR), recognizes the value of including and engaging the community in research (Cargo \& Mercer, 2008; Kemmis \& McTaggart, 2000). PR is a collaborative approach that integrates academic expertise with nonacademic participants' knowledge and experiences. It allows for more comprehensive and coordinated responses to addressing public health issues. Although most researchers find value in community stakeholder input and feedback when designing interventions, few achieve full level of collaboration or comprehension gained through PR (Cargo \& Mercer, 2008).

\subsubsection{Qualitative Studies}

\subsubsection{Lifestyle Programming}

Previous qualitative studies have evaluated the link between poor health and disease (Dankwa-Mullan et al., 2010; Hayter et al., 2015; Arpey, Gaglioti \& Rosenbaum, 2017; Simon et al., 2005). The association between poor health and disease is due to the impact of social, political, and economic factors on a community or population of interest. Additionally, qualitative research has also been used to further examine factors related to behavior change for community-based programming. Factors can be related to, but are not limited to, the benefits and barriers and the participants' social support (Lambert et al., 2005; Kozica, 2013 Chan, Lok, Sea, \& Woo, 2009; Biedenweg et al., 2014). These factors are important when reviewing clinical outcomes and evaluating lifestyle programs. For example, Lambert et al. recruited overweight or obese mothers from two rural WIC clinics to explore benefits and barriers to program 
participation (2005). The authors found that physical activity, health status and fewer societal prejudices were noted as benefits among program participants, while they described low selfesteem, lack of personal effort, lack of social support, and inadequate finances as significant barriers.

Moreover, one of the greatest barriers to healthy lifestyle engagement is lack of awareness of available resources and programming (Kozica, 2013). Most individuals will engage in a behavior change if there is belief that the change is valuable and achievable (Chan, Lok, Sea, \& Woo, 2009). Individuals who participate in weight loss programs tend to share similar experiences during weight loss maintenance regardless of whether or not they maintain their weight loss (Reyes et al., 2012). These experiences can be triggered by a key motivator/benefit such as commitment to others and being part of a social community (Lidegaard, Schwennesen, Willaing, \& Faerch, 2016). Thus, understanding the contextual opinions of the local community and program participants can help ensure acceptability and feasibility of lifestyle programs (Smith, Straker, McManus, \& Fenner, 2014).

\subsubsection{Diabetes Programs}

As mentioned in Chapter 1, diabetes can be managed through healthy behavior changes. Briggs revealed an attitudinal difference exists among those who have good versus poor diabetes control. For example, individuals with good diabetes control tend to have positive transfer of energy towards other lifestyle behaviors/experiences. In addition, programs tend to appeal to participants who are more socially inclined and seeking accountability (Biedenweg et al., 2014). Moreover, Niedermann et al. concluded that identifying benefits and barriers to joint protection for arthritis (self-management technique for reducing pain) through qualitative approaches can have a positive impact on communication when individuals are burdened by diabetes (2010). Based on the previous studies mentioned, there is strong support for identifying motivators and barriers to program participation in order to improve public health programming (Lambert et al., 2005; Kozica, 2013 Chan et al., 2009; Biedenweg et al., 2014.

Moreover, an analysis of focus groups with dietary group counseling participants indicated more concrete content for their counseling discussions such as work-related factors, social support, and time management skills (Korkiakangas et al, 2011). Similarly, patients who attended clinical consultations through a diabetes center reported social support as a key factor in managing their diabetes (Black, Maitland, Hilbers \& Orinuela, 2017). More specifically, these patients mentioned involving their family and/or other social networks could improve prevention or management programming.

\subsubsection{Gap in Literature}

Qualitative studies are particularly useful when researchers need to explore participants' attitudes, feelings and behavior change in-depth. They allow for individuals to expand on their personal views and can lead to discussion of individual experiences and preference. The openness among participants can be helpful in retrieving useful information that cannot be gleaned quantitatively. Hence, we expected that qualitative data would help explain the quantitative data related to benefits, barriers and social support among DPM participants. 
While qualitative methods have been used to study lifestyle programming previously, little qualitative research exists related to diabetes prevention and management programs. Likewise, benefits and barriers and social support of lifestyle modifications have been studied previously in different populations and programs, but not together, to explore the underlying reasons for program effectiveness. As mentioned previously, qualitative approaches and analyses allow for the examination of in-depth participant information and further evaluation of program effectiveness. Therefore, the aim of this study was to explore the DPM participants' perceptions of the benefits, barriers and social support in relation to program outcomes and participation, using focus groups.

\subsubsection{Author's Lens}

In qualitative research, authors are required to provide a personal view/lens and detailed background of the subject. This ensures trustworthiness of the data and further acknowledges inherent biases that may play a role in data collection, analysis, and reporting during qualitative research. The topic of diabetes and lifestyle programming really hits home with this author. The author grew up experiencing difficulties with proper dietary habits and physical activity along with mental health issues in the home. These issues often lead to prolonged risk factors and negative health behaviors. After receiving a college education (BS and $\mathrm{MPH}$ ), the author was able to understand and learn ways to improve her own health status as well as share her knowledge with others. This was done by directly studying public health issues and completing advanced training in chronic diseases, specifically for diabetes prevention and management. Furthermore, the author took the role of Program Coordinator of the DPM program. Through this role, the author developed a close relationship with other researchers in the field and continued to learn how improve her overall health/well-being while sharing information with participants. As Program Coordinator, the author had direct interactions with the participants for most sessions, provided educational sessions, completed data collection assessments and supervised the administrative processes. Thus, the author's view and thoughts are very close to the project through close involvement in the implementation of the DPM program. However, it provides an opportunity for her to give context to most situations mentioned.

\subsection{Methods}

A secondary qualitative data analysis was conducted to explore participants' experience and satisfaction in the DPM program. Original data was collected to prove the feasibility and effectiveness of the program, specifically what participants liked or did not like in the program and suggestions for change if the program will be implemented in the subsequent years (see Chapter 1). The original qualitative data included two focus groups with $\mathrm{HCs}$, one focus group with the $\mathrm{CAB}$ in Morgantown, and three focus groups with participants from two sites in two years (1 Morgantown site in Year 1 and Morgantown and Charleston sites in Year 2). Approximately, 5-10 individuals were recruited per focus group with a total of six focus groups completed with the $\mathrm{CAB}, \mathrm{HCs}$ and participants.

For this aim, we were specifically interested in participants' experiences with benefits, barriers and social support of participating in the DPM program. Thus, data was only analyzed for the DPM participant focus groups and not the CAB or HC focus groups. 


\subsubsection{Larger Study Focus Group Development}

The RE-AIM framework was used to develop focus group scripts to evaluate the DPM participants' perceptions of the diabetes program's potential for translation to West Virginia communities (Glassgow et al., 1999) (Appendix L). These questions were developed to help capture in-depth information on participant and contextual factors that facilitate or hinder successful implementation. All guides included a set of identical core questions as well as questions specific to the focus group audience. The moderator utilized the guides and applied qualitative research expertise to probe for additional information as deemed necessary. The goal of the focus group guide was to gain insight and information to perceived benefits and barriers of the program.

A letter of request was distributed to all participants prior to the focus groups (Appendix $\mathrm{K})$. The letter provided an overview of the focus group format and process. Focus group participants were notified that answering questions was not required and that responses were kept confidential. It was also disclosed that the discussion would be audio-recorded and, therefore, anonymity could not be guaranteed. All participants signed the consent forms prior to the start of the focus groups. Two consultants, external to the DPM program personnel, skilled in qualitative research, conducted all focus groups. Note takers and a faculty member assisted the external consultants during the qualitative research data collection; DPM program PI or Project Coordinator was not involved for objectivity.

\subsubsection{Recruitment and Logistics}

As mentioned in Chapter 1, focus groups were scheduled largely based on convenience to potential participants. For this retrospective interpretation, only the following focus groups were used: 1) Year 1 Morgantown Participants, 2) Year 2 Morgantown Participants, and 3) Year 2 Charleston Participants. The focus groups took place for 60-90 minutes in private rooms in the following locations: Woodland United Methodist Church (Morgantown DPM program site) and Village Chapel Church (Charleston DPM program site).

The focus groups were planned immediately following a regularly scheduled DPM program session. Preliminary recruitment was conducted 4-6 weeks prior to the focus groups, during the DPM program sessions. Participants were given a description of the focus group process, overall purpose, and expected outcomes. Interested participants were asked to sign-up for the focus group. Follow-up phone calls were made to verify participation prior to the focus group and potential participants were given a description of the focus group process for the second time as a reminder and were also notified of the $\$ 20$ gift card incentive.

\subsubsection{Qualitative Analyses}

Transcription was contracted and completed by an approved transcription service, 'Rev', prior to data coding. Focus group audio recordings were transcribed verbatim. Notes from focus groups and demographic information of participants were examined. Directed content analysis, a secondary data analysis, was used to qualitatively explore participant social support and the benefits and barriers of the DPM program (Thorne, 1994). This type of analysis utilizes existing data to further develop themes from ideas that were captured, but not fully explored during data collection and analysis of the original study. While directed content analysis permits the 
researcher to make decisions a priori about what content will be analyzed and which codes will be used, it does allow for final codes, categories, and themes to emerge during data analysis.

All transcripts were cleaned after receiving the final versions from Rev. After that, all transcripts were independently reviewed and read by two researchers/coders i.e., the author and a qualitative research expert (hereafter identified as SS and DD). After a thorough read, a debriefing discussion session took place which included SS and DD going through each section of transcripts to ensure consistency in preliminary coding processes. After that, preliminary codes and definitions specifically related to benefits, barriers and social support regarding individual health/behaviors and DPM participation were finalized and agreed upon by both coders. After this agreement, SS and DD reviewed the transcripts again and re-coded the data to fit the agreed upon codes. Then, the two coders met to agree on how to merge or split codes into categories, which were then organized into a hierarchical structure, resulting in emergent themes (see Appendix M, N and O). In two additional meetings between SS and DD, codes were broken down into categories and sub-categories for each theme. This process of detailed reading, coding, categorizing, and debriefing with a second coder was used to improve reliability, as many qualitative researchers question the use of an intercoder agreement (e.g., kappa coefficients). Therefore, researchers have argued that qualitative research is an interpretive enterprise and should be considered appropriately (Sandelowski \& Barroso, 2007; Harry, Sturges \& Klingner, 2005; Brinkmann \& Kvale, 2015).

\subsubsection{Participant Demographics}

As mentioned previously in Chapter 1, eligibility criteria for the larger program study included those who were at risk for developing diabetes (prediabetes) or were diagnosed with diabetes, were 18 years of age or older, had a BMI of equal or greater than $24 \mathrm{~kg} / \mathrm{m} 2$ and had to be motivated to meet the Surgeon General's recommended physical activity level of at least 150 minutes per week. For three participant groups/two program sites, participants were representative of the larger program sample with $41 \%$ with prediabetes and $59 \%$ with diabetes. Participants were adults over 18 years of age and English-speaking. A total of 22 participants participated in the three focus groups, but three participants participated in both year 1 and 2 for the Morgantown site. Specifically, participant sample sizes were the following: 6 participants in focus group 1 (Year 1 Morgantown Participants), 10 participants in focus group 2 (Year 2 Morgantown Participants) and 9 participants in focus group 3 (Year 2 Charleston Participants). The mean age of the participants was 62.3 years and the majority $(91 \%)$ were Non-Hispanic White and female (77\%). Most of the participants were employed full-time (64\%) or were retired from a full-time job (32\%). Half or $50 \%$ had an income of $<=\$ 50,000$. Similar to the entire DPM program sample, the majority of the focus group participants were highly educated (73\%) having a college degree or higher, compared to the state average. A little over half or 59\% of the participants were married and $40.9 \%$ indicated they were not currently married (i.e., single, divorced or separated). 
Table 4.1

\begin{tabular}{|c|c|c|c|}
\hline Variable & Categories & $\mathbf{n}$ & $\begin{array}{l}\text { Percent (\%)/ } \\
\text { Mean +/- SD }\end{array}$ \\
\hline \multirow[t]{2}{*}{ Race } & White & 20 & 90.9 \\
\hline & $\begin{array}{c}\text { Black/African } \\
\text { American } \\
\end{array}$ & 2 & 9.1 \\
\hline \multirow[t]{2}{*}{ Gender } & Female & 17 & 77.3 \\
\hline & Male & 5 & 22.7 \\
\hline \multirow[t]{2}{*}{ Marital Status } & $\begin{array}{c}\text { Not Currently } \\
\text { Married }\end{array}$ & 9 & 40.9 \\
\hline & Currently Married & 13 & 59.1 \\
\hline \multirow[t]{2}{*}{ Education } & $\begin{array}{c}\text { High School or } \\
\text { GED }\end{array}$ & 6 & 27.3 \\
\hline & $\begin{array}{c}\text { College Degree or } \\
\text { Higher }\end{array}$ & 16 & 72.7 \\
\hline \multirow[t]{3}{*}{ Employment } & Retired & 7 & 31.8 \\
\hline & $\begin{array}{c}\text { Disabled or unable } \\
\text { to work }\end{array}$ & 1 & 4.5 \\
\hline & Full-time & 14 & 63.6 \\
\hline \multirow[t]{2}{*}{ Income } & $<=\$ 50,000$ & 15 & 68.2 \\
\hline & $>=\$ 50,000$ & 6 & 27.3 \\
\hline \multirow[t]{2}{*}{ Diabetes Status } & Prediabetes & 9 & 40.9 \\
\hline & Diabetes & 13 & 59.1 \\
\hline Age & Years & 22 & $62.32+/-10.24$ \\
\hline
\end{tabular}

\subsection{Results}

We undertook this qualitative analysis with a specific interest in discovering participants': 1) barriers to performing health behaviors and barriers to DPM program participation; 2) benefits of performing health behaviors and benefits of DPM program participation; and 3) social support related to performing health behaviors and related to DPM program participation. Thus, the results of our directed content analysis are presented under the following key thematic areas: barriers, benefits, and social support. Under each of these key thematic areas, the data were further broken down into categories related to 1) impacts on health and 2) impacts on program participation for both barriers and benefits. For the key thematic area of social support, categories of social support related to family, friends/peers, program staff and support from within emerged. Despite our specific focus on exploring benefits, barriers, and social support, it should be noted that focus group participants generally held a positive view of 
the program related to its impact on their individual health and their participation. The qualitative structure and codebook are displayed in Appendix M, N, and O.

\subsubsection{Barriers}

Barriers were defined as any negative item, activity or action that could hinder engagement/participation in healthy behavior(s) and/or the diabetes program. Question prompts about factors that hindered participant success and recommendations for program improvement elicited rich discussions about barriers faced by participants. Two distinct categories of barriers emerged: individual level health issues/behaviors and program participation. The category of individual level health issues/behaviors was related to behaviors or issues that may hinder positive health outcomes. The category of program participation was related to anything that could negatively impact participation in the diabetes program.

\subsubsection{Individual Level Health Issues and Behaviors}

Participants discussed unhealthy behaviors that they engaged in over the course of their lives that they felt led to negative health outcomes or may hinder them meeting positive health goals. Additionally, unhealthy behaviors were identified as barriers if they made engagement or participation in the program difficult, resulting in hindrances to program success. Participants described disease complications and negative impacts on their physical health/well-being. For some participants, extreme fatigue and discomfort made it challenging to meet their health goals. One participant mentioned "I mean, because your eyes get blurry and you feel terrible, you want to go to sleep all the time. It's very uncomfortable. And um, I want to stop doing that." Another stated, "And I think for about two years, I just vegetated because I didn't feel well enough to do anything."

Some participants noted that failing to recognize diabetes as a serious and complex disease, oftentimes until it was too late, was a barrier to performing certain health behaviors. One participant stated, 'I'm sitting right down the road and you've got cancer, you got heart attacks, that's the big thing. And of course, for years high blood pressure and cholesterol. So, the diabetic was really not the big thing." This participant reflected on how, at first, a diagnosis of diabetes did not feel like a serious health threat. Some participants described how they thought of diabetes as a secondary condition to or consequence of a cluster of other conditions. More specifically, diabetes was not necessarily the focus, but other comorbid conditions were for them. To the participants, diabetes was not a devastating, overwhelming threat that cancer or heart attack was to them or their families. Others reflected on how they did not believe their healthcare provider about the seriousness of the disease until they saw the symptoms themselves, as one participant noted: "I think sometimes they'll just tell you that stuff, but unless you see it, you don't want to make those changes because they aren't pleasant."

\subsection{Eating History and Current Diet Strategies}

Participants' histories with eating behaviors were identified as a significant barrier to success in the program. Their histories of eating behaviors as a barrier included previous exposure to unhealthy eating habits as well as current diet strategies. These were described as habits that may hinder their health outcomes and/or goals. Barriers included dietary patterns and needs that were different than friends, peers and/or family members, including spouses. Some 
individuals experienced issues trying to maintain healthy eating habits at restaurants or on-thego. They also discussed difficulties with managing meal decisions on evenings and weekends. Furthermore, several participants discussed challenges to making healthy food choices when they were alone; one participant stated, "it's because I'm alone, it's very easy to cheat when you're alone."

Additionally, some participants felt dependent on specific food item(s) and expressed an inability to stop eating even with future consequences. One participant said she was "addicted to sugar." Addiction to food or using food as a replacement for other substances of addiction was also mentioned as a barrier to healthy eating. One participant said:

I used to smoke, which didn't help my weight any. It helps some people, but it didn't me. Anyways, um, I used to smoke and that was a, you know. . .doing something with your hands. Yeah, right. So now, it's just moved over to the food. So that's one big thing that I really, really need to work on.

\subsection{Exercise for Weight Loss}

Barriers to exercise and weight loss played a role in interrupting participants' individual health improvement goals. Specifically, participants discussed trouble with decreasing weight through physical activity due to chronic conditions which often led to negative attitudes towards physical activity. One participant shared, "Um, the exercise for me is just. . .when they say that I cringe because it's been so hard for me. I have arthritis, especially in one knee, it's really damaged, and I'm too chicken to have it replaced." Another participant felt physical activity was more difficult than adopting healthy eating habits: “Well, there's something worse than giving up your donuts, it's exercising." Some participants lost a significant amount of weight within six months of the DPM program and discussed their frustrations when hitting a weight loss plateau. Similar frustrations were felt when they would gain back some of the initial weight they lost. One participant stated, "I get really depressed because my weight will just be going up and I have no energy and I don't want to do anything."

\subsection{Chronic and Complex Nature of Diabetes}

Participants perceived the chronic and complex nature of diabetes to be a significant barrier to performing individual-level health behaviors. Participants mentioned the impact of comorbid health conditions and previous exposure to unhealthy habits that may put them at risk for complications from diabetes in the future. Participants were cognizant of the fact that even with significant lifestyle changes, diabetes remains a significant and chronic issue in their lives. Thus, participants expressed fear and avoidance surrounding their diabetes status, noting that these feelings often worked as barriers to taking action to prevent or manage the disease. One participant, who had diabetes for over 30 years, expressed, "I'm still scared to death of diabetes, always will be."

In addition, individuals discussed how no matter what they do to manage their prediabetes or diabetes, there will always be larger hurdles to overcome due to the chronicity of the disease. Therefore, some participants mentioned that the disease is a part of their daily lives and not something they can ignore or push to the side. They were aware that diabetes and 
complications would get worse as time goes on, especially if they did not accept the diagnosis and take appropriate precautions. One participant, with prediabetes, stated the following:

I have cholesterol issues, you know, and I have high blood pressure issues and it's leading down that road (to diabetes). I mean, and the doctors already told me, you've got to get your weight down, we got to get your cholesterol down, you know and we're still watching your numbers.

\subsubsection{Decreased Program Participation}

Focus group participants were asked about barriers to program participation. Data in this category included any negative item, activity or action that could decrease engagement or participation in the diabetes program. Specific barriers that resulted in decreased engagement or participation in the program included: issues with program leaders and/or staff, difficulties with food and/or physical activity tracking and the organization and structure of the program itself.

\subsection{Program Staff}

Characteristics of the program staff (health coaches, community speakers, the principal investigator and program coordinator) emerged as a barrier for program participation. Participants described their experiences with their assigned health coaches and gave suggestions for improvements on the matching processes (how health coaches were assigned to each participant), consistency of communication, and the overall one-on-one relationship between health coach and participant. In the DPM program, the program staff assigned a health coach to each participant based on availability and expertise. Each participant was assigned a health coach to communicate with on a weekly basis to monitor their program success, goals/activities, and to provide a general feedback loop of their experience. The health coaches were the main communicators and motivators for the participants. During the focus groups, some participants mentioned that they would like to have been able to choose their health coach or have a choice to change health coaches at their discretion rather than at the discretion of the program staff. Specifically, participants proposed that health coaches and participants could be better matched based on personalities or common interests. For example, if health coaches could switch part of the way through or had some way of connecting the personalities/interests between the participants and health coaches.

In addition to communicating with participants during weekly check-ins, the health coaches were also responsible for leading the majority of in-person educational sessions. Participants remarked that the health coaches (who were mostly college-aged students) were dedicated, professional, and effective motivators; however, some suggested hiring health coaches closer to their own age. One participant mentioned the age disconnect: "Definitely had three times more life experience than they [health coaches] have." Another participant mentioned the importance of working with a health coach that can relate to the issues participants are experiencing: "But they're young and you know, aren't facing all the health issues that we are, and you know so go get some old students, you know?"

Participants also mentioned that they liked when individuals other than health coaches were brought in to lead a session, such as one that involved a chair yoga demonstration and the in-depth nutrition session that was led by a dietician. Participants also mentioned that there was a 
need for a more diverse group of program participants for example, the participant sample not including younger adults and they had wondered why that was the case. Additionally, they felt that the sessions could have been a little more interactive with help from themselves and the program staff.

\subsection{Tracking}

When asked about factors that may have impacted their participation in the program, the program's requirements for diet and physical activity tracking emerged as significant barriers. Diet and physical activity tracking included a commitment to regularly monitor and track food intake and exercise. It was recommended that the participants should monitor diet, physical activity and weight in keeping track booklets which included information on calories, carbohydrates, fats, physical activity steps/minutes and pounds. Participants discussed how tracking was one of the most difficult tasks for them due to it being time consuming. One participant mentioned, 'Probably the tracking has been the hardest. It's just keeping hypotheticals, once it gets away from you after a couple times you may as well just give it up." In addition to the time commitment, participants noted it was difficult to regain focus and recommit to tracking once they skipped a meal or day of tracking in the booklet. Some participants mentioned that the book containing calorie and nutritional content for various foods that they were given along with the tracking booklet was also difficult to navigate, such as one participant stated: "Because I just couldn't, it really was kind of hard to flip through that book, the book's a little disorganized." When dealing with the difficultly of tracking/recording of food intake and exercise, participants mentioned that it felt like "it was punishment" and that they were being penalized for eating healthier and exercising more frequently.

\subsection{Program Organization/Timeline}

Program meeting logistics emerged as a barrier to program participation among participants. The occurrence of sessions was mentioned due to the program schedule staggering off after three months into the program: weekly for the first 12 sessions, bi-weekly for the next 4 sessions and monthly for the last 6 sessions. Participants seemed to have a hard time adjusting and creating a habit once the program switched frequency of sessions, with one participant stating, "And, doing that every week, it was establishing more of a pattern of a lifestyle. But by switching then, to once every two weeks, then every month, I hadn't established that pattern yet." Additionally, the timing of sessions was a concern of the participants due to only one time/day offered at each site. Participants also feared the loss of accountability after the program ended, for instance one participant mentioned "And I really worry about the fact that after September, you know, there's not gonna be. . .big brother is not gonna be watching.”

\subsubsection{Benefits}

Benefits were defined as a positive item, activity or action that resulted in promoting healthy behavior(s) and/or diabetes program participation. Question prompts about factors that resulted in participant and program success resulted in profound discussions about benefits faced by participants. Two distinct categories of benefits emerged: positive health behaviors and social results from program participation and program elements. The category of positive health behaviors and social results from program was related to health behaviors and/or activities that result in positive health outcomes based on what they learned from the program. Likewise, the 
category of program elements is related to program effectiveness and what is working in the program for the participants.

\subsubsection{Positive Health Behaviors and Social Results from Program}

This category emerged and included any positive item, activity or action that could improve health status through behavior change. Social aspects such as camaraderie, support and commitment were mentioned as having impact on their success in the program. Their health behaviors and social outcomes of the program were a result of their knowledge, acceptance and control of diabetes.

\subsection{Camaraderie, Support and Commitment}

Participants described their experience with the program as a camaraderie and support system. It was mentioned that it was a collaborative and supportive atmosphere among peers where topics and individual experiences could be shared. Participants would chat before, during and after the program sessions and felt that they were holding each other accountable. They were in the program together and considered everyone a member of the team. They felt comfortable and could relate closely to their peers. One participant stated, "It's actually enjoyable thing to actually come and see people and hear their stories and what they're doing. That kind of helps you, yourself, because you'll say, golly they're doing it, you know so I can do it." In addition to sharing stories and feeling more accountable, participants mentioned that there was camaraderie and they believe that everything with the group had been wonderful. Several participants mentioned the act of making a commitment as having a positive impact on their continued participation and engagement. Participants described how they sought to complete the program regardless of the difficulties they faced, because they felt obligated or accountable. When asked what kept them engaged in the program, one participant revealed "I think that we agreed that we would do it and so there is a sense of obligation." Likewise, some participants mentioned that they had to be serious and honest with themselves in order to make the commitment and stick with the program.

\subsection{Food and Physical Activity Tracking}

Like the ongoing commitment to the program, there was also a commitment to change especially related to diet and exercise. As mentioned previously, participants were required to monitor diet, exercise and weight in tracking booklets. For some, tracking was challenging and served as a barrier to program participation, but for others, keeping track of nutritional values and physical activity minutes/steps seemed to help with improving eating and exercise habits. Specifically, writing things down seemed to make them feel more accountable for their actions, for example, one participant mentioned, "I was diligent about writing down things. I was motivated." It seemed like they felt more accountable with the program and felt that it was necessary to track, as one participant stated, "I found the best thing was just to write it down in the book, and by golly, if it wasn't in that book-then you didn't eat it." Participants felt that there was flexibility in goals based on exercise and diet tracking by actually accounting for their diet with the amount of exercise they were engaging, for example, one participant noted “Oh it's amazing. Loved it. One day 12 miles. I've been, the accountability with the eating has been, with the books, has been very good for me." They felt that it paid off when tracking and one 
participant shared the following: "That it pays to be honest in regards to recording your foods and fats and calories, although I don't like it a bit. It helps, it's a good thing."

\subsection{Health Outcomes and Healthy Behaviors}

Participants discussed their feedback on overall health from participating in the program and their experience related to improvement of health outcomes. Thoughts on bloodwork and changes in clinical results, weight loss and medication regime changes seemed to be exciting and interesting to the participants. One participant mentioned, "I've been able to go off of one of my two pills a day and off my cholesterol medicine." In addition, improvements and gains from engaging in physical activity regularly along with diet habits may have improved their health outcomes and/or goals. One participant described their doctor's reaction to their clinical results after participating in the program: "When I went back to the doctor, and I had told him, before it had been like, you know, 8.5, and I said, give me time to work on it. And I went back six months later and he took the A1C and it was 7.3. I think he about fell off the stool." Participants discussed that the lifestyle changes they employed, such as healthy eating and physical activity, often led to drastic positive changes in the health outcomes.

\subsection{Knowledge, Acceptance and Control of Diabetes}

While some participants felt frightened about the chronic nature of diabetes and listed it as a barrier to sustaining motivation for engaging in healthy behaviors, many felt that accepting the diagnosis and learning to prevent and/or manage the disease could enable them to strive for better health and well-being. One participant noted that the diagnosis helped them to take control of their life:

So, um, you know, this has, was something I think that brings a balance and an understanding and more depth to the issues and problems, than what we normally deal with on a day to day basis. And we sort of operate like a pinball in life, and allows us to control that, and realize that we don't have to be the pinball just bouncing around.

Another participant said, “I think I'm happier. Because I have, I know I have control.” In addition to control, participants discussed how the program gave them motivation to incorporate these changes long-term, and one participant stated, “I think it's manageable if you own it." It was mentioned that every person managed diabetes differently and that symptoms and treatments varied from person to person but being able to experience significant changes as a result of program participation was a huge motivator for many participants. One participant mentioned, "I Ifeel] much more hopeful and physically, I feel so much better. I can tell a huge difference already."

\subsubsection{Program Elements}

This category was defined as a positive item, activity or action that could increase engagement/participation in the diabetes program. It was further reviewed and divided into two subcategories which are health coaches/program leaders and program organization/timeline.

\subsection{Health Coaches and Program Leaders}


Participants valued their experience with the health coaches and program leaders. They seemed to like the diversity between the health coaches and division of labor for the program such as one participant revealed:

I don't know if it's because they were young and energetic, they brought a lot, enthusiastic. And it was just kinda fun to see who was gonna be here each week, because they kind of rotated around and that was neat too. And they all were very personable, very pleasant, personable.

The level of communication with the program leaders and the relationship between the participants and health coaches seemed to outweigh their difficulties with adhering to the program activities. They mentioned that having all of the information available and the knowledge of the team has been worthwhile along with feedback on the bloodwork and other program measures. They also enjoyed when the program sessions included outside speakers who presented on new topics and brought in new interactive activities for them to try out on their own. Lastly, the participants provided positive feedback and felt that they could trust their program leaders, for instance on participant shared:

And it's nice to know that young, that there are young people that do care. That take their time out, and when you do talk to them they're very informative, you know, they don't shove you away, they don't give you short answers, they're willing to share what they know and help you in any way they can.

\subsection{Organization/Timeline}

Program organization and timeline were mentioned when discussing program implementation and success. Participants felt that the physical workflow of the program worked for them, including the program curriculum, schedule of sessions, session setup and timing of the meetings. As mentioned previously, program sessions lasted for an hour and 15 minutes for 22 sessions that started weekly, went to biweekly then monthly after 6 months. Several participants mentioned that the schedule and timing "worked perfect" for them and others mentioned that they were glad the program began with weekly sessions such as one participant noted: “I'm glad we did every week from the get go, plus if it had been spaced out the other way I wouldn't hung in there at all." Moreover, the site location and room setup for the sessions was appropriate and convenient for them. Participants were grateful for having the program sessions in their local, community church. It appears that participants felt comfortable with the location and day of the week that was chosen, as one participant stated, "This has been a good location to me but looking back, any other day but Sunday would not have worked for me.” Therefore, it gave the impression their overall success in the program was in result of their experience and outcomes from the program sessions.

\subsubsection{Social Support}

Social support was defined as aid/assistance occurring within the context of a social network. This could be influences and interactions with family, friends, peers and others in the community. When discussing social support, the following subcategories emerged from the focus group data: social support received from family, friends/coworkers, program staff/fellow participants, and support from within. 


\subsubsection{Family}

This type of support was received from family members on program participation and healthy lifestyles. Additionally, their family history of diabetes, the risk factors involved, and complications were discussed as motivating support, for example one participant stated, "Yes, it's, it's really scary. We have um, what we call a first cousin's reunion and it's like, that's one of the things we ask each other. How's your diabetes, you know. We talk about it all the time because everyone has it." It appears that diabetes and its complications is in regular conversation between family members and it is known across family members thus they can share experiences, for instance a participant mentioned "Um, mother-in-law had diabetes, husband's pre-diabetic, I'm pre-diabetic so, we did about the same thing. We're down a few pounds, and yay, a couple dress sizes" along with motivations to use technology to track between family members. Participants tend to set goals to meet their family member's expectations and will strive to do better based on their support from their family such as one participant stated:

I have a goal. I have a son that lives in Tokyo and another that lives in Juneau, Alaska and they're both walkers and talkers so, like, my son up in Alaska, we have to get out on the beach and we have to keep going because he doesn't like to stop and wait for me to catch a breath or anything. So I have to build of my stamina in order to be able to uh, keep, keep up with him so that I don't need a phone to talk to him.

\subsubsection{Friends/Coworkers}

Friends and coworkers provided support to participants on their program participation and changes in health-related behaviors. Some participants' actions were impacted by their friendships. For example, one participant described, "So you have to have something else, you know. And if I go out with friends my best friends, the women that I know, we're all widows. They know that I'm not supposed to eat certain things, they'll say "Don't have, don't order that. Order something else." In addition to keeping each other accountable, participants seemed to have a bond with their friends that helped provide motivation for changes in health behaviors, for example a participant revealed 'I didn't think that that first month that our friendship was going to last. And then when she started to feel some benefits, I think she's, she's talking to me again." Participants seemed to rely on the benefits of a relationship with a friend and really took pride in their friendship. Friendships seemed to be a motivating factor for several participants such as one participant mentioned "And I think for me, I had my best friend and I wasn't going to let her down. She wasn't going to let me down after she decides she wasn't going to kill me.” In addition to accountability for healthy eating, the benefits received from fellow friends, and the commitment to keep each other going, participants also had a commitment to exercise with their friends such as one participant shared:

"That's why I belong to health works and I like that'cause I like the group exercise 'cause it's kinda like this support group, 'cause you go in, if you have sort of friends that always go there and do it, then it makes you go 'cause you have to all the time, will miss it, and then it's just you weigh in or talk about some of the things and stuff so that can, you know, help." 


\subsubsection{Health Coaches/Program Leaders/Fellow Participants}

Most participants received support and had positive relationships with the health coaches and/or program leaders. Participants felt that they were held accountable for their actions and changes that needed to be made to prevent or manage their disease, for example a participant stated, "I do well if I have to answer to somebody because it keeps me more consistent and in control. Um, so, that's initially how I sort of came down this road to, join.” Moreover, participants had received motivation from their peers, for instance one participant said "I mean it's because of the people around and because of the way that, you know, everybody has reacted and everything. And so, you know, sure, the activity and, and having people, they, everybody motivates you." Additionally, participants could relate and share experiences based on the connectedness between each other, such as a participant revealed "Just having other people who are in the same boat and have the same experiences." Participants received motivational support from their fellow participants on program participation and healthy lifestyles by creating a loyal support system among each other.

\subsubsection{Support from Within}

Some participants mentioned that they were their own motivating factor and support system. This program was to do something for themselves and to be their own motivation without relying on someone else, for example one participant mentioned, "And it's given me some place to go, so. For me it's been good." They motivated themselves on how much they would participate and improve their lifestyle based on the program. Participants mentioned that they could not always rely on family members and/or friends in the time of need and that they were responsible for their own well-being and health. At the end of the day, some participants felt as if they were in charge of their own fate such as a participant described:

You have to learn to love yourself because you're not going to take care of yourself unless you love yourself. And I wondered for a while what she meant by that but then when this come up, this is for me. This isn't for anybody else, my husband or family, no one, it's for me. Just for me and I look at it like, this is me loving myself because I'm giving myself this gift that my life is going to be better.

\subsubsection{Conclusion of Results}

In summary, participants had positive feedback and concerns related to improving their health to help prevent or manage their diabetes status. There were a few barriers that covered the chronicity of the disease, behavior change and how the program was organized. However, there were distinct benefits provided, which were related to commitment, their health outcomes, knowledge and acceptance of the disease, the program staff and program organization. Additionally, social support had a strong impact on their success in the program and their daily lives. Overall, participants seemed to be able to balance the positive and negative to control and manage their condition and complications.

\subsection{Discussion}

This content analysis of focus group data confirmed the authors' perceptions of the diabetes program in the view of a participant. These data provided great insights into what worked and did not work for DPM participants regarding making significant changes to their 
lifestyles and their overall participation in the program. While there were barriers noted, participants experienced numerous benefits of the program and there were many factors that helped keep them engaged in the program including social support. Most participants benefited from the program through the improvement of health outcomes and finally finding an acceptance and/or control of their disease status. Alternatively, some barriers seemed to be related to past history of their exercise and diet habits, and the chronicity and longevity of the disease. However, the program staff, tracking and program organization served as both benefits and barriers. Results indicated some minor modifications to the program elements in the future can result in improvement in overall program implementation.

Study findings concur with previous research on lifestyle programming. Specifically, benefits and barriers related to family history and eating habits were noted by Rovner et al. (2010). Results confirm findings from Rovner et al. that suggest dietary habits and tracking are easier if they are family-focused or if the family is in support of the lifestyle changes. Likewise, similar to our study findings, Korkiakangas et al., reported participants had a positive attitude towards increasing exercise. Unequivocally, participants shared minimal barriers related to exercise and confirmed that social support could be an asset for behavior change. As with our study, Chan et al. found that lack of social support could be a barrier but could also to be a motivating factor for healthy lifestyle improvements. In addition, our findings suggest that invited speakers (e.g., nutritionists) are a valuable source of information and are helpful for improving knowledge of program participants. Furthermore, our results are similar to those described by Johnson and Melton (2016) which explained information on the utilization of diabetes programming. Our study provided a more in-depth understanding of participant perceptions after they completed the program. Additionally, our findings take a step further with including results from those with diabetes and prediabetes, by identifying their relationships with one or more social support groups.

\subsubsection{Strengths}

Exploration of the directed content analysis for the benefits, barriers and social support, further explained the participants' views of the program, making it possible to modify and utilize the pilot DPM program in other settings and locations. Specifically, the Year 1 focus group analysis was used to make process changes and modify program techniques and implementation in Year 2, except for changing of the curriculum. This study captured information from individuals with both prediabetes and diabetes who attended the same program sessions, which is insightful. In addition, participants seemed to like the setup of the focus group interviews. They felt like they were in a regular session since all their fellow participants were in the room together. They had mentioned that they could share as they did in the program sessions.

Additionally, multiple characteristics of this study work to ensure trustworthiness and internal validity related to study findings (Lincoln \& Guba, 1985). This study met the objectives for all four criteria related to ensuring trustworthiness in qualitative research: credibility, transferability, dependability and confirmability (Shenton, 2004). Investigator triangulation between SS and DD and peer review/debriefing ensured credibility and confirmability of findings thus providing the "truth" of the findings and neutrality that was shaped by the respondents. Additionally, transferability was ensured by having a rich, detailed, thick 
description of the findings, which included information on sites, participants, and methods. By using a second coder (DD), interrater reliability or intercoder agreement was ensured by SS and DD in agreement on code names, coded passages, ways to code, and defining and deciding on the hierarchical structure. This resulted in dependability and reliability of the findings.

Furthermore, thematic saturation was reached by coders which reinforced the internal validity of study. This study confirms Creswell's recommendations for having at least two procedures in any given study to ensure trustworthiness of the data (2013).

\subsubsection{Limitations}

Despite the strengths provided, this study had several limitations. Firstly, the data was already collected for the DPM program, so the questions/responses could not be adjusted to understand the specific research questions as it was analyzed as a secondary data analysis through directed content analysis. Likewise, participants may have shared more personal experiences or details if they were in an individual interview instead of a larger focus group setting. Another limitation was not using the member-checking technique, which is used to double check that the transcripts were accurate and represent selected themes. Additionally, triangulation was lacking for this study; specifically, this method was limited due to the source being from one program and two investigators involved. In future studies, it would be good to have more triangulation in the study. However, some source triangulation was ensured by including participants from multiple locations/sites and with two disease conditions (prediabetes and diabetes). Moreover, participants were limited to the DPM program, so the transferability of the study was limited based on race, ethnicity, gender and education-level distributions. In addition, the sample had mostly college-educated, Caucasian females. These results could vary in future studies with diverse distribution of race, ethnicity, educational-level and/or gender. Hence, future studies should be replicated with a diverse population to ensure and understand how our results are applicable to other areas.

\subsection{Conclusions and Future Directions}

Study findings provided insightful information on what the benefits, barriers and levels of social support were for participants involved in a diabetes, lifestyle program. This information can be useful when developing, implementing and/or evaluating future interventions. These findings will allow for researchers or community leaders to have the tools to dig deeper and ask the right questions in the appropriate way to receive feedback from the participants. Most of the benefits, barriers and levels of social support could be applicable to other lifestyle interventions despite the chronic condition and/or population being studied. These findings may be generalizable to other programs outside diabetes lifestyle programming. Results of this study should be explored further in future research with a more diverse population.

This research is especially important due to the limited amount of research in the area. Researchers tend to assess all the required metrics such as clinical outcomes (A1c, lipid profile), basic behavioral outcomes (quality of life, health status) and anthropometrics (weight, attendance) with lifestyle programming, but do not take the additional step to understand their participants' perceptions. Based on the author's firsthand experiences, participants are willing to share additional information to take the step further such as detailed information on benefits and barriers of the program and their perceived level of social support. These findings could provide 
researchers with the missing puzzle piece to ensure program effectiveness with their participants. It allows for participants to voice their concerns related to the program and their individual health, which could be the breakthrough for lifestyle programming improvement and sustainability. 
CHAPTER 5

Conclusion 


\subsection{Summary of Findings}

Despite rising diabetes rates and what is known with diabetes complications and lifestyle modifications, there remain factors that have not been thoroughly addressed or explored for programmatic successes and effectiveness. Specifically, the underlying reasons on why or why not participants engage in lifestyle modifications and participate in programs. As mentioned previously, lifestyle programs tend to monitor the basic required metrics (attendance, weight and physical activity) but do not further evaluate the underlying factors that impact those metrics. These underlying factors could be benefits, barriers, social support or other non-programmatic measures. Factors like benefits, barriers and social support can play a role in success or failure of participants' engaging/participating in lifestyle modifications and long-term health outcomes. Thus, these factors can explain why the program is or is not successful and how it can be modified in the future.

For this research, three individual studies examined participant perceptions of benefits, barriers and social support in diabetes lifestyle program through quantitative and qualitative secondary data analyses. Study 1 (Chapter 2) identified participants' benefits and barriers through a survey developed by the author to understand the relationship between program attendance and percent body weight. Study 2 (Chapter 3) assessed participants' social support for dietary changes and improved physical activity or exercise habits with program outcomes (attendance, HDL, LDL and A1c) through standardized, validated surveys. Study 3 (Chapter 4) used a qualitative technique to examine participant perceptions on social support, benefits and barriers. With all three studies, there were several findings of importance. Specifically, the following important findings were found for the DPM participants: barriers has a significant relationship with percent body weight when controlling for program attendance, limited social support from family and friends resulted in higher attendance for program educational sessions and finally most participants benefited from the program and suggested with minimal programmatic changes for future DPM program implementation.

\subsection{Reflection on Results}

All three studies contributed to the literature, filled gaps and proposed next steps for future research. The findings from Chapter 2 and 3 set the stage for the qualitative analysis completed in Chapter 4. Chapter 2 and 3 identified the benefits and barriers of the program and the level of social support from family and friends for changes to diet and physical activity habits for participants. Chapter 4 built on these results by providing an in-depth analysis of these findings. Benefits, barriers and social support were further defined and broken down to thoroughly paint a picture of participants' perceptions on their individual health/behaviors and their program participation. For both Chapter 2 and 3, findings were similar between diabetes statuses (prediabetes and diabetes) and program sites (Morgantown and Charleston) in that the groups were not significantly different. Additionally, these two chapters ( 2 and 3 ) used a similar statistical analysis (multiple linear regression) to assess participant behaviors. Thus, all three studies had comparable results and built on each other to present an understanding of the participants' perceptions of a diabetes lifestyle program.

Furthermore, two quantitative aims (Chapter 2 and 3) with the qualitative aim (Chapter 4) were used to understand the participants' in-depth perceptions of benefits and barriers of the 
program and their individual health along with their level of social support. Results from the quantitative aims were similar to the qualitative aim when comparing their perceptions from surveys to the focus groups. Most of the same benefits were found for both aims: health coach or program leader, program materials, changing their lifestyle based on program, sharing program results and understanding their health status, disease and complications. However, the understanding of their overall satisfaction of the program was thoroughly explained in the qualitative aim. Additionally, some of the same barriers were found: health status, work, and family activities. Also, participants' level of social support was found to be consistent among aims. Therefore, triangulation of data sources and findings were a success in understanding participants' benefits, barriers and social support.

\subsection{Strengths and Limitations}

This was one of the first studies to analyze clinical, social and behavioral factors and anthropometrics data among participants with both prediabetes and diabetes who engaged in a 12-month diabetes prevention and management program. Additionally, this was one of few studies that explored the feasibility of benefits and barriers of diabetes lifestyle program that included both individuals at risk for diabetes (prediabetes) and those with diabetes. Since benefits and barriers along with social support are outside the realm of what is regularly collected in lifestyle programs to assess program effectiveness, thus, not many studies have mapped these three factors to lifestyle program outcomes.

Moreover, there were several strengths with all three individual studies. Firstly, Chapter 2 confirmed that a newly identified questionnaire could address benefits and barriers and provided to be an important predictor of success with lifestyle programs. For Chapter 3, this study helped to identify the importance of social support through standardized, validated survey questions with program metrics such as attendance. Low levels of perceived social support directly impacted program attendance and these participants engaged in the program even with a low level of social support. Additionally, Chapter 4 further explained the participant view of these important factors and ensured trustworthiness of the analysis with the use of a second coder, which provided the opportunity for having a rich description, peer debriefing and triangulation.

Several limitations were found with the three studies. Firstly, the questionnaire and focus group data were self-report and could have resulted in some biases such as self-report bias, measurement error, misclassification bias and social desirability bias. Specifically, the benefits and barriers survey was a newly, developed tool that has only been pilot tested with this study and has not been implemented with other audiences or studies. For both Chapter 2 and 3, the sample size was small and there were some issues with missingness in the data. In addition, some of the clinical measures were collected in the most simplistic way for the project and participants rather than the most studied/appropriate for analyses. The secondary analysis limited questions/responses to what was already collected in the larger program study, so they could not be adjusted to fit specific research questions. Additionally, the member-checking technique was not used with this study. However, the data was reviewed for clarity prior to completing the secondary data analysis. Overall triangulation was lacking for this study due to only having one source of data from one program. However, for this dissertation, triangulation was achieved through examining both the quantitative and qualitative data together. While the results were limited to the small group of DPM participants and the lack of diversity, the participants were representative of the WV population in ethnicity and marital status. Hence, future studies should 
be replicated with diverse samples (e.g., young adults, minorities, males, low SES) to ensure the generalizability of results.

\subsection{Significance and Future Directions}

The expected outcome was for the qualitative data to help explain the quantitative data for benefits, barriers and social support. Qualitative research can provide more of an in-depth understanding outside of the quantitative analysis of data. It provided triangulation with the multiple methods approach, which helped explain how the participants felt about the DPM program. By analyzing qualitatively and quantitatively for the benefits, barriers and social support, it further explained the participant view of the program, so that the pilot DPM program could potentially be utilized in other settings and locations.

Quantitative and qualitative approaches were used to triangulate data from program participants. Survey data along with clinical measures and focus groups were used to thoroughly conceptualize and comprehend the program effectiveness and feasibility of the DPM program. Examining these factors of the DPM program in the greater community can allow for program coordinators to culturally and appropriately tailor future diabetes programs. These results will help facilitate development of nutrition, physical activity and self-management educational materials that reflect the benefits, barriers and social support of a WV community. It allows for participants to voice their concerns related to the program and their individual health, which could be the breakthrough for lifestyle programming improvement and sustainability. 
Appendix A

\begin{tabular}{|c|c|c|}
\hline Month & Recommended Schedule & Curriculum \\
\hline \multirow[t]{4}{*}{1} & Weekly & Welcome to the Program \\
\hline & Weekly & $\begin{array}{l}\text { Be a Fat and Calorie } \\
\text { Detective }\end{array}$ \\
\hline & Weekly & Healthy Eating \\
\hline & Weekly & Move Those Muscles \\
\hline \multirow[t]{4}{*}{2} & Weekly & Tip the Calorie Balance \\
\hline & Weekly & $\begin{array}{l}\text { Take Charge of What's } \\
\text { Around You }\end{array}$ \\
\hline & Weekly & Problem Solving \\
\hline & Weekly & $\begin{array}{l}\text { Four Keys to Healthy Eating } \\
\text { Out }\end{array}$ \\
\hline \multirow[t]{4}{*}{3} & Weekly & $\begin{array}{l}\text { Slippery Slope of Lifestyle } \\
\text { Change }\end{array}$ \\
\hline & Weekly & Jump Start Your Activity Plan \\
\hline & Weekly & $\begin{array}{l}\text { Make Social Cues Work for } \\
\text { You }\end{array}$ \\
\hline & Weekly & Ways to Stay Motivated \\
\hline \multirow[t]{2}{*}{4} & Biweekly & $\begin{array}{l}\text { Prepare for Long Term Self- } \\
\text { Management }\end{array}$ \\
\hline & Biweekly & More Volume, Fewer Calories \\
\hline 5 & Monthly & Balance Your Thoughts \\
\hline 6 & Monthly & $\begin{array}{l}\text { Strengthen Your Exercise } \\
\text { Program }\end{array}$ \\
\hline 7 & Monthly & Mindful Eating \\
\hline 8 & Monthly & Stress and Time Management \\
\hline 9 & Monthly & Standing Up for Your Health \\
\hline 10 & Monthly & Heart Health \\
\hline 11 & Monthly & $\begin{array}{l}\text { Stretching: The Truth About } \\
\text { Flexibility }\end{array}$ \\
\hline 12 & Monthly & $\begin{array}{l}\text { Looking Back and Looking } \\
\text { Forward }\end{array}$ \\
\hline
\end{tabular}




\section{Appendix B \\ Benefits and Barriers of Diabetes Prevention and Management Programs}

This survey will give us input of the benefits and barriers of diabetes programs in West Virginia. This input will help assist us in making improvements or adjustments to our Diabetes Prevention and Management Program. Your input will make a difference. Thank you in advance for your participation!

PARTICIPANT ID

Please fill in the blank with the most appropriate answer (letter or number).

1. What is the first letter of your first name?

2. What is the first letter of your middle name (if none, please put $\mathrm{X}$ )?

3. In what month were you born (designate number, eg: "09" for September)?

4. What day of the month were you born (designate number, eg: "20" for February 20)?

\section{HISTORY}

\begin{tabular}{|c|c|c|}
\hline Please circle the response option & & \\
\hline $\begin{array}{c}\text { 13. Does someone in your family have diabetes? (Type 1 or Type 2: } \\
\text { Parents, siblings or children) }\end{array}$ & Yes & No \\
\hline $\begin{array}{c}\text { 14. Does someone in your family have heart disease? (Parents, } \\
\text { siblings or children) }\end{array}$ & Yes & No \\
\hline $\begin{array}{c}\text { 15. Have you ever been diagnosed with pre-diabetes by a health } \\
\text { professional (doctor, nurse, etc.)? }\end{array}$ & Yes & No \\
\hline $\begin{array}{c}\text { 16. Have you ever been diagnosed with diabetes by a health } \\
\text { professional (doctor, nurse, etc.)? }\end{array}$ & Yes & No \\
\hline
\end{tabular}

\section{PHYSICAL AND MENTAL HEALTH}

\begin{tabular}{|c|c|c|c|c|c|}
\hline \multicolumn{6}{|l|}{ Please circle the response option } \\
\hline $\begin{array}{l}\text { 17. Compared to other people } \\
\text { your age, how do you rate } \\
\text { your physical health? }\end{array}$ & Poor & Fair & Good & $\begin{array}{l}\text { Very } \\
\text { Good }\end{array}$ & Excellent \\
\hline $\begin{array}{l}\text { 18. Compared to other people } \\
\text { your age, how do you rate } \\
\text { your mental health? }\end{array}$ & Poor & Fair & Good & $\begin{array}{l}\text { Very } \\
\text { Good }\end{array}$ & Excellent \\
\hline
\end{tabular}




\section{HEALTH LITERACY}

\section{Please circle the best response option for each question}

\begin{tabular}{|l|l|l|l|l|l|}
\hline \multicolumn{5}{|c|}{ Please circle the best response option for each question } & \\
\hline $\begin{array}{l}\text { 19. If you need to go to the } \\
\text { doctor, clinic or hospital, } \\
\text { how confident are you in } \\
\text { filling out the medical } \\
\text { forms by yourself? }\end{array}$ & $\begin{array}{c}\text { Not at all } \\
\text { confident }\end{array}$ & $\begin{array}{c}\text { A little } \\
\text { confident }\end{array}$ & $\begin{array}{c}\text { Somewhat } \\
\text { confident }\end{array}$ & $\begin{array}{c}\text { Quite } \\
\text { confident }\end{array}$ & $\begin{array}{c}\text { Extremely } \\
\text { confident }\end{array}$ \\
\hline $\begin{array}{l}\text { 20. How often do you have } \\
\text { someone (family member } \\
\text { or staff at the clinic or } \\
\text { hospital) help you to read } \\
\text { health or medical forms? }\end{array}$ & Always & Often & Sometimes & Occasionally & Never \\
\hline $\begin{array}{l}\text { 21. How often do you have } \\
\text { problems learning about } \\
\text { your health because of } \\
\text { trouble understanding } \\
\text { written health information? }\end{array}$ & Always & Often & Sometimes & Occasionally & Never \\
\hline \begin{tabular}{|l|l|l|l|l|} 
22. How often do you have \\
trouble understanding what
\end{tabular} & Always & Often & Sometimes & Occasionally & Never \\
\hline $\begin{array}{l}\text { your doctor, nurse, or } \\
\text { pharmacist (druggist) tells } \\
\text { you about your health or } \\
\text { about treatments? }\end{array}$ & & & & & \\
\hline $\begin{array}{l}\text { 23. How often do you have } \\
\text { trouble remembering } \\
\text { instructions from the } \\
\text { doctor, nurse or } \\
\text { pharmacist (druggist) after } \\
\text { you get home? }\end{array}$ & Always & Often & Sometimes & Occasionally & Never \\
\hline
\end{tabular}

\section{PROGRAM INFORMATION:}

24. Have you participated in any diabetes prevention and/or management programs?
A) Yes, Please complete Section B participation!
B) No, Please STOP. Thank you for your

\section{SECTION B}

25. What diabetes prevention and/or management program did you most recently participate in? 

A) Diabetes Prevention and Management Program
B) Dining with Diabetes
C) Group Lifestyle Balance
D) Other:

\section{SATISFACTION OF PROGRAM}

In the table that follows, please select one answer for each question by circling the appropriate number. Remember, 5 is the highest, 1 is the lowest, and 0 is for notapplicable.

\begin{tabular}{|c|c|c|c|c|c|c|}
\hline & $\begin{array}{c}\text { Not } \\
\text { Applicable }\end{array}$ & $\begin{array}{c}\text { Strongly } \\
\text { Disagree }\end{array}$ & Disagree & Neutral & Agree & $\begin{array}{c}\text { Strongly } \\
\text { Agree }\end{array}$ \\
\hline $\begin{array}{c}\text { 26. I have been screened for } \\
\text { diabetes }\end{array}$ & 0 & 1 & 2 & 3 & 4 & 5 \\
\hline $\begin{array}{c}\text { 27. My health provider has } \\
\text { informed me of my risk for } \\
\text { diabetes }\end{array}$ & 0 & 1 & 2 & 3 & 4 & 5 \\
\hline $\begin{array}{c}\text { 28. Location of program was } \\
\text { convenient }\end{array}$ & 0 & 1 & 2 & 3 & 4 & 5 \\
\hline $\begin{array}{c}\text { 29. Transportation is a barrier for } \\
\text { me }\end{array}$ & 0 & 1 & 2 & 3 & 4 & 5 \\
\hline $\begin{array}{c}\text { 30. The weather kept me from } \\
\text { attending some program } \\
\text { sessions }\end{array}$ & 0 & 1 & 2 & 3 & 4 & 5 \\
\hline $\begin{array}{c}\text { 31. My health status kept me } \\
\text { from attending some program } \\
\text { sessions }\end{array}$ & 0 & 1 & 2 & 3 & 4 & 5 \\
\hline $\begin{array}{c}\text { 32. My work kept me from } \\
\text { attending some program } \\
\text { sessions }\end{array}$ & 0 & 1 & 2 & 3 & 4 & 5 \\
\hline $\begin{array}{c}\text { 33. Family activities kept me from } \\
\text { attending some program }\end{array}$ & 0 & 1 & 2 & 3 & 4 & 5 \\
\hline sessions
\end{tabular}

\begin{tabular}{|c|c|c|c|c|c|c|}
\hline $\begin{array}{l}\text { 34. Fasting before bloodwork was } \\
\text { easy }\end{array}$ & 0 & 1 & 2 & 3 & 4 & 5 \\
\hline $\begin{array}{l}\text { 35. I feel uncomfortable giving } \\
\text { blood }\end{array}$ & 0 & 1 & 2 & 3 & 4 & 5 \\
\hline $\begin{array}{l}\text { 36. The health coach or program } \\
\text { leader was encouraging and } \\
\text { helpful }\end{array}$ & 0 & 1 & 2 & 3 & 4 & 5 \\
\hline $\begin{array}{l}\text { 37. The program materials/ } \\
\text { demonstrations were } \\
\text { informative }\end{array}$ & 0 & 1 & 2 & 3 & 4 & 5 \\
\hline $\begin{array}{l}\text { 38. I changed my lifestyle as a } \\
\text { result of the program }\end{array}$ & 0 & 1 & 2 & 3 & 4 & 5 \\
\hline
\end{tabular}




\begin{tabular}{|c|c|c|c|c|c|c|}
\hline $\begin{array}{l}\text { 39. I will discuss my program } \\
\text { results with my health } \\
\text { provider }\end{array}$ & 0 & 1 & 2 & 3 & 4 & 5 \\
\hline $\begin{array}{l}\text { 40. I will discuss my results with } \\
\text { my family and/or friends }\end{array}$ & 0 & 1 & 2 & 3 & 4 & 5 \\
\hline $\begin{array}{l}\text { 41. The program helped me } \\
\text { understand the risks } \\
\text { associated with diabetes } \\
\text { and/or it's complications }\end{array}$ & 0 & 1 & 2 & 3 & 4 & 5 \\
\hline $\begin{array}{l}\text { 42. The program helped me to } \\
\text { understand how to prevent } \\
\text { and/or manage diabetes }\end{array}$ & 0 & 1 & 2 & 3 & 4 & 5 \\
\hline $\begin{array}{l}\text { 43. Overall, I was satisfied with } \\
\text { the program }\end{array}$ & 0 & 1 & 2 & 3 & 4 & 5 \\
\hline
\end{tabular}

Please provide a written explanation for the remainder of the survey questions (38-41).

44. What barriers (if any) kept you from attending the program sessions?

45. Please provide suggestions on how to improve the program in the future.

46. What benefits have you received from the program?

47. What was the most interesting thing that you learned throughout the program?

Thank you for your participation in this survey. Your responses will make a difference in our program! 


\title{
Appendix C
}

Journal of

Clinical Medicine

Article

\section{Perceived Benefits and Barriers of a Community-Based Diabetes Prevention and Management Program}

\author{
Samantha Shawley-Brzoska * and Ranjita Misra \\ School of Public Health, West Virginia University, Morgantown, WV 26506, USA; ramisra@hsc.wvu.edu \\ *Correspondence: sshawley@hsc.wvu.edu; Tel.: +1-681-285-9769
}

Received: 16 February 2018; Accepted: 9 March 2018; Published: 13 March 2018

\begin{abstract}
This study examined the perceptions of benefits of and barriers to participating in a community-based diabetes program to improve program effectiveness. The Diabetes Prevention and Management (DPM) program was a twenty-two session, 1-year program, modeled after the evidence-based National Diabetes Prevention Program and AADE7 Self-Care Behaviors framework. Community-based participatory research approach was used to culturally tailor the curriculum. Participants included overweight or obese adults with dysglycemia. A benefits and barriers survey was developed to gather information on participants' perception of the program, as well as information on demographics and health literacy levels. Eighty-nine adults participated in the DPM program (73\% females; $62 \%$ diabetic; $77 \%$ had adequate health literacy); $79 \%$ of participants completed the benefits and barriers survey. Principal component analysis indicated two components representing benefits (Cronbach's $\alpha=0.83$ ) and barriers $(\alpha=0.65)$. The majority perceived high benefits and low barriers to program participation; benefits included helpful interaction with health coach or program leader (73\%), improved lifestyle modification (65\%) due to the program, and satisfaction with the program (75\%). Open-ended questions confirmed themes related to benefits of program participation, suggestion for programmatic improvements as well as barriers to participation. Participant feedback could be used to guide interventions and tailor future program implementation.
\end{abstract}

Keywords: diabetes; lifestyle modifications; intervention; benefits; barriers; health literacy

\section{Introduction}

Diabetes affects 30.3 million Americans (9.4\% of the population) and contributes to early morbidity and mortality [1]. In addition, 84.1 million Americans or $33.9 \%$ have prediabetes [1]. Diabetes is especially prevalent in Appalachian areas [2], particularly West Virginia, the only all-Appalachian state. West Virginia (WV) has one of the highest rates of diabetes (15.3\%) and pre-diabetes $(35 \%)$ among the 53 US states and territories $[3,4]$. This may be due in part to the majority of the residents residing in rural areas (WV is the 3rd most rural state in the country) [5] and low socioeconomic status, considering that diabetes is highest among individuals who earn a household income of less than $\$ 15,000$ per year, and that $17.9 \%$ of West Virginians are below the poverty level [6]. Other factors that contribute to the high rate of diagnosed and undiagnosed diabetes and pre-diabetes in WV include geography, lack of access to quality care, the aging population, and the Appalachian culture of fatalism [7]. In addition, healthy lifestyle practices are also poor; obesity, physical inactivity, and smoking are major contributing factors to diabetes [1]. Importantly, if not properly managed, dysglycemia (individuals with diabetes and pre-diabetes) can lead to a 
variety of severe complications, including but not limited to heart disease, stroke, hypertension, blindness and eye problems, kidney disease, nervous system disease, and amputation [8]. In WV, diabetes-related diseases such as heart disease, hypertension and stroke are the number 1, 2 and 5 causes of death, respectively [4].

Dysglycemia presents a significant challenge for individuals, communities, and healthcare systems due to increased risk for disease and comorbid conditions associated with health care costs [9-11].

J. Clin. Med. 2018, 7, 58; doi:10.3390/jcm7030058

www.mdpi.com/journal/jcm

Medical expenditure was 2.3 times higher for individuals with diabetes than without and healthcare costs associated with diabetes was $\$ 245$ billion in 2012 [12]. Hence, prevention and education efforts are currently focused on preventing or delaying the early onset of diabetes and its complications. Prevention is one of the most effective public health strategies for reducing the prevalence of diabetes and improving successful disease self-management. Previous evidence-based lifestyle programs, such as the Diabetes Prevention Program (DPP) [13], Da Qing Study [14], Swedish Malmo Study [15], Finnish Diabetes Prevention Study [16], and United Kingdom Prospective Diabetes Study [17], have shown that diet, physical activity, and behavior modifications can combat the rising number of individuals with prediabetes; lifestyle modification is also a critical component of diabetes self-management [18].

While the translation of lifestyle interventions is often recommended for reducing the risk for diabetes and its complications, adequate dissemination of diabetes prevention and management programs in WV (e.g., the DPP, WV Dining with Diabetes program, Chronic Disease Self-Management Program (CDSMP), and Diabetes Self-Management Program (DSMP), and Diabetes Self-Management Education) is less than desired [19]. Understanding the perceived benefits and barriers of participation is critical if we are to improve prevention efforts in WV communities. Rigorous and supportive research on factors that motivate or deter individuals with dysglycemia to participate in lifestyle interventions is currently lacking in this medically underserved state. Furthermore, very little diabetes research has shown adequate dissemination, the use of qualitative techniques, or the understanding of the underlying benefits and barriers involved with lifestyle programming.

Examining the role of benefits and barriers is important in understanding the effectiveness of community-based diabetes programs. Hence, the purpose of this exploratory study was to develop an instrument for assessing the benefits of a community-based diabetes prevention and management (DPM) program. These results can be used to tailor the curriculum for Appalachian culture, and can help with accessibility to diabetes programs in community settings, which may represent a novel way of improving and implementing effective programs in WV. Furthermore, results from this research can be used to tailor new and available programs for improved program adherence, utilization, and behavior changes as well as increase health provider referrals to lifestyle programs in West Virginia communities.

The purpose of the study was to: (1) Determine the participants' perceived barriers for engaging in the DPM program; (2) Determine the benefits of program participation; and (3) Assess the satisfaction with various components of the program (if any).

\section{Experimental Section}

\subsection{Data Source, Eligibility and Recruitment}

The original data source was the Diabetes Prevention and Management (DPM) program from 2014 through 2016. The DPM was implemented in twenty-two sessions over 12 months in two Appalachian churches, and was modeled after the evidence-based National DPP and modified to include diabetes management sessions using the American Association of Diabetes Educators Self-Care Behaviors framework (AADE7) $[13,20]$. The program emphasizes goal-setting and self-monitoring to make lasting improvements in nutrition and physical activity; moderate weight loss; building self-efficacy and social support for sustainable lifestyle changes; and overcoming barriers to maintaining weight-loss and lifestyle changes [21,22]. Specifically, program sessions included, but were not limited to, the following information: healthy eating, physical activity, stress management, keys to eating out, 
mindful eating, problem solving and medication adherence. Program participants could attend all program sessions, and those who missed a session had the opportunity to review a closed YouTube channel that had the program session recordings. The DPM program utilized a prospective study design to evaluate the delivery of an evidencebased, non-pharmacological, behavioral lifestyle intervention. Individuals

who were at risk of developing diabetes (i.e., prediabetes stage) or were diagnosed with diabetes, based on survey screening questions or fasting blood glucose, were recruited for the study. Eligible individuals had to be aged 18 years or older, with a BMI of equal to or greater than $25 \mathrm{~kg} / \mathrm{m}^{2}$, and had to be motivated to meet the Surgeon General's recommended physical activity of at least $150 \mathrm{~min}$ per

week. Potentially eligible participants were recruited from the greater Morgantown and Charleston, WV areas using convenience and snowball sampling due to the nature of the program. These two areas have several hospitals, Federally Qualified Health Centers, free clinics, churches and other community-based organizations (e.g., Young Men's Christian Association) from which participants

were successfully recruited for the program. Participants were also recruited through community advertisements such as flyers, church bulletins, email lists, and community presentations. The study protocol was approved by West Virginia University Institutional Review Board.

\subsection{Benefits and Barriers Survey}

Benefits and barriers were assessed using a 33-item survey questionnaire developed by the researchers. The survey examined participants' perception of benefits of and barriers to participation in the DPM program. The survey questions sought information on the following: barriers to engaging in the program, benefits reported for program participation, satisfaction with various components of the program (if any), and demographic information of the participants (medical history, family history of disease, health literacy, and physical health and mental health wellbeing). In addition, individuals had the opportunity to respond, using open-ended questions, on specific benefits and barriers, provide suggested improvements for the program, and identify the most interesting components that may lead to improvement of community-based diabetes lifestyle programs.

We used a modified Centers for Disease Control clinical data form to identify the medical history of diseases, including prediabetes, diabetes and heart disease [23]. Self-rated general health status was measured by questions modified from the WV Ruby Memorial Hospital Health Questionnaire; the question asked, "Compared to other people, how do you rate your physical health?", with response choices ranging from 1 to 5 , where $1=$ Poor and $5=$ Excellent [24]. Self-rated mental health was measured by the question "Compared to other people, how do you rate your mental health?", with response ranging from 1 to 5 , where $1=$ Poor and $5=$ Excellent. In addition, health literacy was measured by three validated questions developed by Chew et al.: (1) "If you need to go to the doctor, clinic or hospital, how confident are you in filling out forms by yourself?"; (2) "How often do you have someone (family member or staff at the clinic or hospital) help you read health or medical forms?"; and (3) "How often do you have problems learning about your medical condition because of trouble understanding written health information?" $[24,25]$. Response options ranged from 1 to 5 for each question, where $1=$ Not at all confident or Always and $5=$ Extremely confident or Never. Thus, higher scores indicate adequate health literacy.

The remainder of the survey questions were developed and modified from two reliable and valid surveys which were the Community Health Awareness of Diabetes (CHAD) and the Summary of Diabetes Self-Care Activities (SDSCA) measure, along with previous lifestyle programming experience [26,27]. These specific questions identified the benefits and barriers of the diabetes program. The benefits and barriers program questions used Likert response items ( $1=$ Not applicable, $2=$ Strongly Disagree, $3=$ Disagree, $4=$ Neutral, $5=$ Agree, and $6=$ Strongly agree $)$ and an open-ended format. The survey was reviewed by the program staff (Investigators and Health Coaches) to ensure response time, readability, and applicability. Participants were asked to complete the survey without interaction with their peers in order to reduce bias. Confidentiality was assured by using their participant ID number. The questionnaire was completed at 6 months and at the end of the program; however, the last data point was used to assess the participants' perception. All participants were English-speaking individuals and were able to read and 
write in English, a norm in WV. However, study personnel assisted those participants who had questions and/or needed clarification while completing the survey.

\subsection{Statistical Analyses}

Data were analyzed using SPSS (IBM, Armonk, NY, USA) All data was double entered and compared for missingness. While 89 participants (unduplicated) were enrolled in the DPM program, the current analyses included 70 participants with complete data. Descriptive characteristics for the total sample are provided. Cronbach's alpha of this new scale was used to assess reliability or internal consistency, and principal component analysis (PCA) with a varimax rotation was conducted to examine the factorial structure of the constructs, with eigenvalues greater than 1. An eigenvalue of 1 or greater indicates that the factor possesses at least as much total variance as is contained in a single item. PCA was computed by correlating the score for each scale item with the total score for each observation, and by comparing that to the variance for all individual item scores. Model refinement and assessment were carried out iteratively. Following the PCA, findings were quantified and assessed through descriptive analyses. Additionally, the open-ended format for some questions allowed triangulation of the quantitative and qualitative feedback on participants' perceived program benefits and barriers. 3. Results

\subsection{Demographics}

Table 1 presents descriptive statistics on the total sample, which included 89 unduplicated participants. Participants were primarily female (73\%) and aged 20 to 83 years (mean age $=58.51 \pm 0.26$ years). The majority $(62 \%)$ of the participants with diabetes were of low socioeconomic status, had a college degree or higher (69.7\%) and an average income $(45 \%<\$ 50,000)$. Both newly diagnosed and those with longer duration of diabetes were enrolled for the intervention program; the mean number of years with diabetes reported was $19 \pm 13.5$ years, and no significant difference was found between the two groups. Furthermore, two-thirds reported that they were currently married (63\%) and had family members living in their household (mean $=1.24 \pm 0.30$ ).

Table 1. Descriptive statistics of categorical and continuous, demographic variables.

\begin{tabular}{|c|c|c|c|}
\hline Variable & Categories & $n$ & Percent (\%)/Mean \pm SD \\
\hline \multirow{2}{*}{ Sex } & Female & 65 & 73 \\
\hline & Male & 24 & 27 \\
\hline \multirow{2}{*}{ Marital Status } & Not Currently Married & 27 & 30.3 \\
\hline & Currently Married & 56 & 62.9 \\
\hline \multirow{2}{*}{ Education } & High School or Some College & 23 & 25.8 \\
\hline & College Degree or Higher & 62 & 69.7 \\
\hline \multirow{2}{*}{ Income } & $<\$ 50,000$ & 40 & 44.9 \\
\hline & $>\$ 50,000$ & 27 & 30.9 \\
\hline \multirow{2}{*}{ Diabetes Status } & Prediabetes & 34 & 38.2 \\
\hline & Diabetes & 55 & 61.8 \\
\hline Age & & 89 & 58.5 \\
\hline Household Size & & 66 & 1.24 \\
\hline Benefits & & 70 & 5.45 \\
\hline Barriers & & 70 & 4.31 \\
\hline
\end{tabular}

With respect to participants who had a genetic predisposition to diabetes; $64 \%$ indicated a family history, and over half (56\%) knew someone who had been diagnosed with heart disease. Likewise, hypertension (55\%) and high cholesterol (54\%) were major comorbidities for this study sample. 
However, over $50 \%$ of participants self-rated their overall physical and mental health status to be good or excellent, and more than $77 \%$ of participants had adequate health literacy.

Additionally, the rate of program completion for participants was $82 \%$. Dropout was defined as those participants who informed the team that they were withdrawing from the study or when they were unable to be contacted after missing a scheduled assessment and/or follow-up phone calls. Attendance was taken at every educational session. Several individuals started the program, but dropped out in the first 4 weeks due to instrumental reasons such as changes in health and mobility, competing family responsibilities, transportation issues, change in job, etc. Based on attendance, taken at every educational session, the average number of sessions attended was $75 \%$ (or 14 sessions). No significant difference was noted based on diabetes and pre-diabetes status.

\subsection{Reliability of Scale}

PCA identified clustering of five constructs as follows: screening/risk, location, fasting blood work, benefits and barriers. The two factors of importance for this study were benefits and barriers; eight questions loaded for benefits and five questions loaded for barriers. The benefits and barriers components were then placed in a two-component PCA analysis to further assess reliability of the scale, which is presented in Table 2. With the two-component PCA analysis, the overall Cronbach's alpha reliability for the benefits component was 0.827 and 0.645 for barriers. These two factors accounted for 15.3 (benefits) and 21.3 (barriers) of the variance for the total sample. Questions relating to diabetes screening/risk, fasting blood work and location were excluded from the final PCA analysis for this study.

Table 2. Two-factor PCA reliability statistics of benefits and barriers scale.

\begin{tabular}{cccccc}
\hline Variable & Cronbach's Alpha & Mean & Variance & Standard Deviation & N of Items \\
\hline Barriers & 0.645 & 21.5 & 21.3 & 4.62 & 5 \\
Benefits & 0.827 & 43.6 & 15.3 & 3.91 & 8 \\
\hline
\end{tabular}

For the main variables (benefits, barriers), the mean response was $43.6 \pm 3.91$ and $21.5 \pm 4.62$, respectively. The mean scores for these two constructs indicated high benefits and low barriers with the DPM program. DPM program aided in implementing lifestyle changes for participants and educational sessions provided a positive experience for support, discussion and learning opportunities. The benefit item-mean scores ranged from 5.03 to 5.64 on a 1-6 scale (Table 3). Overall, perceived barriers to program participation and engagement was low (range $=3.76$ to 4.69) and therefore included minimal areas of improvement. Most individuals were satisfied with the program and improvement in lifestyle behaviors, and this suggests that individuals rated the individual benefits and barriers items positively. Specifically, individuals with prediabetes (mean $=5.43 \pm 0.528$ ) and diabetes (mean $=5.48$ \pm 0.399 ) scored benefits positively as well.

Table 3. Benefits and barriers items (benefits $n=69$, barriers $n=70$ ).

\begin{tabular}{cccc}
\hline Benefit/Barrier & \multicolumn{1}{c}{ Item } & Mean & Standard Deviation \\
\hline Benefit & $\begin{array}{c}\text { The health coach or program leader } \\
\text { was encouraging or } \\
\text { helpful }\end{array}$ & 5.61 & 0.623 \\
\hline Benefit & $\begin{array}{c}\text { The program } \\
\text { materials/demonstrations were } \\
\text { informative }\end{array}$ & 5.64 & 0.514 \\
\hline Benefit & $\begin{array}{c}\text { I changed my lifestyle as a result of } \\
\text { the program }\end{array}$ & 5.03 & 0.923 \\
\hline Benefit & $\begin{array}{l}\text { I will discuss my program results } \\
\text { with my health provider }\end{array}$ & 5.45 & 0.697 \\
\hline Benefit & $\begin{array}{l}\text { I will discuss my results with my } \\
\text { family and/or friends }\end{array}$ & 5.29 & 0.842 \\
\hline
\end{tabular}




\begin{tabular}{|c|c|c|c|}
\hline Benefit & $\begin{array}{l}\text { The program helped me } \\
\text { understand the risks associated with } \\
\text { diabetes and/or its complications }\end{array}$ & 5.49 & 0.797 \\
\hline Benefit & $\begin{array}{l}\text { The program helped me understand } \\
\text { how to prevent and/or manage } \\
\text { diabetes }\end{array}$ & 5.46 & 0.759 \\
\hline Benefit & Overall, I was satisfied with the program & 5.64 & 0.568 \\
\hline Barrier & Transportation was a barrier for me & 4.69 & 1.27 \\
\hline Barrier & $\begin{array}{l}\text { The weather kept me from attending } \\
\text { some program } \\
\text { sessions }\end{array}$ & 4.33 & 1.40 \\
\hline Barrier & $\begin{array}{l}\text { My health status kept me attending } \\
\text { some program } \\
\text { sessions }\end{array}$ & 4.53 & 1.29 \\
\hline Barrier & $\begin{array}{l}\text { My work kept me from attending } \\
\text { some program } \\
\text { sessions }\end{array}$ & 4.24 & 1.55 \\
\hline Barrier & $\begin{array}{l}\text { Family activities kept me from attending } \\
\text { some program } \\
\text { sessions }\end{array}$ & 3.76 & 1.64 \\
\hline Barrier & $\begin{array}{l}\text { Family activities kept me from attending } \\
\text { some program } \\
\text { sessions }\end{array}$ & 3.76 & 1.64 \\
\hline
\end{tabular}

\subsection{Survey Results}

\subsubsection{Benefits and Barriers}

Over $70 \%$ indicated that the interaction with their personal health coach or program leaders, as well as program materials/demonstrations, were helpful and informative. The program was successful in helping $65 \%$ of individuals to modify their health behavior and make positive lifestyle changes as a result of the program. In addition, $70 \%$ used the results of blood tests completed during the program to discuss with their healthcare provider and family/friends. The majority (74\%) of participants indicated that the program helped them understand the risks associated with dysglycemia and how to prevent/manage diabetes. Overall, about $75 \%$ of respondents were satisfied with the program. Specifically, transportation was not identified as a barrier for the majority of participants. While some educational sessions were done during winter months, over half of the individuals (53\%) reported they did not miss a session due to weather and work priorities, family activities and personal health issues did not deter their attendance.

\subsubsection{Other Constructs}

As mentioned previously, the constructs that were excluded from the two-construct PCA were screening/risk, location and fasting bloodwork. Among these were some interesting conclusions that helped confirm the assumption of high benefits and low barriers. For example, over half $(71 \%, 66 \%)$ of the participants had been screened for diabetes and had been informed of their risk for diabetes by their health provider. Approximately $69 \%$ of respondents indicated that the church location used for program implementation was convenient. Furthermore, fasting blood work done at the churches during weekend mornings was not challenging for approximately $71 \%$ of individuals; over half of participants (54\%) felt comfortable giving blood. The benefits and barriers constructs correlated significantly with participant's self-rated physical health $(r=0.29, p=0.015 \& r=0.366, p=0.002$, 
respectively) indicating individuals with good health perceived greater program benefits and lower barriers to participation. Interestingly, adequate health literacy was not associated with either benefits and barriers of the program.

\subsubsection{Open-Ended Responses}

The themes that emerged related to barriers of the program were health status, work, travel and family activities. To improve the program, the participants suggested to offer more sessions, provide more recipes, more food demonstrations, and guest speakers. Some of the benefits of the program were awareness of food intake, knowledge about disease and tools to control weight, social support and encouragement. Along with the benefits, most participants thought the guest speakers, tracking, and self-motivation presentations were the most interesting parts of the program. Specific benefits and barriers items are provided in Table 4.

Table 4. Benefits and barriers themes from open-ended responses.

\begin{tabular}{cc}
\hline Benefits & Barriers \\
\hline Awareness of disease & Craving at work \\
Encouragement/motivation & Family responsibilities \\
Recipes & Travel activities \\
Lifestyle changes & Church activities \\
Support/relationship building & Health \\
Knowledge & Length of questionnaires \\
Reduction in medications & One meeting time \\
Weight loss & \\
Lower blood pressure & \\
\hline
\end{tabular}

\section{Discussion}

This research study examined the factors that motivated or deterred participants' active participation, as well as suggestions for improvements. Overall, the participants had a positive impression, due to helpful and informative interactions with health coaches and program leaders and improved knowledge and skills for successful lifestyle changes as a result of the program. However, scheduling conflicts with work, travel and family activities were noted as barriers to participation. Therefore, understanding participant perceptions to barriers are critical for addressing concerns for the program.

Health behavior theories cite several factors in behavioral determination that can explain or predict a specific behavior change [28]. An individual's perception can be positive or negative, based on the perceived benefits and barriers before a behavior takes place. For example, an individual will most likely choose a behavior that has the most benefits, especially if the benefits outweigh the barriers [29]. In particular, the health promotion model suggests that engaging in a behavior is dependent on the results of the actions whether it be benefits or barriers. Benefits and barriers can be directly or indirectly motivational to an individual when it comes to behavioral change [30]. Perhaps, directly could be by personal experiences and indirectly might be the result of a previous event. Consequently, prediction of the barriers or costs related to a behavior can in turn affect the intention to engage in a behavior or their level of performance with a behavior [31]. For behavior change, barriers can directly create an obstacle prior to engaging or indirectly through decrease in commitment.

Our results concur with prior studies that highlight the fact that program satisfaction is based on high benefits and low barriers. Interestingly, participants self-rated their health as good or excellent despite their current chronic condition(s) and a genetic predisposition to chronic diseases (based on their family history). Additionally, participants reported adequate literacy levels and socioeconomic status than most areas of WV and were confident in reading and understanding information about their health and disease condition. The program participants were similar in demographic characteristics (age, income, geographical area) to the state, but were more educated ( $70 \%$ 
with Bachelor's degree) and had a higher percent of females [32]. Hence, our study population was representative of the state; the majority of WV counties are rural (ranging from 4-9 in Rural-Urban Commuting Area codes (RUCA)) with our study sample including participants from counties mostly rural (3-6 in RUCA codes) [33].

The program length of one-year was not regarded as a barrier, as has been reported for the National Diabetes Prevention Program, and participants conveyed the satisfaction resulting from peer and program support as they could share their feelings, issues and struggles in a non-judgmental environment, foster peer support and encouragement, and accountability from Health Coaches [34]. The benefits of the long-term program format also allowed for incremental behavioral change and continuous feedback and support for behavior modification. Program location, transportation, weather,

work priorities, and family activities did not pose a barrier and participants were enthusiastic to come for the educational sessions. Given the benefits of the program, $75 \%$ reported their overall satisfaction with participating in the DPM program.

Despite the positive feedback received on the program, there were some limitations with this study. For example, all study variables were self-reported and could have led to measurement error and misclassification bias. Specifically, determination of benefits and barriers scores were based on a self-report measure that was developed for this study. In addition, the study is limited by the small sample size and missingness in the data.

Our findings provide evidence that the DPM program is successful in helping individuals with dysglycemia to make positive lifestyle changes and self-manage their chronic conditions. Hence, the use of Health Coaches and community-based models such as the DPM program can be successful and sustainable in WV. However, while the overall benefits were identified, suggestions for improvements such as to offer more sessions, provide recipes, food demonstrations, and bringing experts can be used to tailor future diabetes prevention and management programs.

\section{Conclusions}

Findings highlight two implications for public health. First, there is a general lack of participant perceptions of community-based diabetes programs, and this study fills a gap in diabetes intervention research in Appalachia, especially in WV. Second, participation in the DPM program improved lifestyle modification while allowing for interventions to become more tailored to the community's needs. By identifying these benefits and barriers, the program effectiveness can be improved resulting in a better quality of life for WV communities.

Acknowledgments: This work was supported by grants from the Eye Foundation of America, Rotary Club International, Claude Worthington Benedum Foundation, Women of WVU, and the WVU Clinical Translational Science Institute. Statistical advice was sought from Christa Lilly of WVU School of Public Health, Department of Biostatistics.

Author Contributions: We declare that we have participated in the manuscript development; and approved the final version. Samantha Shawley-Brzoska and Ranjita Misra helped to develop the study design, acquisition of data, analysis and interpretation of data, critical revision of manuscript for important intellectual content, administrative, technical, or material support, and study supervision. Both authors had full access to the data and take responsibility for the integrity of the data, accuracy of the data analyses and interpretation of the results, and the content in the final submission.

Conflicts of Interest: The authors declare no conflict of interest.

\section{References}

1. Center for Disease Control. National Diabetes Statistics Report; National Center for Chronic Disease Prevention and Health Promotion: Atlanta, GA, USA, 2017.

2. Appalachian Regional Commission. Health Disparities in Appalachia; Appalachian Regional Commission: Washington, DC, USA, 2017.

3. WV Health Statistic Center. West Virginia Behavioral Risk Factor Surveillance System Report. 2013. Available online: http://www.wvdhhr.org/bph/hsc/pubs/brfss/2013/BRFSS2013.pdf (accessed on 15 October 2015). 
4. American Diabetes Association (ADA). The Burden of Diabetes in West Virginia; American Diabetes Association: Arlington County, VA, USA, 2016.

5. The West Virginia Rural Health Association. Health Care in West Virginia: A Workforce Analysis; West Virginia Rural Health Research Center: Morgantown, VA, USA, 2012.

6. The Associated Press. Census Data Show 17.9\% of West Virginians in Poverty. U.S. News, 14 September 2017. 7. Hale, N.L.; Bennett, K.J.; Probst, J.C. Diabetes care and outcomes: Disparities across rural America. J. Community Health 2010, 35, 365374. [CrossRef] [PubMed]

8. Stolar, M. Glycemic control and complications in type 2 diabetes mellitus. Am. J. Med. 2010, 123, S3-S11. [CrossRef] [PubMed]

9. Magid, D.J.; Farmer, S.A. Hypertension self-management: A home run for patients and payers. Circ. Cardiovasc. Qual. Outcomes 2014, 7, 205-206. [CrossRef] [PubMed]

10. Norris, S.L.; Nichols, P.J.; Caspersen, C.J.; Glasgow, R.E.; Engelgau, M.M.; Jack, L.; Snyder, S.R.; Carande-Kulis, V.G.; Isham, G.; Garfield, S.; et al. Increasing diabetes self-management education in community settings. A systematic review. Am. J. Prev. Med. 2002, 22, 39-66. [CrossRef]

11. Uhlig, K.; Patel, K.; Ip, S.; Kitsios, G.D.; Balk, E.M. Self-measured blood pressure monitoring in the management of hypertension: A systematic review and meta-analysis. Ann. Intern. Med. 2013, 159, 185-194. [CrossRef] [PubMed]

12. American Diabetes Association. Economic costs of diabetes in the U.S. in 2012. Diabetes Care 2013, 36, $1033-1046$.

13. Diabetes Prevention Program Research Group. The Diabetes Prevention Program (DPP): Description of lifestyle intervention. Diabetes Care 2002, 25, 2165-2171.

14. Pan, X.R.; Li, G.W.; Hu, Y.H.; Wang, J.X.; Yang, W.Y.; An, Z.X.; Hu, Z.X.; Lin, J.; Xiao, J.Z.; Cao, H.B.; et al.

Effects of diet and exercise in preventing NIDDM in people with impaired glucose tolerance. The Da Qing IGT and diabetes study. Diabetes Care 1997, 20, 537-544. [CrossRef] [PubMed]

15. Eriksson, K.F.; Lindgarde, F. Prevention of type 2 (non-insulin-dependent) diabetes mellitus by diet and physical exercise. The 6-year Malmo feasibility study. Diabetologia 1991, 34, 891-898. [CrossRef] [PubMed]

16. Lindstrom, J.; Louheranta, A.; Mannelin, M.; Rastas, M.; Salminen, V.; Eriksson, J.; Uusitupa, M.; Tuomilehto, J.; Finnish Diabetes Prevention Study Group. The Finnish Diabetes Prevention Study (DPS):

Lifestyle intervention and 3-year results on diet and physical activity. Diabetes Care 2003, 26, 3230-3236. [CrossRef] [PubMed]

17. King, P.; Peacock, I.; Donnelly, R. The UK prospective diabetes study (UKPDS): Clinical and therapeutic implications for type 2 diabetes. Br. J. Clin. Pharmacol. 1999, 48, 643-648. [CrossRef] [PubMed]

18. Rutledge, S.A.; Masalovich, S.; Blacher, R.J.; Saunders, M.M. Diabetes self-management education programs in nonmetropolitan counties-United States, 2016. MMWR Surveill. Summ. 2017, 66, 1-6. [CrossRef] [PubMed]

19. Department of Health and Human Resources. Diabetes Self-Management Programs; Division of Health Promotion and Chronic Disease: Charleston, WV, USA, 2018.

20. AADE7 Self-Care Behaviors. Available online: https://www.diabeteseducator.org/living-with-diabetes/ aade7-self-carebehaviors (accessed on 2 February 2018).

21. Lucke-Wold, B.; Shawley, S.; Ingels, J.S.; Stewart, J.; Misra, R. A critical examination of the use of trained health coaches to decrease the metabolic syndrome for participants of a community-based diabetes prevention and management program. J. Health Commun. 2016, 1, 38. [CrossRef] [PubMed]

22. Ingels, J.S.; Misra, R.; Stewart, J.; Lucke-Wold, B.; Shawley-Brzoska, S. The effect of adherence to dietary tracking on weight loss: Using HLM to model weight loss over time. J. Diabetes Res. 2017, 2017, 6951495. [CrossRef] [PubMed]

23. National Diabetes Prevention Program Curricula and Handouts. Available online: https://www.cdc.gov/ diabetes/prevention/lifestyle-program/curriculum.html (accessed on 2 February 2018).

24. Occupational Medicine Forms. Available online: http://wvumedicine.org/ruby-memorial-hospital/ services/wvu-specialtyclinics/occupational-medicine/forms/ (accessed on 2 February 2018). 
25. Chew, L.D.; Griffin, J.M.; Partin, M.R.; Noorbaloochi, S.; Grill, J.P.; Snyder, A.; Bradley, K.A.; Nugent, S.M.; Baines, A.D.; VanRyn, M. Validation of screening questions for limited health literacy in a large VA outpatient population. J. Gen. Intern. Med. 2008, 23, 561-566. [CrossRef] [PubMed]

26. Toobert, D.J.; Hampson, S.E.; Glasgow, R.E. The summary of diabetes self-care activities measure: Results from 7 studies and a revised scale. Diabetes Care 2000, 23, 943-950. [CrossRef] [PubMed]

27. Agarwal, G.; Kaczorowski, J.; Hanna, S. Community health awareness of diabetes (CHAD): Description of a community-wide diabetes awareness demonstration program and its feasibility. Can. J. Diabetes 2013, 37, 294-300. [CrossRef] [PubMed]

28. Glanz, K.; Rimer, B.; Viswanath, K. Health Behavior and Health Education: Theory, Research, and Practice, 4th ed.; JosseyBass: San Francisco, CA, USA, 2008; 583p.

29. Sharifirad, G.; Mohebi, S.; Azadbakht, L.; Feizi, A.; Kargar, M. Structural role of perceived benefits and barriers to self-care in patients with diabetes. J. Educ. Health Promot. 2013, 2, 37. [CrossRef] [PubMed]

30. Pender, N.J.; Murdaugh, C.L.; Parsons, M.A. Health Promotion in Nursing Practice, 4th ed.; Prentice Hall: Upper Saddle River, NJ, USA, 2002; p. 60.

31. McEvoy, P.M.; Nathan, P. Perceived costs and benefits of behavioral change: Reconsidering the value of ambivalence for psychotherapy outcomes. J. Clin. Psychol. 2007, 63, 1217-1229. [CrossRef] [PubMed] 32. U.S. Census Bureau QuickFacts: West Virginia. Available online: https://www.census.gov/quickfacts/WV (accessed on 2 February 2018).

33. Rural-Urban Continuum Codes. United States Department of Agriculture Economic Research Service. Available online: https://www.ers.usda.gov/data-products/rural-urban-continuum-codes/ (accessed on 2 February 2018).

34. Johnson, L.A.; Melton, S.T. Perceived benefits and barriers to the diabetes prevention program. PLAID 2016, 2, 16-24. [CrossRef]

(C) 2018 by the authors. Licensee MDPI, Basel, Switzerland. This article is an open access article distributed under the terms and conditions of the Creative Commons Attribution (CC BY) license (http://creativecommons.org/licenses/by/4.0/). 


\section{Appendix D}

\begin{tabular}{|c|c|c|c|c|c|c|c|c|c|c|c|}
\hline \multicolumn{12}{|c|}{ Bivariate Correlation Matrix } \\
\hline & 1 & 2 & 3 & 4 & 5 & 6 & 7 & 8 & 9 & 10 & 11 \\
\hline 1. Benefits & - & & & & & & & & & & \\
\hline 2. Barriers & -0.027 & - & & & & & & & & & \\
\hline 3. Age & -0.024 & $.275^{*}$ & - & & & & & & & & \\
\hline 4. Marital Status & -0.079 & -0.054 & $-.238^{*}$ & - & & & & & & & \\
\hline 5. Education & -0.060 & -0.130 & -0.132 & -0.014 & - & & & & & & \\
\hline 6. Program Attendance & 0.232 & $.454^{* *}$ & $.325^{* *}$ & -0.160 & -0.069 & - & & & & & \\
\hline 7. Percent Body Weight & -0.142 & 0.104 & 0.011 & -0.096 & -0.061 & $-.251^{*}$ & - & & & & \\
\hline 8. Income & $-.266^{*}$ & -0.058 & -0.163 & $.246^{*}$ & $.317^{* *}$ & -0.205 & -0.077 & - & & & \\
\hline 9. Diabetes Status & -0.046 & -0.157 & $.223^{*}$ & -0.164 & -0.065 & 0.056 & 0.000 & -0.047 & - & & \\
\hline 10. Household Size & $-.248^{*}$ & -0.091 & -0.153 & $.377^{* *}$ & 0.004 & -0.099 & -0.190 & 0.181 & 0.052 & - & \\
\hline 11. Gender & -0.051 & 0.145 & 0.008 & 0.108 & 0.058 & -0.100 & 0.166 & 0.164 & -0.095 & -0.161 & - \\
\hline
\end{tabular}

$* \mathrm{p}<0.05, * * \mathrm{p}<0.01$ 
Appendix E

\section{Baron \& Kenny Testing}

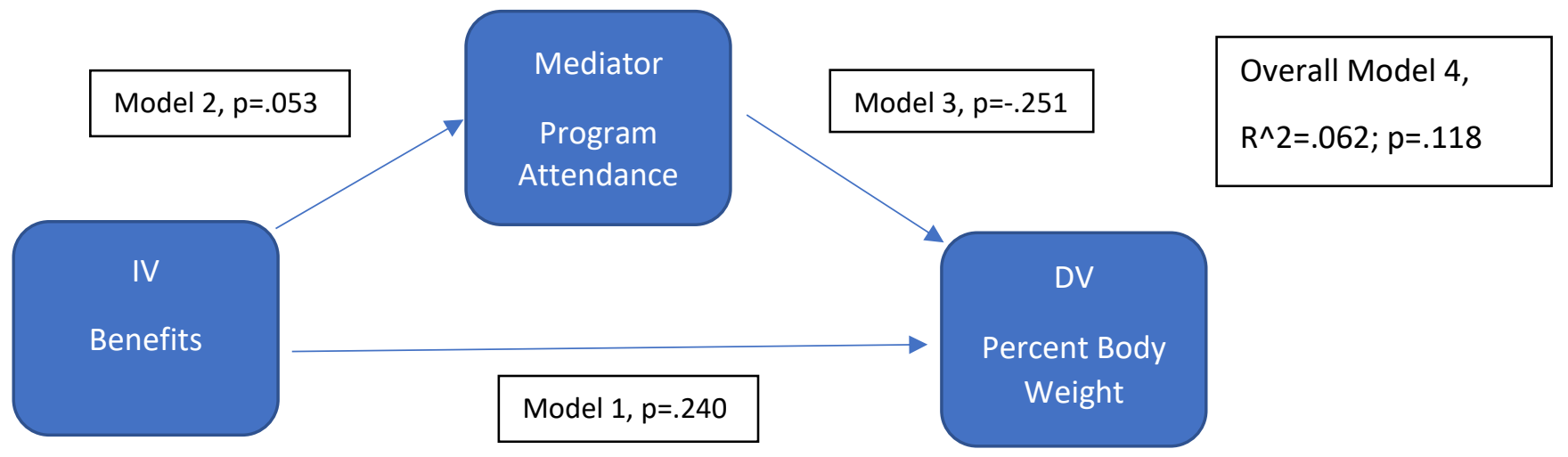

\begin{tabular}{|l|l|}
\hline Steps/Models & Equation \\
\hline 1 & Benefits $\rightarrow$ Percent Body Weight \\
\hline 2 & Benefits $\rightarrow$ Program Attendance \\
\hline 3 & Program Attendance $\rightarrow$ Percent Body Weight \\
\hline 4 & $\begin{array}{l}\text { Benefits Percent Body Weight } \\
\text { Program Attendance }\end{array}$ \\
\hline
\end{tabular}

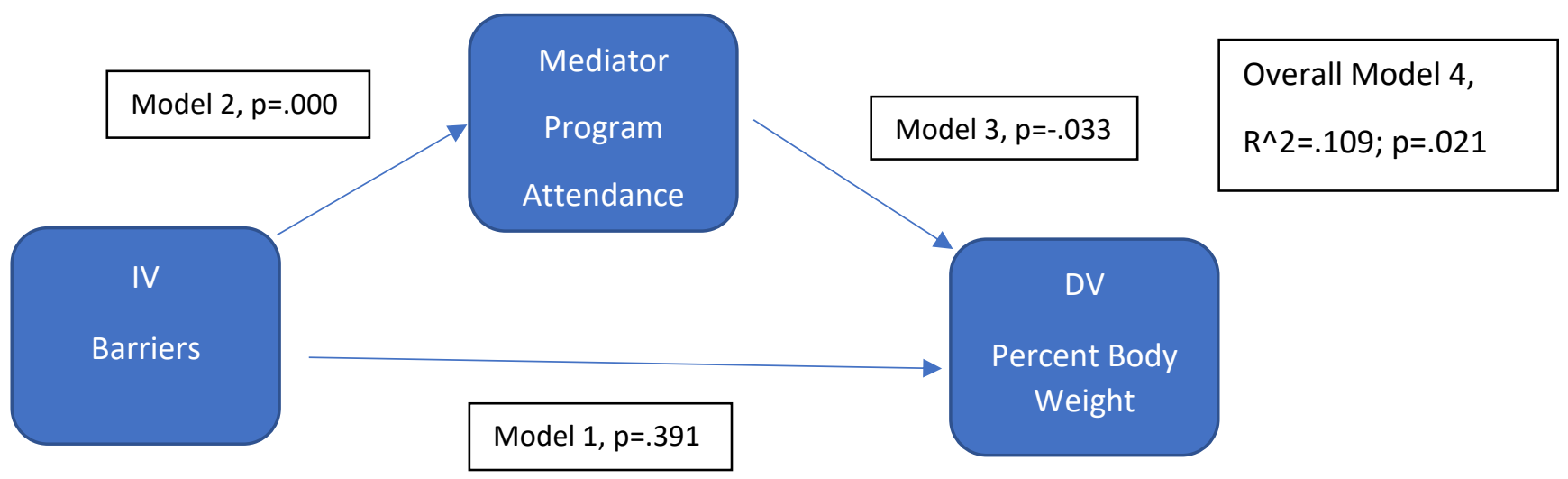

\begin{tabular}{|l|l|}
\hline Steps/Models & Equation \\
\hline 1 & Barriers $\rightarrow$ Percent Body Weight \\
\hline 2 & Barriers $\rightarrow$ Program Attendance \\
\hline 3 & $\begin{array}{l}\text { Program Attendance } \rightarrow \text { Percent Body } \\
\text { Weight }\end{array}$ \\
\hline 4 & $\begin{array}{l}\text { Barriers Percent Body Weight } \\
\text { Program Attendance }\end{array}$ \\
\hline
\end{tabular}




\section{Appendix F}

Baron and Kenny Sensitivity Analysis

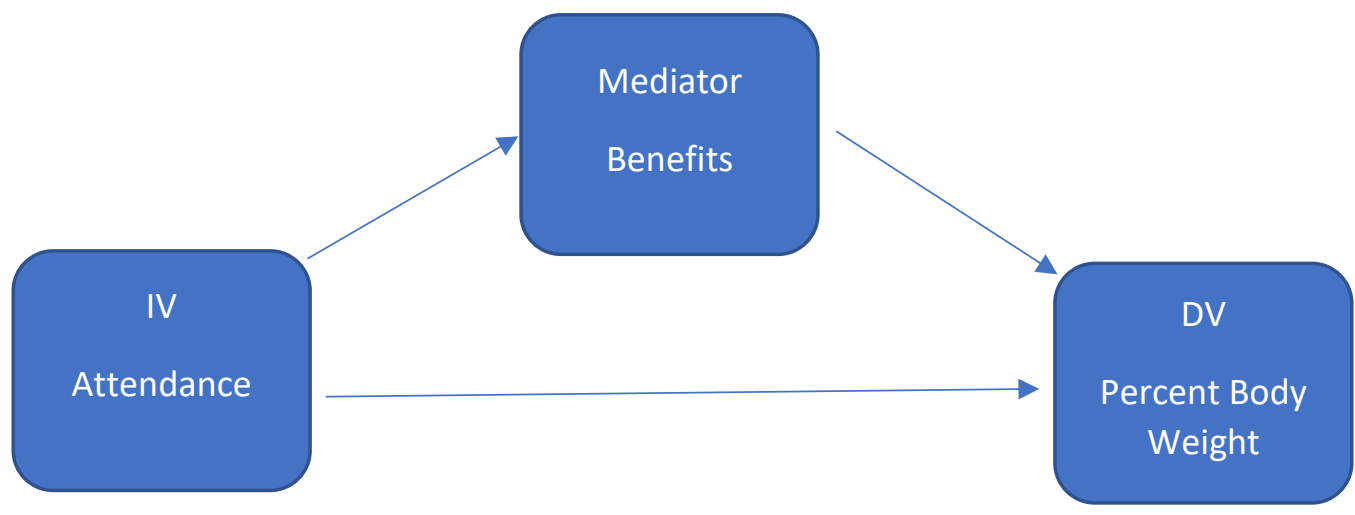

\begin{tabular}{|l|l|l|l|}
\hline Benefits & \multicolumn{3}{l|}{} \\
\hline Steps/Models & Equation & Standardized B & p-value \\
\hline 1 & $\mathrm{X} \rightarrow \mathrm{Y}$ & -0.251 & 0.033 \\
\hline 2 & $\mathrm{X} \rightarrow \mathrm{M}$ & 0.232 & 0.053 \\
\hline 3 & $\mathrm{M} \rightarrow \mathrm{Y}$ & -0.142 & 0.240 \\
\hline 4 & $\mathrm{X} \rightarrow \mathrm{Y}$ & -0.210 & 0.089 \\
& $\mathrm{M}$ & -0.094 & 0.444 \\
\hline
\end{tabular}

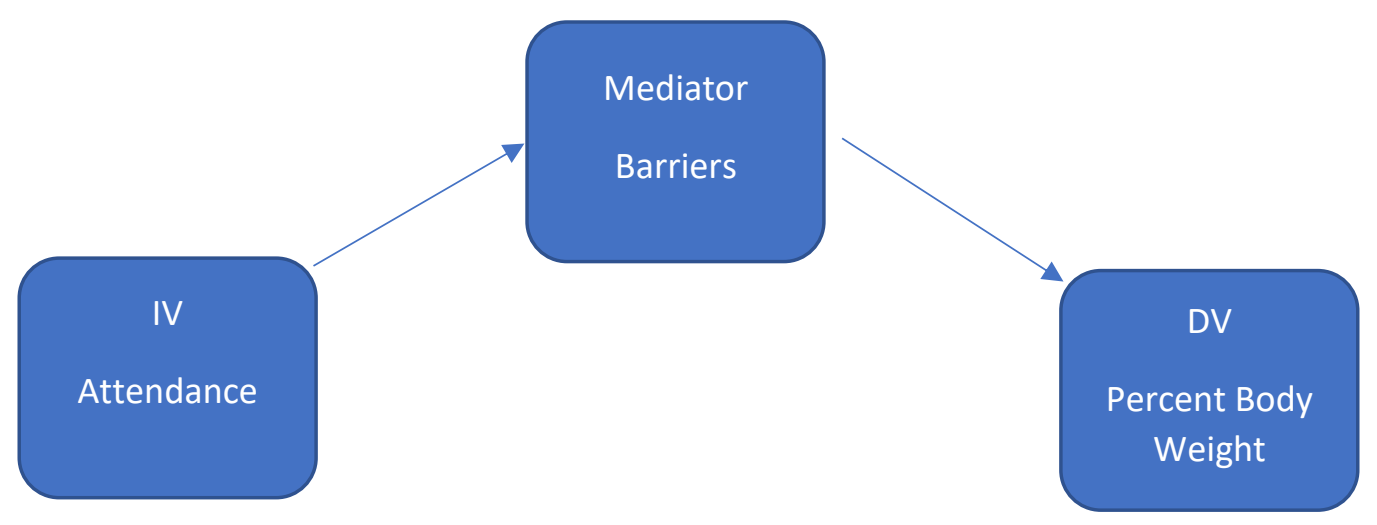

\begin{tabular}{|l|l|l|l|}
\hline Barriers & \multicolumn{3}{l|}{} \\
\hline Steps/Models & Equation & Standardized B & p-value \\
\hline 1 & $\mathrm{X} \rightarrow \mathrm{Y}$ & -0.251 & 0.033 \\
\hline 2 & $\mathrm{X} \rightarrow \mathrm{M}$ & 0.454 & 0.000 \\
\hline 3 & $\mathrm{M} \rightarrow \mathrm{Y}$ & 0.104 & 0.391 \\
\hline 4 & $\mathrm{X} \rightarrow \mathrm{Y}$ & -0.351 & 0.008 \\
& $\mathrm{M}$ & 0.254 & 0.046 \\
\hline
\end{tabular}




\section{Appendix G}

\section{SOCIAL SUPPORT AND EATING HABITS SURVEY}

Below is a list of things people might do or say to someone who is trying to improve their eating habits. We are interested in high fat and high salt (or high sodium) foods. If you are not trying to make any of these dietary changes, then some of the questions may not apply to you, but please read and give an answer to every question.

Please rate each question twice. Under family, rate how often anyone living in your household has said or done what is described during the last three months. Under friends, rate how often your friends, acquaintances, or coworkers have said or done what is described during the last three months.

Please write one number from the following rating scale in each space:

SAMPLE:

A. If my family rarely makes fun of the foods I eat, and my friends very often do. Family Friends I would answer like this:

A. Made fun of the foods I eat

A. 2 A. 5

\begin{tabular}{cccccc} 
none & rarely & $\begin{array}{c}\text { a } \\
\text { few } \\
\text { times }\end{array}$ & often & $\begin{array}{c}\text { very } \\
\text { often }\end{array}$ & $\begin{array}{c}\text { does } \\
\text { not } \\
\text { apply }\end{array}$ \\
\hline 1 & 2 & 3 & 4 & 5 & 8
\end{tabular}

During the past three months, my family (or members of my household) or friends:

1. Encouraged me not to eat "unhealthy foods" (cake, salted chips) when I'm tempted to do so.

2. Discussed my eating habit. changes with me (asked me how I'm doing with my eating changes).

3. Reminded me not to eat high fat, high salt foods.

\begin{tabular}{ll} 
Family & Friends \\
\hline 1. & 1. \\
\hline 2. & 2. \\
\hline 3. & 3. \\
\hline 4. & 4. \\
\hline 5. & 5. \\
\hline 6. & 6. \\
\hline Z. & 7. \\
\hline 8. & 8. \\
\hline 9. & 9. \\
\hline 10. & 10. \\
\hline
\end{tabular}




\section{Appendix H}

\section{SOCIAL SUPPORT AND EXERCISE SURVEY}

Below is a list of things people might do or say to someone who is trying to exercise regularly. If you are not trying to exercise, then some of the questions may not apply to you, but please read and give an answer to every question.

Please rate each question twice. Under family, rate how often anyone living in your household has said or done what is described during the last three months. Under friends, rate how often your friends, acquaintances, or coworkers have said or done what is described during the last three months.

Please write one number from the following rating scale in each space:

\begin{tabular}{|c|c|c|c|c|c|}
\hline none & rarely & $\begin{array}{c}\text { a } \\
\text { few } \\
\text { times }\end{array}$ & often & $\begin{array}{l}\text { very } \\
\text { often }\end{array}$ & $\begin{array}{l}\text { does } \\
\text { not } \\
\text { apply }\end{array}$ \\
\hline 1 & 2 & 3 & 4 & 5 & 8 \\
\hline
\end{tabular}

During the past three months, my family (or members of my household) or friends:

11. Exercised with me.

12. Offered to exercise with me.

13. Gave me helpful reminders to exercise ("Are you going to exercise tonight? ).

14. Gave me encouragement. to stick with my exercise program.

15. Changed their schedule so we could exercise together.

16. Discussed exercise with me.

17. Complained about the time I spend exercising.

18. Criticized me or made fun of me for exercising.

19. Gave me rewards for exercising (bought me something or gave me something I like).

20. Planned for exercise on recreational outings.

21. Helped plan activities around my exercise.

22. Asked me for ideas on how they can get more exercise.

23. Talked about how much they like to exercise.
Family Friends

$\frac{11 .}{12 .}$

$14 . \quad 14$.

15.15

16.

$17 . \quad 17$

$18 . \quad 18$.

$19 . \quad 19$

20.

21.21

$22 . \quad 22$

23.23

$\square$ 1. English $\square$ 2. Spanish Date: Entered

Office Use Only 


\section{Appendix I}

\section{SDSU JOINT DOCTORAL PROGRAM IN CLINICAL PSYCHOLOGY UCSD}

October 1996

TO: Users of Social Support Surveys for Diet and Exercise Behaviors

FROM: James F. Sallis, $\mathrm{Ph} . \mathrm{D}$.

RE: $\quad$ Scoring of scales

Enclosed are copies of abbreviated versions of the Social Support and Eating Habits Survey and Social Support and Exercise Survey. These were designed to be easier to use than the original, complete scales reported in Preventive-Medicine.

In sconing either the complete or abbreviated scales "8" should be recoded to "1."

The abbreviated Social Support for Eating Habits Survey should be scored separately for family and finends.

Encouragement: sum items 1 -5

Discouragement: sum items $6-10$

The Social Support and Exercise Survey should be scored differently for friends and family.

Family Participation: sum items $11-16$ and $20-23$

Family Rewards and Punishment (an optional scale): sum items 17 - 19

Friend Participation: sum items $11-16$ and $20-23$

The Rewards and Punishment subscale should not be scored for friends because it did not emerge in the factor analysis.

Reference:

Sallis, J.F., Grossman, R.M., Pinski, R B., Patterson, T. L. and Nader, P.R. (1987).

The development of scales to measure social support for diet and exercise behaviors.

Preventive Medicine, 16, 825-836.

Address:

6363 Alvarado Court, Suite 250

San Diego, CA 92120

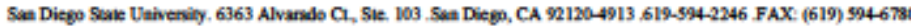
Univenily of Califomia, San Dicge. 3427 Fourth Ave. San Diega. CA 92103 , 619-497-6659. FAX (619) 497-6696 


\section{Appendix J}

\begin{tabular}{|c|c|c|c|c|c|c|c|c|c|c|c|c|c|c|c|c|c|c|}
\hline \multicolumn{19}{|c|}{ Bivariate Correlation Matrix } \\
\hline & 1 & 2 & 3 & 4 & 5 & 6 & 7 & 8 & 9 & 10 & 11 & 12 & 13 & 14 & 15 & 16 & 17 & 18 \\
\hline 1. HDL Change & - & & & & & & & & & & & & & & & & & \\
\hline 2. A1c Change & -0.103 & - & & & & & & & & & & & & & & & & \\
\hline 3. LDL Change & -0.083 & 0.074 & - & & & & & & & & & & & & & & & \\
\hline 4. Program Attendance & -0.080 & -0.260 & 0.157 & - & & & & & & & & & & & & & & \\
\hline 5. Age & -0.114 & -0.104 & 0.103 & $.323^{* *}$ & - & & & & & & & & & & & & & \\
\hline 6. Gender & -0.056 & 0.015 & $-.336^{*}$ & 0.087 & 0.004 & - & & & & & & & & & & & & \\
\hline 7. Marital Status & -0.229 & -0.273 & -0.001 & -0.053 & -0.151 & 0.157 & - & & & & & & & & & & & \\
\hline 8. Education & 0.148 & 0.172 & $-.411^{*}$ & -0.006 & -0.198 & 0.058 & -0.020 & - & & & & & & & & & & \\
\hline 9. Diabetes Status & 0.262 & 0.084 & 0.106 & 0.035 & 0.166 & -0.138 & -0.179 & -0.146 & - & & & & & & & & & \\
\hline 10. Household Size & 0.001 & 0.009 & -0.073 & -0.089 & -0.071 & -0.158 & $.301^{*}$ & -0.006 & 0.020 & - & & & & & & & & \\
\hline 11. Income & -0.089 & 0.097 & -0.134 & -0.258 & -0.159 & 0.176 & 0.259 & $.290^{*}$ & -0.087 & 0.154 & - & & & & & & & \\
\hline 12. Encouragement Family & 0.036 & 0.222 & -0.206 & -0.124 & -0.128 & $.368^{* *}$ & 0.223 & 0.128 & -0.134 & 0.049 & 0.225 & - & & & & & & \\
\hline 13. Discouragement Family & 0.135 & -0.038 & -0.206 & -0.081 & -0.144 & 0.007 & $.275^{*}$ & $.301^{*}$ & -0.048 & $.340^{*}$ & -0.101 & 0.038 & - & & & & & \\
\hline 14. Discouragement Friends & 0.176 & 0.107 & -0.045 & -0.191 & -0.095 & -0.156 & -0.171 & $.307^{*}$ & 0.079 & -0.204 & -0.101 & $.299^{*}$ & 0.131 & - & & & & \\
\hline 15. Encouragement Friends & 0.217 & 0.115 & -0.182 & $-.342^{* *}$ & -0.067 & 0.052 & -0.110 & $.286^{*}$ & 0.217 & -0.109 & -0.073 & 0.171 & $.483^{* *}$ & $.455^{* *}$ & - & & & \\
\hline 16. Family Participation & -0.245 & 0.056 & -0.229 & -0.128 & -0.131 & 0.182 & 0.227 & 0.140 & -0.026 & 0.074 & 0.041 & $.565^{* *}$ & -0.075 & 0.128 & -0.108 & - & & \\
\hline 17. Friend Participation & 0.158 & 0.036 & 0.118 & -0.132 & -0.174 & -0.037 & -0.092 & 0.173 & 0.197 & -0.099 & -0.042 & 0.186 & -0.037 & $.575^{* *}$ & 0.125 & 0.208 & - & \\
\hline 18. Family Rewards Punishment & $-.306^{*}$ & 0.102 & -0.178 & -0.025 & -0.128 & 0.198 & 0.247 & 0.026 & 0.016 & 0.013 & 0.024 & $.483^{* *}$ & -0.056 & 0.235 & -0.092 & $.705^{* *}$ & $.383^{* *}$ & - \\
\hline
\end{tabular}




\section{Appendix K}

\section{WestVurginiaUniversity.}

Dear Participant,

This is a request to participate in a moderated focus group to evaluate your feedback of the Diabetes Prevention and Management Program. Responses will be utilized by health coaches (Meredith Brown, Ben Frear, Samantha Shawley, Jay Stewart, Kara Viggiano, and Megan Whetzel) to aid in making modifications and improvements to future programs. It will be supervised by Dr. Ranjita Misra, Professor, Department of Social and Behavioral Sciences, WVU School of Public Health.

The focus group will last approximately $60-90$ minutes. Open-ended questions/prompts will be provided in a discussion-type format. It will be facilitated by Delores James, Associate Professor, Department of Health Education and Behavior, University of Florida College of Health and Human Performance, in an effort to minimize bias. You will not be required to answer each question; you may withdraw at any time without consequence. Responses will be audiorecorded, but kept confidential. Please do not provide any identifying information during the discussion. Anonymity cannot be guaranteed due to audio recording of focus group. There are no anticipated risks involved. You will receive a $\$ 20$ gift card for participating.

If you have any further questions or concerns about participation in the focus group, please contact Dr. Ranjita Misra at (304)293-4168 or by e-mail at ramisra@hsc.wvu.edu.

Thank you for your time and participation.

Sincerely,

Dr. Ranjita Misra

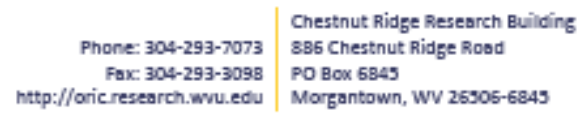




\section{Appendix L}

\section{Translating Diabetes Prevention and Management into Appalachian Communities Focus Group Guide}

Time of Interview: 60-90 Minutes

Place: Woodland Methodist Church/Village Chapel Presbyterian Church

Moderator/Consultant: Danielle Davidov

Focus Group Participants: Participants

\section{Introduction/Purpose:}

Hello! How are you doing today?

My name is Danielle Davidov. I am an Assistant Professor in the Department of Emergency Medicine and Social and Behavioral Sciences at West Virginia University. We have an IRB on file for this project. Confidentiality will be maintained throughout the focus group process. You may choose to end the conversation or skip a question at any time. Anonymity cannot be guaranteed due to audio recording of focus group. Thank you for agreeing to take some time out of your busy schedule to meet with me to discuss your experiences and perspectives related to the Diabetes Prevention and Management Program. To better facilitate note-taking and evaluation, I am audiotaping our conversation today.

I am going to go ahead and begin asking questions. Is that okay with you?

\section{Focus Group Questions}

\section{Core Questions}

1. What comes to mind when you think of diabetes?

a. Probe 1. How has your view of diabetes changed since you participated in the program?

2. Why did you decide to be involved with the diabetes program?

a. Probe 1. What did you want to get out of the program?

i. Were those needs met? 
1. If not, how could they have been met?

3. What challenges did you experience with the program?

a. Probe 1. What barriers (if any) made it difficult for you to attend the sessions?

i. What about transportation, time the sessions were scheduled, weather, etc.?

b. Probe 2. What could the program have done to minimize those challenges?

4. What changes (if any) would you like to see in the program?

a. Probe 1. What about the day, time, and location?

b. Probe 2. Were the meeting times too frequent or not frequent enough?

c. Probe 3. Were the incentives or payment adequate?

5. Do you have any other comments or suggestions on how we can modify or improve the program?

\section{Group Specific Questions}

6. Please describe your overall experience in participating in the program.

a. Probe 1. How has the program helped you?

i. How has your diet changed?

ii. How has your activity level changed?

iii. How has your weight changed?

b. Probe 2. Is there anything that has improved in your life other than your eating and activity level?

7. What kept you motivated to keep attending the sessions?

a. Probe 1. What about a sense of obligation, family support, or new friendships within the program?

8. What are three useful things that you have learned from the program?

a. Probe 1. How are you using or practicing them?

9. Please describe your experience of the weekly contact with your health coach?

a. Probe 1 . Were there any things that were annoying? If so, what?

b. Probe 2. What are some of the biggest ways that the coach helped? 
10. Please describe your experience in completing the tracking booklets each week?

a. Probe 1. What was the biggest challenge? 


\section{Appendix M}

\section{Dissertation Aim 3}

\section{Qualitative Data Analysis Codebook}

\section{Nodes}

\begin{tabular}{|c|c|}
\hline Name & Description \\
\hline Barriers & $\begin{array}{l}\text { A negative item, activity or action that could hinder } \\
\text { engagement/participation in healthy behavior(s) and/or the } \\
\text { diabetes program }\end{array}$ \\
\hline Decreased Program Participation & $\begin{array}{l}\text { A negative item, activity or action that could decrease } \\
\text { engagement/participation in the diabetes program }\end{array}$ \\
\hline Attitude & Personality trait that impacts program success for participants \\
\hline Being Alone & $\begin{array}{l}\text { Having only yourself to rely on, feeling isolated and separate from } \\
\text { others }\end{array}$ \\
\hline Care Giving & Spending time to take care of others to improve their health \\
\hline Audience Appropriateness & $\begin{array}{l}\text { A need for speakers to be of the appropriate demographics for the } \\
\text { audience as well as a more diverse group of program participants }\end{array}$ \\
\hline Bloodwork & $\begin{array}{l}\text { Issues were raised for the initial bloodwork such as qualifications of } \\
\text { phlebotomists and completing the blood draw }\end{array}$ \\
\hline $\begin{array}{l}\text { Diabetes Status and } \\
\text { Knowledge }\end{array}$ & $\begin{array}{l}\text { Disease status of the participants related to diabetes and } \\
\text { knowledge of the disease, risk factors and complications }\end{array}$ \\
\hline $\begin{array}{l}\text { Health Status/Clinical } \\
\text { Outcomes }\end{array}$ & $\begin{array}{l}\text { Health alerts, indicators and issues from diabetes and other } \\
\text { complications }\end{array}$ \\
\hline $\begin{array}{l}\text { Frequency and Organization } \\
\text { of Meetings }\end{array}$ & Meeting logistics related to occurrence and timing meetings \\
\hline Health Coach & $\begin{array}{l}\text { Experience with assigned health coach and suggestions for } \\
\text { improvements on matching, consistency of communication and } \\
\text { overall one on one relationship }\end{array}$ \\
\hline Program Logistics & Issues with physical workflow of program \\
\hline
\end{tabular}




\begin{tabular}{|c|c|}
\hline Name & Description \\
\hline Incentives & $\begin{array}{l}\text { Thoughts on payments given to participants for their participation } \\
\text { in the program }\end{array}$ \\
\hline $\begin{array}{l}\text { Items that weren't } \\
\text { addressed }\end{array}$ & Examples of items not sought out during the program \\
\hline Program Ending & $\begin{array}{l}\text { Items the participants are worried about when the program comes } \\
\text { to an end }\end{array}$ \\
\hline Video & Issues with the technology and sound effects for session videos \\
\hline Tracking & $\begin{array}{l}\text { Experience with keeping track of nutritional values and physical } \\
\text { activity }\end{array}$ \\
\hline Unhealthy Behavior & Behaviors that hinder positive health outcomes \\
\hline Food Addiction & $\begin{array}{l}\text { Dependent on specific food item(s) and inability to stop eating even } \\
\text { with future consequences }\end{array}$ \\
\hline Exercising & Difficulties with engaging in physical activity and being active \\
\hline Previous Unhealthy Ways & $\begin{array}{l}\text { Previous exposure to unhealthy habits related to diabetes } \\
\text { complications, physical activity and diet }\end{array}$ \\
\hline Unhealthy Eating & Diet habits that may hinder health outcomes and/or goals \\
\hline $\begin{array}{l}\text { Eating different than } \\
\text { others }\end{array}$ & $\begin{array}{l}\text { Diet patterns that can be different than friends, peers and/or family } \\
\text { including spouse }\end{array}$ \\
\hline Eating Out & $\begin{array}{l}\text { Habits and issues related to eating a meal in the restaurant or on- } \\
\text { the-go }\end{array}$ \\
\hline Meals & Issues with managing meal decisions on evenings and weekends \\
\hline Weight Loss & $\begin{array}{l}\text { Issues with decreasing weight due to chronic conditions, ongoing } \\
\text { health habits and health status }\end{array}$ \\
\hline Benefits & $\begin{array}{l}\text { A positive item, activity or action that results in promoting healthy } \\
\text { behavior(s) and/or diabetes program participation }\end{array}$ \\
\hline Enhanced Program Participation & $\begin{array}{l}\text { A positive item, activity or action that could decrease } \\
\text { engagement/participation in the diabetes program }\end{array}$ \\
\hline Camaraderie and Support & Collaborative and supportive atmosphere among peers \\
\hline
\end{tabular}




\begin{tabular}{|c|c|}
\hline Name & Description \\
\hline Commitment & Dedication and devotion to participating in program \\
\hline $\begin{array}{l}\text { Health Coaches and } \\
\text { Program Leaders }\end{array}$ & Experience with health coaches and/or program leaders \\
\hline Guest Lectures & $\begin{array}{l}\text { Experience with speakers coming in to present new topics and/or } \\
\text { activities }\end{array}$ \\
\hline $\begin{array}{l}\text { Knowledge, Acceptance and } \\
\text { Control }\end{array}$ & $\begin{array}{l}\text { Accepting, learning and controlling your needs and wants when } \\
\text { striving to better your health and well-being }\end{array}$ \\
\hline Program Logistics & Experience with physical workflow of program \\
\hline Frequency of meetings & Meeting logistics related to occurrence and timing meetings \\
\hline Location & Setting/area that the program sessions took place \\
\hline Incentives & $\begin{array}{l}\text { Thoughts on payments given to participants for their participation } \\
\text { in the program }\end{array}$ \\
\hline Video & Feedback on the technology and sound effects for session videos \\
\hline Program Success & Overall success related to sessions, outcomes and experience \\
\hline Tracking & $\begin{array}{l}\text { Experience with keeping track of nutritional values and physical } \\
\text { activity }\end{array}$ \\
\hline Health & Improvement of health through behavior change \\
\hline Health Outcomes & Experience related to improvement of health outcomes \\
\hline Bloodwork & $\begin{array}{l}\text { Thoughts on completing the blood draw and change in health } \\
\text { outcomes based on bloodwork values }\end{array}$ \\
\hline Medications & Medication regime changes based on program \\
\hline Overall Health & $\begin{array}{l}\text { Feedback on overall health improvements from participating in the } \\
\text { program }\end{array}$ \\
\hline
\end{tabular}




\begin{tabular}{|c|c|}
\hline Name & Description \\
\hline Weight Loss & Thoughts on weight loss from participating in the program \\
\hline Healthy Behavior & Behaviors that result in positive health outcomes \\
\hline Exercising & Improvements and gains with engaging in physical activity regularly \\
\hline Healthy Eating & Diet habits that may improve health outcomes and/or goals \\
\hline Social Support & $\begin{array}{l}\text { Aid/assistance occurring within the context of a social network, } \\
\text { which could be influences and interactions with family, friends, } \\
\text { peers and other in the community }\end{array}$ \\
\hline Family & $\begin{array}{l}\text { Support received from family members on program participation } \\
\text { and healthy lifestyle }\end{array}$ \\
\hline Family History of Diabetes & History of diabetes, the risk factors involved and complications \\
\hline Friends-Coworkers & $\begin{array}{l}\text { Support received from friends and/or coworkers on program } \\
\text { participation and healthy lifestyle }\end{array}$ \\
\hline $\begin{array}{l}\text { Health Coach and Program } \\
\text { Leaders }\end{array}$ & $\begin{array}{l}\text { Support received and relationship with health coaches and/or } \\
\text { program leaders }\end{array}$ \\
\hline Peers-Fellow Participants & $\begin{array}{l}\text { Support received from peers and/or fellow participants on program } \\
\text { participation and healthy lifestyle }\end{array}$ \\
\hline Yourself & $\begin{array}{l}\text { Motivation for yourself on program participation and healthy } \\
\text { lifestyle }\end{array}$ \\
\hline
\end{tabular}




\section{Appendix N}

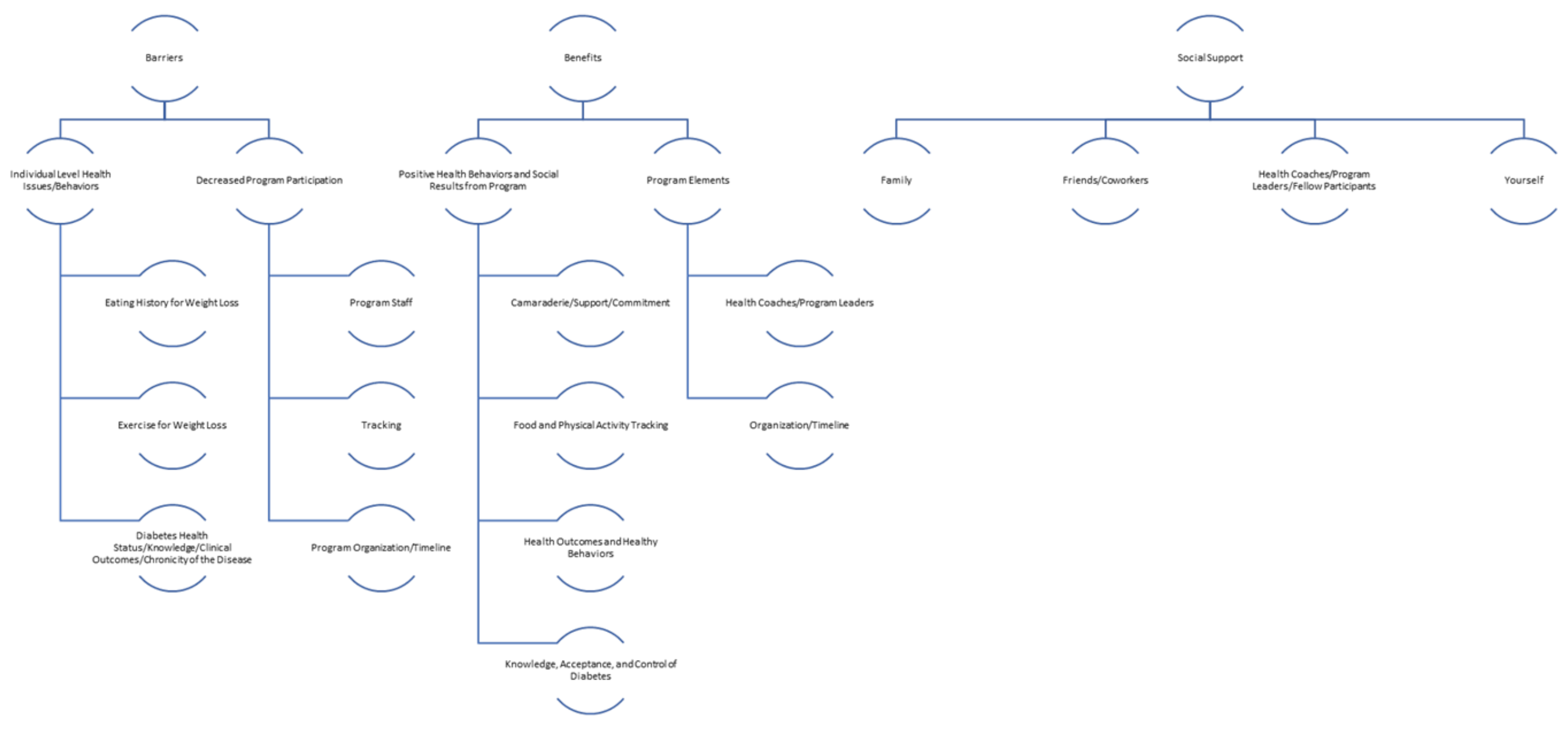


Appendix O

\section{Barriers}

Individual Level Health Issues/Behaviors

Eating History for Weight Loss

Exercise for Weight Loss

Diabetes Health Status/Knowledge/Clinical Outcomes/Chronicity of the Disease

Program Staff

Decreased Program Participation

Tracking

Program Organization/Timeline

Camaraderie/Support/Commitment

Benefits

Positive Health Behaviors and Social Results from

Food and Physical Activity Tracking

Program

Health Outcomes and Healthy Behaviors

Knowledge, Acceptance, and Control of Diabetes

Program Elements

Health Coaches/Program Leaders

Organization/Timeline

\section{Social \\ Support \\ Family \\ Friends/Coworkers \\ Health Coaches/Program Leaders/Fellow Participants}

Yourself 


\section{References}

AADE. (2008). AADE7 self-care behaviors. The Diabetes Educator, 34(3), 445-9.

Ackermann, R.T., Finch, E.A., Brizendine, E., Zhou, H., \& Marrero, D.G. (2008). Translating the diabetes prevention program into the community: The deploy pilot study. American Journal of Preventive Medicine, 35(4), 357-363.

Ackermann, R.T., \& Marrero, D.G. (2007). Adapting the diabetes prevention program lifestyle intervention for delivery in the community. The ymca model. The Diabetes Educator, 33(1), 6978.

Alaofè, H., Asaolu, I., Ehiri, J., Moretz, H., Asuzu, C., Balogun, M., ... Ehiri, J. (2017). Community health workers in diabetes prevention and management in developing countries. Annals of Global Health, 83(3-4), 661-675.

Ali, M., Echouffo-Tcheugui, J., \& Williamson, D. (2012). How effective were lifestyle interventions in real-world settings that were modeled on the diabetes prevention program? Health Affairs, 31(1), $67-75$.

American Diabetes Association. (2017). Diabetes basics: type 2. http://www.diabetes.org/diabetesbasics/type-2/?loc $=$ db-slabnav

American Heart Association Cholesterol. (2018). http://www.heart.org/HEARTORG/Conditions/Cholesterol/Cholesterol_UCM_001089_SubHom ePage.jsp

Agarwal, G., Angeles, R. N., McDonough, B., McLeod, B., Marzanek, F., Pirrie, M., \& Dolovich, L. (2015). Development of a community health and wellness pilot in a subsidised seniors' apartment building in hamilton, ontario: Community health awareness program delivered by emergency medical services (CHAP-EMS). BMC Research Notes, 8.

Appalachian Regional Commission. (2017). The appalachian region. http://www.arc.gov/appalachian_region/TheAppalachianRegion.asp).

Arpey, N.C., Gaglioti, A.H., Rosenbaum, M.E. (2017). How socioeconomic status affects patient perceptions of health care: A qualitative study. Journal of Primary Care \& Community Health, 8 (3), 169-175

Baker, M.K., Simpson, K., Lloyd, B., Bauman, A.E., \& Singh, M.A. (2011). Behavioral strategies in diabetes prevention programs: A systematic review of randomized controlled trials. Diabetes Research and Clinical Practice, 91(1), 1-12.

Balagopal, P., Kamalamma, N., Patel, T.G., \& Misra, R. (2008). A community-based diabetes prevention and management education program in a rural village in india. Diabetes Care, 31(6), 1097-104. 
Balagopal, P., Kamalamma, N., Patel, T.G., \& Misra, R. (2012). A community-based participatory diabetes prevention and management intervention in rural india using community health workers. The Diabetes Educator, 38(6), 822-34.

Baek, R.N., Tanenbaum, M.L., Gonzalez, J.S. (2014). Diabetes burden and diabetes distress: The buffering effect of social support. Annals of Behavioral Medicine, 48(2), 145-55.

Barrera, M. (1986). Distinctions between social support concepts, measures, and models. American Journal of Community Psychology, 14(4), 413-445.

Bauer, W., Growick, B.M. (2003). Rehabilitation counseling in appalachian america. (Appalachian Rehabilitation). Journal of Rehabilitation, 69(3).

Beets, M., Cardinal, B., \& Alderman, B. (2010). Parental social support and the physical activity-related behaviors of youth: A review. Health Education \& Behavior, 37(5) 621-644.

Belalcazar, L.M., Reboussin, D.M., Haffner, S.M., Hoogeveen, R.C., Kriska, A.M., Schwenke ,D.C.,...Ballantyne, C.M. (2010). A 1-year lifestyle intervention for weight loss in individuals with type 2 diabetes reduces high c-reactive protein levels and identifies metabolic predictors of change: From the look ahead (action for health in diabetes) study. Diabetes Care, 33(11), 2297303.

Biedenweg, K., Meischke, H., Bohl, A., Hammerback, K., Williams, B., Poe, P., \& Phelan, E. (2014). Understanding older adults' motivators and barriers to participating in organized programs supporting exercise behaviors. The Journal of Primary Prevention, 35(1), 1-11.

Birch, D. (1998). Identifying sources of social support. Journal of School Health, 68(4), 159-61.

Bishop, J., Irby, M. B., Isom, S., Blackwell, C. S., Vitolins, M. Z., \& Skelton, J. A. (2013). Diabetes prevention, weight loss, and social support: program participants' perceived influence on the health behaviors of their social support system. Family \& Community Health, 36(2).

Black, S., Maitland, C., Hilbers, J., \& Orinuela, K. (2017). Diabetes literacy and informal social support: a qualitative study of patients at a diabetes centre. Journal of Clinical Nursing, 26, 248-257.

Brashers, D.E., Neidig, J.L., \& Goldsmith, D.J. (2004). Social support and the management of uncertainty for people living with hiv or aids. Health Communication, 16(3), 305-31.

Brinkmann, S., \& Kvale, S. (2015). Interviews: Learning the craft of qualitative research interviewing (3rd ed.). Thousand Oaks, CA: Sage.

Burke, V., Beilin, L.J., Cutt, H.E., Mansour, J., Mori, T.A. (2008). Moderators and mediators of behaviour change in a lifestyle program for treated hypertensives: A randomized controlled trial (ADAPT). Health Education Research, 23(4), 583-91.

Cantley-Falk, R. (2002). Hillbilly stereotype is no joke: Negative images of west virginia hurt growth, encourage young to leave, poll says. Huntington Herald Dispatch, April 30. 
Cargo, M., \& Mercer, S. (2008). The value and challenges of participatory research: Strengthening its practice. Annual Reviews in Public Health, 29, 325-350.

Caro-Bautista, J., Martín-Santos, F. J., Villa-Estrada, F., Morilla-Herrera, J. C., Cuevas-FernándezGallego, M., \& Morales-Asencio, J. M. (2015). Using qualitative methods in developing an instrument to identify barriers to self-care among persons with type 2 diabetes mellitus. Journal of Clinical Nursing, 24, 1024-1037.

Cavender, A., \& Beck, S. (1995). Generational change, folk medicine, and medical self-care in a rural appalachian community. Human Organization, 54(2), 129-129.

Centers for Disease Control and Prevention. National diabetes fact sheet, 2011. http://www.cdc.gov/diabetes/pubs/pdf/ndfs_2011.pdf[PDF - 2.66 MB] Accessed December 20, 2013.

Centers for Disease Control and Prevention. (2014). National diabetes statistics report: Estimates of diabetes and its burden in the united states, Atlanta, GA: US Department of Health and Human Services.

Centers for Disease Control and Prevention. (2015). Adult obesity facts, Atlanta, GA: US Department of Health and Human Services.

Centers for Disease Control and Prevention. Leading causes of death and numbers of deaths, by sex, race, and Hispanic origin: United States, 1980 and 2014 (Table 19). Health, United States, 2015. https://www.cdc.gov/nchs/data/hus/hus15.pdf\#019[PDF - 13.4 MB].

Centers for Disease Control and Prevention. (2017). Chronic disease prevention and health promotion, diabetes: Working to reverse the us epidemic at a glance 2016, Atlanta, GA: US Department of Health and Human Services.

Centers for Disease Control and Prevention. (2018). Behavioral risk factor surveillance system, Atlanta, GA: US Department of Health and Human Services.

Chan, R.S., Lok, K.Y., Sea, M.M., \& Woo, J. (2009). Clients' experiences of a community based lifestyle modification program: A qualitative study. International Journal of Environmental Research and Public Health, 6(10), 2608-22.

Cohen, S., Gottlieb, B.H., Underwood, L.G. (2000). Social relationships and health in social support measurement and intervention. A Guide for Health and Social Scientists eds S Cohen, LG Underwood \& BH Gottlieb, pp 3-25. Oxford: Oxford University Press.

Cohen, S., Wills, T.A. (1985). Stress, social support, and the buffering hypothesis. Psychological Bulletin, 98(2),310-357.

Coyne, C.A., Demain-Popescu, C., Friend, D. (2006). Social and cultural factors influencing health in southern west virginia: A qualitative study. Preventing Chronic Disease, 3(4), A124.

Cook, C., \& Baisden, D. (1986). Ancillary use of folk medicine by patients in primary care clinics in southern west virginia. Southern Medical Journal, 79. 
Creswell, J. W. (2013). Qualitative inquiry and research design: Choosing among five approaches. Third edition. Washington DC: Sage.

Diabetes Prevention Program Research Group. (2015). Long-term effects of lifestyle intervention or metformin on diabetes development and microvascular complications over 15-year follow-up: the diabetes prevention program outcomes study. The Lancet Diabetes \& Endocrinology, 3(11), 866-875.

Diabetes Prevention Program Research Group. (2002). Reduction in the incidence of type 2 diabetes with lifestyle intervention or metformin. New England Journal of Medicine, 346, 393-403.

Dankwa-Mullan, I., Rhee, K., Stoff, D., Pohlhaus, J., Sy, F., Stinson, N., \& Ruffin, J. (2010). Moving toward paradigm-shifting research in health disparities through translational, transformational, and transdisciplinary approaches. American Journal of Public Health, 100(19).

Davidov, D., Coffman, J., Dyer, A., Bias, T., Kristjansson, A., Mann, M.,...Abildso, C.(2018). Assessment and response to intimate partner violence in home visitation: A qualitative needs assessment with home visitors in a statewide program. Journal of Interpersonal Violence.

Davis-Smith, Y.M., Davis-Smith, M., Boltri J.M., et al. (2007). Implementing a diabetes prevention program in a rural african-american church. Journal of the National Medical Association, 99(4), 440-446.

Dougherty, D. \& Conway, P.H. (2008). The "3T's" road map to transform us health care: The "how" of high-quality care. Journal of the American Medical Association, 299(19), 2319-21.

Eddy, D.M., Schiessinger, L., Kahn, R. (2005). Clinical outcomes and cost-effectiveness of strategies for managing people at high risk for diabetes. Annals of Internal Medicine, 143(4), 251-W-68.

Eriksson, K. F., \& Lindgärde, F. (1991). Prevention of type 2 (non-insulin-dependent) diabetes mellitus by diet and physical exercise The 6-year malmö feasibility study. Diabetologia, 34(12), 891-898.

Faridi, Z., Shuval, K., Njike, V.Y., et al. (2010). Partners reducing effects of diabetes (PREDICT): A diabetes prevention physical activity and dietary intervention through african-american churches. Health Education Research, 25(2), 306-315.

Fetterman, D.M., Wandersman, A. (2005). Empowerment evaluation principles in practice. New York: Guilford.

Flicker, S., Travers, R., Guta, A., McDonald, S., Meagher, A. (2007). Ethical dilemmas in communitybased participatory research: recommendations for institutional review boards. Journal of Urban Health, 84(4),478-93.

Forsythe, L. P., Alfano, C. M., Kent, E. E., Weaver, K. E., Bellizzi, K., Arora, N., ... Rowland, J. H. (2014). Social Support, self-efficacy for decision making, and follow-up care use in long-term cancer survivors. Psycho-Oncology, 23(7), 788-796.

Fowler, J.L., Follick, M.J., Abrams, D.B., Rickard-Figueroa, K. (1985). Participant characteristics as predictors of attrition in worksite weight loss. Addictive Behaviors, 10, 445-448. 
French, S. A., Jeffery, R. W., Story, M., \& Neumark-Sztainer, D. (1998). Perceived barriers to and incentives for participation in a weight-loss program among low-income women in wic. Journal of the American Dietetic Association, 98(1),79-81.

Gallant, M.P. (2003). The influence of social support on chronic illness self-management: a review and directions for research. Health Education Behavior, 30(2),170-95.

Glanz, K., Rimer, B., \& Viswanath, K. (2008). Health behavior and health education : Theory, research, and practice(4th ed. ed.). San Francisco, CA: Jossey-Bass.

Glasgow, R.E., McKay, H.G., Piette, J.D., Reynolds, K.D. (2001). The RE-AIM framework for evaluating interventions: What can it tell us about approaches to chronic illness management? Patient Education and Counseling, 44(2), 119-27.

Glasgow, R. E., Vogt, T. M., \& Boles, S. M. (September 01, 1999). Evaluating the public health impact of health promotion interventions: The RE-AIM framework. American Journal of Public Health, 89(9), 1322-7.

Goh, Y., Bogart, L., Sipple-Asher, B., Uyeda, K. Hawes-Dawson, J. Olarita-Dhungana, J...Schuster, M. (2008). Using community-based participatory research to identify potential interventions to overcome barriers to adolescents' healthy eating and physical activity. Journal of Behavioral Medicine, 32, 491-502.

Gonzalez, J.S., Tanenbaum, M.L., Commissariat, P.V. (2016). Psychosocial factors in medication adherence and diabetes self-management: Implications for research and practice. American Psychology, 71(7), 539-551.

Green, L. (2008). Making research relevant: If it is an evidence-based practice, where's the practicebased evidence? Family Practice, 25(S1), 24.

Hag Hamed, D. (2017). The influence of fatalistic beliefs on health beliefs among diabetics in Khartoum, Sudan: a comparison between Coptic Christians and Sunni Muslims. Global Health Promotion.

Halverson, J. A., Ma, L., Harner, E.J., Appalachian Regional Commission, West Virginia University Office for Social Environment and Health Research. (2004). An analysis of disparities in health status and access to health care in the Appalachian Region.

Hansen, E., Landstad, B., Hellzén, O., \& Svebak, S. (2011). Motivation for lifestyle changes to improve health in people with impaired glucose tolerance. Scandinavian Journal of Caring Sciences, 25(3), 484-490.

Harry, B., Sturges, K. M., \& Klingner, J. K. (2005) Mapping the process: An exemplar of process and challenge in grounded theory analysis. Educational Researcher, 34(2), 3-13. 
Heard, E., Whitfield, K.E., Edwards, C.L., Bruce, M., Beech, B.M. (2013). Mediating effects of social support on the relationship among perceived stress, depression, and hypertension in african americans. Journal of the National Medical Association, 103(2),116-22.

Heisler, M. (2007). Abstract overview of peer support models to improve diabetes self-management and clinical outcomes. Diabetes Spectrum, 20(4), 214-221.

Helton, L. (1995). Intervention with Appalachians: strategies for a culturally specific practice. Journal of Cultural Diversity, 2(1), 20-6.

Helton, L., \& Keller, S. (2010). Appalachian women: A study of resiliency assets and cultural values. Journal of Social Service Research, 36(2), 151-161.

Hernan, W.H., Brandle, M., Zhang, P. Williamson, D.F., Matulik, M.J., Ratner, R.E....Diabetes Prevention Program Research Group. (2003). Costs associated with the primary prevention of type 2 diabetes mellitus in the diabetes prevention program. Diabetes Care, 26(1), 36-47.

Horn, K., McCracken, L., Dino, G., \& Brayboy, M. (2006). Application of community-based participatory research principles to the development of a smoking cessation program for american indian teens. Journal of Health Education and Behavior.

Hunt, C. W., Grant, J. S., \& Pritchard, D. A. (April 01, 2012). An empirical study of self-efficacy and social support in diabetes self-management. Home Healthcare Nurse, 30 (4), 255-262.

Hupcey, J. (1998). Clarifying the social support theory-research linkage. Journal of Advanced Nursing, 27(6), 1231-1241.

Hwang, K. O., Etchegaray, J. M., Sciamanna, C. N., Bernstam, E. V., \& Thomas, E. J. (2014). Structural social support predicts functional social support in an online weight loss programme. Health Expectations: An international journal of public participation in health care and health Policy, 17(3), 345-52.

IBM Corp. Released 2017. IBM SPSS Statistics for Windows, Version 25.0. Armonk, NY: IBM Corp.

Ingels, J.S., Misra, R., Stewart, J., Lucke-Wold, B., Shawley-Brzoska, S. (2017). The effect of adherence to dietary tracking on weight loss: Using hlm to model weight loss over time. Journal of Diabetes Research.

Islam, S., Purnat, T., Phuong, N., Mwingira, U., Schacht, K., \& Fröschl, G. (2014). Non-communicable diseases (NCDs) in developing countries: A symposium report. Globalization and Health, 10(1).

Jackson, L. (2009). Translating the diabetes prevention program into practice: A review of community interventions. The Diabetes Educator, 35(2), 309-320.

Jeffery, R.W., Thompson, P.D.,Wing, R.R. (1978) Effects on weight reduction of strong monetary contracts for calorie restriction or weight loss. Behavioral Research Theories, 16(5), 363-9. 
Johnson, L., \& Melton, S. (2016). Perceived benefits and barriers to the diabetes prevention program. PLAID: People living with and inspired by diabetes, 2(1).

Jones, L., Wells, K. (2007). Strategies for academic and clinician engagement in community participatory partnered research. Journal of American Medical Association, 297, 407-10.

Katula, J.A., Vitolins, M.Z., Rosenberger, E.L., et al. (2011). One-year results of a community-based translation of the diabetes prevention program: Healthy-living partnerships to prevent diabetes (HELP PD) project. Diabetes Care, 34(7), 1451-1457.

Kamphuis, C.B., Giskes, K., De Bruijn, G.J., Wendel-Vos, W., Brug, J., \& Van Lenthe, F.J. (2006). Environmental determinants of fruit and vegetable consumption among adults: A systematic review. British Journal of Nutrition, 96(4), 620-35.

Kegler, M. C. \& Alcantara, I. \& Veluswamy, J. K. \& Haardörfer, R., Hotz, J. A., \& Glanz, K. (2012). Results from an intervention to improve rural home food and physical activity environments. Progress in Community Health Partnerships: Research, Education, and Action, 6(3), 265-277. The Johns Hopkins University Press.

Kemmis, S., McTaggart, R. (2000). Participatory action research. In handbook of qualitative research, ed. NK Denzin, YS Lincoln, pp. 567-605. Thousand Oaks, CA: Sage

Kessler, R. C., McLeod, J. D. (1984). Sex differences in vulnerability to undesirable life events. American Sociological Review, 49, 620-631.

Kitzman, H., Dodgen, L., Mamun, A., Slater, J.L., King, G., Slater, D.,...DeHaven, M. (2017). Community-based participatory research to design a faith-enhanced diabetes prevention program: The better me within randomized trial. Contemporary Clinical Trials, 62, 77-90.

Kramer, M., Miller, R., \& Siminerio, L. (2014). Evaluation of a community diabetes prevention program delivered by diabetes educators in the united states: One-year follow up. Diabetes Research and Clinical Practice, 106(3), 52.

Kishore, J., Kohli, C., Gupta, N., Kumar, N., Gupta, N, \& Ray, P.C. (2013). Initial psychological reaction and social support in patients of type 2 diabetes mellitus in Delhi. International Journal of Medicine and Public Health, 3(4), 244-249.

Kishore, G., Nagaraj, P., \& Ravouru, N. (2013). Health-related quality of life in diabetes. Value in Health, 16(3), 197.

Knowler, W.C., Fowler, S.E., Hamman, R.F., Christophi, C.A., Hoffman, H.J. Brenneman, A.T.....Diabetes Prevention Program Research Group. (2009). 10-year follow-up of diabetes incidence and weight loss in the diabetes prevention program outcomes study. Lancet. 374(9702), 1677.

Knox, S.S., Basu, S., \& Remick, S. (2014). A systems approach to cancer health disparities in appalachia. Austin Journal of Public Health Epidemiology, 11, 1004. 
Korkiakangas, E. E., Alahuhta, M. A., Husman, P. M., Keinänen-Kiukaanniemi, S., Taanila, A. M., \& Laitinen, J. H. (2011). Motivators and barriers to exercise among adults with a high risk of type 2 diabetes-A qualitative study. Scandinavian Journal of Caring Sciences, 25(1), 62-9.

Kozica, S., Gibson-Helm, M., Teede, H., \& Moran, L. (2013). Assessing self-efficacy and self-help methods in women with and without polycystic ovary syndrome. Behavioral Medicine, 39(3), 90-96.

Kramer, F.M., Jeffery, R.W., Snell, M.K., Forster, J.L. (1986). Maintenance of successful weight loss over 1 year: effects of financial contracts for weight maintenance or participation in skills training. Behavior Therapy. 17(3), 295-301

Kramer, M.K., McWilliams, J.R., Chen, H.-Y., Siminerio, L.M. (2011). A community-based diabetes prevention program: Evaluation of the group lifestyle balance program delivered by diabetes educators. The Diabetes Educator. 37(5), 659-668.

Lakey, B., Cronin, A. (2008). Low social support and major depression: Research, theory, and methodological issues. Risk factors for depression. Academic Press. pp. 385-408.

Lambert, L., Raidl, M., Safaii, S. M., Conner, C. R., Geary, E. J. M., \& Ault, S. B. (2005). Perceived benefits and barriers related to postpartum weight loss of overweight/obese postpartum wic participants. Topics in Clinical Nutrition, 20(1), 16-27.

Lanese, B., Dey, A., Srivastava, P., \& Figler, R. (2011). Introducing the health coach at a primary care practice: Impact on quality and cost (Part 1). Hospital Topics, 89(1), 16-16.

Langford, C.P., Bowsher, J., Maloney, J.P., Lillis, P.P. (1997). Social support: A conceptual analysis. Journal of Advanced Nursing, 25(1), 95-100.

Levin, J.S. (1984). The role of the black church in community medicine. Journal of National Medical Association, 76(5), 477-483.

Lewis, R., \& Billings, D. (1997). Appalachian culture and economic development: A retrospective view on the theory and literature. Journal of Appalachian Studies, 3(1), 3-42.

Lidegaard, L., Schwennesen, N., Willaing, I., \& Færch, K. (2016). Barriers to and motivators for physical activity among people with type 2 diabetes: Patients' perspectives. Diabetic Medicine, 33(12), 1677-1685.

Lincoln, Y.S., Guba, E.G. (1985). Naturalistic Inquiry. Newbury Park, CA: Sage Publications.

Lindström, J., Louheranta, A., Mannelin, M., Rastas, M., Salminen, V., Eriksson, J.,...Tuomilehto, J. (2003). The finnish diabetes prevention study (DPS) lifestyle intervention and 3-year results on diet and physical activity. Diabetes Ccare, 26(12), 3230-3236.

Lucke-Wold, B., Shawley, S., Ingels, J.S., Stewart, J., Misra, R. (2016). A critical examination of the use of trained health coaches to decrease the metabolic syndrome for participants of a 
community-based diabetes prevention and management program. Journal of Health Communication, 1(4).

Ma, L., Li, Y., Wang, J., Zhu, H., Yang, W., Cao, R.,...Feng, M. (2015). Quality of life is related to social support in elderly osteoporosis patients in a chinese population. PLOS One, 10(6), e0127849.

Marquez, B., Anderson, A., Wing, R.R., West, D.S., Newton, R.L., Meacham, M.,.. Look AHEAD Research Group. (2016). The relationship of social support with treatment adherence and weight loss in Latinos with type 2 diabetes. Obesity, 24(3), 568-75.

Mayo Clinic. (2018). Diseases \& Conditions. Cholesterol. https://www.mayoclinic.org/diseasesconditions/high-blood-cholesterol/symptoms-causes/syc-20350800 2018

McEvoy, P.M., \& Nathan, P. (2007). Perceived costs and benefits of behavioral change: Reconsidering the value of ambivalence for psychotherapy outcomes. Journal of Clinical Psychology, 63(12), 1217-29.

McQuiston, C., Flaskerud, J.H.. (2003). If they don't ask about condoms, I just tell them: a descriptive case study of latino health advisers' helping activities. Health Education Behavior, 30, 79-96.

Miech, R., Kim, J., McConnell, C., \& Hamman, R. (2009). A growing disparity in diabetes-related mortality : U.S. trends, 1989-2005. American Journal of Preventive Medicine, 36(2), 126-132.

Minkler, M. (2005). Community-based research partnerships: Challenges and opportunities. Journal of Urban Health, 822, ii3-ii12.

Misra, R. Sambamoorthi, U. (2017). Diabetes disparities in west virginia: 2010-2014. American Public Health Association. https://apha.confex.com/apha/2017/meetingapp.cgi/Paper/391189

Mitchell, C., Stuart, R.B. (1984). Effect of self-efficacy on dropout from obesity treatment. Journal of Consulting and Clinical Psychology, 52, 1100-1101.

Mollaoğlu, M., \& Beyazıt, E. (2009). Influence of diabetic education on patient metabolic control. Applied Nursing Research, 22(3), 183-190.

Murray, C.J.L., \& Lopez, A.D. (1996) The global burden of disease: a comprehensive assessment of mortality and disability from diseases, injuries, and risk factors in 1990 and projected to 2020. Cambridge, Harvard School of Public Health on behalf of the World Health Organization and the World Bank, 1996 (Global Burden of Disease and Injury Series, Vol. 1).

National Council on Aging. 2018. Chronic disease-management map of partners \& programs.

https://www.ncoa.org/map/ncoa-map/

National Institute of Diabetes and Digestive and Kidney Diseases. 2018. Diabetes. Diabetes Overview. What is Diabetes. https://www.niddk.nih.gov/health-information/diabetes/overview/what-isdiabetes 
Neamah, H.H., Sebert Kuhlmann, A.K., \& Tabak, R.G. (2016). Effectiveness of program modification strategies of the diabetes prevention program: A systematic review. The Diabetes Educator, 42(2), 153-65.

Niedermann, K., Hammond, A., Forster, A., \& De, B. R. (September 01, 2010). Perceived benefits and barriers to joint protection among people with rheumatoid arthritis and occupational therapists. A mixed methods study. Musculoskeletal Care, 8(3), 143-156.

Ogden, C.L., Carroll, M.D., Fryar, C.D., \& Flegal, K.M. (2015) Prevalence of obesity among adults and youth: United States, 2011-2014. NCHS Data Brief, 219, 1-8.

Osborn, C. Y., Bains, S. S., \& Egede, L. E. (January 01, 2010). Health literacy, diabetes self-care, and glycemic control in adults with type 2 diabetes. Diabetes Technology \& Therapeutics, 12(11), 913-9.

Pan, X. R., Li, G. W., Hu, Y. H., Wang, J. X., Yang, W. Y., An, Z. X.,...Jiang, X. G. (1997). Effects of diet and exercise in preventing NIDDM in people with impaired glucose tolerance: The da qing igt and diabetes study. Diabetes Care, 20(4), 537-544.

Paskett, E.D., Fisher, J.L., Lengerich, E.J., Schoenberg, N.E., Kennedy, S.K., Conn, M.E.,...Dignan, M. (2011). Disparities in underserved white populations: The case of cancer-related disparities in Appalachia. Oncologist. 16(8), 1072-81.

Pender, N.J., Murdaugh, C.L., \& Parsons, M.A. (2002). Health-promotion in nursing practice. USA: Prentice Hall, 4(60).

Piette, J.D., Aikens, J.E., Trivedi, R., Parrish, D., Standiford, C., Marinec, N.S.,...Bernstein, S.J. (2013). Depression self-management assistance using automated telephonic assessments and social support. The American Journal of Managed Care, 19(11), 892-900

Pullen, C., Walker, S.N., Fiandt, K. (2001). Determinants of health-promoting lifestyle behaviors in rural older women. Family \& Community Health, 24(2), 49-72.

Rad, G.S., Bakht, L.A., Feizi, A., Mohebi, S. (2013). Importance of social support in diabetes care. Journal of Education and Health Promotion, 2(62).

Rees, C.A., Karter, A., Young, B.A. (2010). Race/ethnicity, social support, and associations with Diabetes Self-Care and Clinical Outcomes in NHANES. Diabetes Educator, 36(3), 435-445.

Reblin, M., Uchino, B.N. (2008). Social and emotional support and its implication for health. Current Opinion in Psychiatry, 21(2), 201-5

Resnicow, K. et al. (2004) Body and soul. A dietary intervention conducted through African American churches. American Journal of Preventive Medicine, 27(2), 97-105.

Rosenson RS, et al. HDL-cholesterol: Clinical aspects of abnormal values. http://www.uptodate.com/home. Accessed June 1, 2016. 
Reyes, N.R., Oliver, T.L., Klotz, A.A., Lagrotte, C.A., Vander Veur, S.S., Virus, A.,...Foster, G.D. (2012). Similarities and differences between weight loss maintainers and regainers: A qualitative analysis. Journal of the Academy of Nutrition and Dietetics, 112(4), 499-505.

Rosland, A.M., Kieffer, E., Israel, B., Cofield, M., Palmisano, G., Sinco, B.,...Heisler, M.(2008). When is social support important? The association of family support and professional support with specific diabetes self-management behaviors. Journal of General Internal Medicine, 23(12), 1992-9.

Rosland, A.M., Piette, J.D., Lyles, C.R., Parker, M.M., Moffet, H.H., Adler, N.E., ,...Karter A.J. (2014). Social support and lifestyle vs. medical diabetes self-management in the diabetes study of northern california (DISTANCE). Annals of Behavioral Medicine: A Publication of the Society of Behavioral Medicine, 48(3), 438-47.

Rovner, A. J., Mehta, S. N., Haynie, D. L., Robinson, E. M., Pound, H. J., Butler, D. A., ... Nansel, T. R. (2010). Perceived benefits, barriers, and strategies of family meals among children with type 1 diabetes and their parents: Focus group findings. Journal of the American Dietetic Association, 110(9), 1302-1306.

Royse, D., Dignan, M. (2011). Fatalism and cancer screening in appalachian kentucky. Family Community Health, 2, 126-33

Sallis, J.F., Grossman, R.M., Pinski, R.B., Patterson, T.L., Nader, P.R. (1987). The development of scales to measure social support for diet and exercise behaviors. Preventive Medicine, 16, 825836.

Sallis, J.; Owen, N. Physical activity and behavioral medicine. Thousand Oaks, CA: Sage Publications; 1999.

Sandelowski, M., \& Barroso, J. (2007) Handbook for synthesizing qualitative research. New York: Springer.

Sarason, B.R., Sarason, I.G. (2009). Social support: Mapping the construct. Journal of Social and Personal Relationships, 26(1), 113-120

Self-Management Resource Center. Programs. 2018. https://www.selfmanagementresource.com/programs/

Shah, M.K., Kieffer, E.C., Choi, H., Schumann, C., Heisler, M. (2015). Mediators and moderators of the effectiveness of a community health worker intervention that improved dietary outcomes in pregnant latino women. Health Education Behavior, 42(5), 593-603.

Shaikh, A.R., Yaroch, A.L., Nebeling, L., Yeh, M.C., Resnicow, K. (2008). Psychosocial predictors of fruit and vegetable consumption in adults a review of the literature. American Journal of Preventive Medicine, 34(6), 535-543.

Shandera-Ochsner, A.L., Han, D.Y., Rose, D., Aroor, S.R., Schmitt, F., Bellamy, L.M., \& Dobbs, M.R. (2014). Comparing the trends of elevated blood pressure in appalachian and non-Appalachian regions. Journal of Clinical Hypertension (greenwich, Conn.), 16(10), 713-5. 
Sharifirad, G., Azadbakht, L., Feizi, A., Kargar, M., \& Mohebi, S. (2013). Structural role of perceived benefits and barriers to self-care in patients with diabetes. Journal of Education and Health Promotion, 2(1), 37-37.

Shawley-Brzoska, S., Misra, R. (2018). Perceived benefits and barriers of a community-based diabetes prevention and management program. Journal of Clinical Medicine, 7(3), 58.

Shenton, A. (2004). Strategies for ensuring trustworthiness in qualitative research projects. Education for Information, 22, 63-75.

Sherbourne, C.D., Stewart, A.L. (1991). The MOS social support survey. Social Sciences \& Medicine Journal, 32(6), 705-714.

Shier, G., Ginsburg, M., Howell, J., Volland, P., \& Golden, R. (2013). Strong social support services, such as transportation and help for caregivers, can lead to lower health care use and costs. Health Affairs, 32(3), 544-51.

Simon, J.G., De Boer, J.B., Bosma, H., Mackenbach, J.P. How is your health in general? A qualitative study on self-assessed health. European Journal of Public Health, 15(2),200-8.

Smith, K.L., Straker, L.M., McManus, A., Fenner, A.A. (2014). Barriers and enablers for participation in healthy lifestyle programs by adolescents who are overweight: a qualitative study of the opinions of adolescents, their parents and community stakeholders. BMC Pediatrics, 14,(53).

Stevens, E.S. (1992). Reciprocity in social support: An advantage for the aging family. The Journal of Contemporary Human Social support, hardiness and psychological well-being in women with arthritis. Image: Journal of Nursing Scholarship Services, 533-54.

Strain, C.B. (2002). Folk Medicine in Southern Appalachian Fiction. East Tennessee State University

Straus, S., Tetroe, J., \& Graham, I. (2013). Knowledge translation in health care: Moving from evidence to practice (2nd ed. ed.). Chichester, West Sussex: John Wiley \& Sons.

Tabish, S. A. (2007). Is diabetes becoming the biggest epidemic of the twenty-first century? International Journal of Health Sciences, 1(2).

Taborsky B., \& Oliveria, R.F. (2012). Social competence: An evolutionary approach. Trends in Ecology \& Evolution, 27(12), 679-88.

Teixeira, P.J., Going, S.B., Houtkooper, L.B., Cussler, E.C., Metcalfe, L.L., Blew, R.M.,..Lohman, T.G. (2004). Pretreatment predictors of attrition and successful weight management in women. International Journal of Obesity, 28, 1124-1133. 
The Diabetes Prevention Program Research Group. (1999). The diabetes prevention program: Design and methods for a clinical trial in the prevention of type 2 diabetes. Diabetes Care. 22(4), 623634.

The Emerging Risk Factors Collaboration. (2010). Diabetes mellitus, fasting blood glucose concentration, and risk of vascular disease: A collaborative meta-analysis of 102 prospective studies. The Lancet. 37(9733), 2215-2222.

Thomson, R.L., Buckley, J.D., Brinkworth, G.D. (2016). Perceived exercise barriers are reduced and benefits are improved with lifestyle modification in overweight and obese women with polycystic ovary syndrome: A randomised controlled trial. BMC Womens Health, 16(14).

Thorne, S. (1998). Ethical and representational issues in qualitative secondary analysis. Qualitative Health Research, 8(4),547-55.

Toobert, D. J., Hampson, S. E., \& Glasgow, R. E. (January 01, 2000). The summary of diabetes self-care activities measure: results from 7 studies and a revised scale. Diabetes Care, 23(7), 943-50.

George Towers (2007) West virginia's lost youth: Appalachian stereotypes and residential preferences, Journal of Geography, 104(2),74-84.

Tripp-Reimer, T., \& Doebbeling, B. (2004). Qualitative perspectives in translational research. Worldviews on Evidence-Based Nursing, 1, 65-72.

Trost, S.G., Owen, N., Bauman, A.E., Sallis, J.F., Brown, W. (2002). Correlates of adults' participation in physical activity: Review and update. Medical and Sciences in Sports Exercise, 34(12), 19962001.

Turner, A., Phillips, L. Hambridgem, J.A. Baker, A.L. Bowman, J., Colyvas, K. (2010). Clinical outcomes associated with depression, anxiety and social support among cardiac rehabilitation attendees. The Australian and New Zealand Journal of Psychiatry, 44(7), 658-66.

Uchino, B., Cacioppo, J., \& Kiecolt-Glaser, J. (1996). The relationship between social support and physiological processes: A review with emphasis on underlying mechanisms and implications for health. Psychological Bulletin, 119(3), 488-488.

Uchino, B. (2004). Social Support and Physical Health: Understanding the Health Consequences of Relationships. New Haven, CT: Yale University Press.

Uchino, B. (2009). Understanding the links between social support and physical health: A life-Span perspective with emphasis on the separability of perceived and received support. Perspectives on Psychological Science: A Journal of the Association for Psychological Science,4(3), 236-55.

Uchino, B., Bowen, K., Carlisle, M., \& Birmingham, W. (2012). Psychological pathways linking social support to health outcomes: A visit with the "ghosts" of research past, present, and future. Social Science \& Medicine, 74(7), 949-957.

U.S. Census Bureau (2015). Income, Poverty and Health Insurance Coverage in the United States: 2014. Retrieved from https://www.census.gov/newsroom/press-releases/2015/cb15-157.html. 
Vadheim, L.M., Brewer, K.A., Kassner, D.R., Vanderwood, K.K., Hall, T.O., Butcher, M.K.,....Harwell, T.S. (2010). Effectiveness of a lifestyle intervention program among persons at high risk for cardiovascular disease and diabetes in a rural community. The Journal of Rural Health, 26(3),266-272.

Van Dam, H., Van der Horst, F., Knoops, L., Ryckman, R., Crebolder, H., \& Van den Borne, B. (2005). Social support in diabetes: A systematic review of controlled intervention studies. Patient Education and Counseling, 59(1), 1-12.

Van Den Arend, I., Stolk, R., Rutten, G., \& Schrijvers, G. (2000). Education integrated into structured general practice care for type 2 diabetic patients results in sustained improvement of disease knowledge and self-care. Diabetic Medicine, 17(3), 190-197.

Vangelisti, A. (2009). Challenges in conceptualizing social support. Journal of Social and Personal Relationships, 26(1), 39-51.

Venditti, E. M., Wylie-Rosett, J., Delahanty, L. M., Mele, L., Hoskin, M. A., \& Edelstein, S. L. (2014). Short and long-term lifestyle coaching approaches used to address diverse participant barriers to weight loss and physical activity adherence. The International Journal of Behavioral Nutrition and Physical Activity, 11.

Verheijden, M.W., Bakx, J.C., Van Weel, C., Koelen, M.A., Van Staveren, W.A. (2005). Role of social support in lifestyle-focused weight management interventions. European Journal of Clinical Nutrition, 59, 179-86.

Wallerstein, N., \& Duran, B. (2010). Community-based participatory research contributions to intervention research: The intersection of science and practice to improve health equity. American Journal of Public Health, 100(Suppl 1), S40-S46.

Wallhagen, M.I., Lacson, M. (1999). Perceived control and psychosocial/physiological functioning in african american elders with type 2 diabetes. The Diabetes Educator, 25(4), 568-75.

Ward, B.W., Schiller, J.S., Goodman, R.A. (2014). Multiple chronic conditions among U.S. adults: a 2012 update. Preventing Chronic Disease, 11, 130389.

Welch, W. (2011). Self-control, fatalism, and health in appalachia. Journal of Appalachian Studies, $17(1 \& 2), 108-122$.

West Virginia Department of Health and Human Resources (2012). HSC statistical brief diabetes and health equity in west virginia: A review 2012.

West Virginia Health Statistics Center. (2018). WV health statistic center data.

Wewers, M.E., Katz, M., Paskett, E.D., Fickle, D. (2006). Risky behaviors among ohio appalachian adults. Preventing Chronic Disease, 3(4), A127.

Wiebe, D.J., Helgeson, V., Berg, C.A. (2016). The social context of managing diabetes across the life span. American Psychologist, 71(7), $526-538$

Wills, T.A. (1991). Social support and interpersonal relationships. Prosocial Behavior, Review of Personality and Social Psychology, 12, 265-289. 
Wing, R.R., Tate, D.F., Gorin, A.A., Raynor, H.A., \& Fava, J.L. (2006). A self-regulation program for maintenance of weight loss. The New England Journal of Medicine, 355(15), 1563-71.

World Health Organization (2018). Global Health Observatory (GHO) data. NCD mortality and morbidity. http://www.who.int/gho/ncd/mortality_morbidity/en/

Yass-Reed, E.M., Barry, N.J., Dacey,C.M. (1993). Examination of pretreatment predictors of attrition in a VLCD and behavior therapy weight-loss program. Addictive Behaviors, 18(43). 